\title{
Dynamics for Variable Length Multisection Continuum Arms
}

\author{
Isuru S. Godage*, Gustavo A. Medrano-Cerda ${ }^{\ddagger}$, David T. Branson†, Emanuele Guglielmino ${ }^{\ddagger}$, \\ and Darwin G. Caldwell ${ }^{\ddagger}$
}

\begin{abstract}
Variable length multisection continuum arms are a class of continuum robotic manipulators that generate motion by structural mechanical deformation. Unlike most continuum robots, the sections of these arms do not have (central) supporting flexible backbone, and are actuated by multiple variable length actuators. Because of the constraining nature of actuators, the continuum sections can bend and/or elongate (compress) depending on the elongation/contraction characteristics of the actuators being used. Continuum arms have a number of distinctive differences with respect to traditional rigid arms namely: smooth bending, high inherent compliance, and adaptive whole arm grasping. However, due to numerical instability and the complexity of curve parametric models, there are no spatial dynamic models for multisection continuum arms. This paper introduces novel spatial dynamics and applies these to variable length multisection continuum arms with any number of sections. An efficient recursive computational scheme for deriving the equations of motion is presented. This is applied in a general form based on structurally accurate and numerically well posed modal kinematics that assumes circular arc deformation of continuum sections without torsion. It is shown that the proposed modal dynamics are highly scalable, producing efficient and accurate numerical results. The spatial dynamic simulation results are experimentally validated using a pneumatic muscle actuated multisection prototype continuum arm. For the first time this enables investigation of spatial dynamic effects in this class of continuum arms.
\end{abstract}

Keywords: continuum arms, modal shape functions, spatial dynamics, recursive computations

\section{Introduction}

Due to their inherent flexibility and compliance, soft continuum robots inspired by tongues (Takanobu et al., 2004), elephant trunks (Cieslak and Morecki, 1999; Hannan and Walker, 2003; Wolf et al., 2003), octopus arms (Cianchetti et al., 2011; Grissom et al., 2006), and other cephalopod appendages (McMahan et al., 2004), can elongate, contract, and bend at any point along their structure (Trivedi et al., 2008b). This allows them to, access highly confined spaces and follow complex trajectories. They have some unique and highly distinctive features when compared with rigid bodied robots: such as smooth bending, inherent compliance (Rucker and Webster, 2011a), reduced weight, and increased fault tolerance (Godage et al., 2011b). Continuum arms also have excellent potential for adaptive whole arm grasping (McMahan and Walker, 2008; Li and Xiao, 2011), obstacle avoidance (Godage et al., 2012a), navigation in highly unstructured, narrow and obstructed environments (Xiao and Vatcha, 2010), minimally invasive surgery (Lock et al., 2010; Lyons et al., 2009; Penning et al., 2011; Simaan et al., 2004; Rucker and Webster, 2011b), inspection tasks (Mehling et al., 2006), and human friendly interaction (Walker et al., 2005). In light of these advantages, continuum and soft robotics has become an area of major importance in bio-inspired robotics.

Figure 1 shows two, highly compliant, state of the art variable length multisection continuum arms that are powered by pneumatic muscle actuators (PMA). In this paper, a continuum "section" is identified as a unit that is capable of producing independent bending deformation whereas "segments" are a subset of a continuum section, i.e., multiple segments make up a section as shown in Figure 1b. Due to the particular constraining nature of variable length actuator arrangement, a continuum section deforms

\footnotetext{
*Isuru S. Godage (corresponding author) is with the Department of Electrical and Computer Engineering, Clemson University, Clemson SC 29634, USA. igodage@g.clemson.edu.

${ }^{\dagger}$ David T. Branson is with the Mechanical, Materials and Manufacturing Department, University of Nottingham, NG7 2RD, UK. david.branson@nottingham.ac.uk.

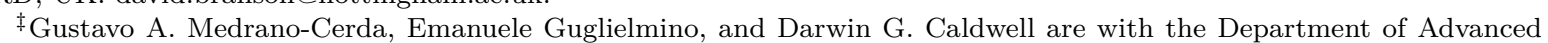
Robotics, Istituto Italiano di Tecnologia, Via Morego 30, 16163 Genoa, Italy. \{gustavo.cerda; emanuele.guglielmino; darwin.caldwell\}@iit.it.
} 


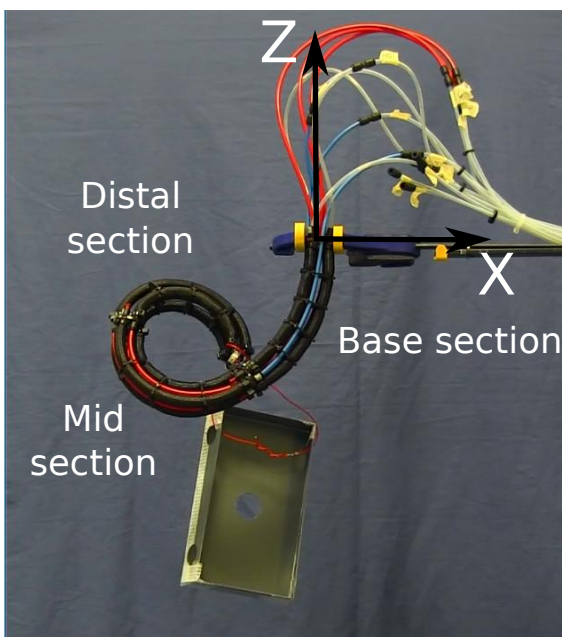

(a)

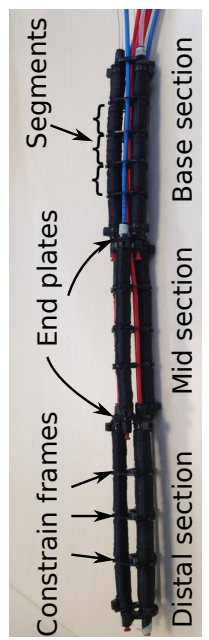

(b)

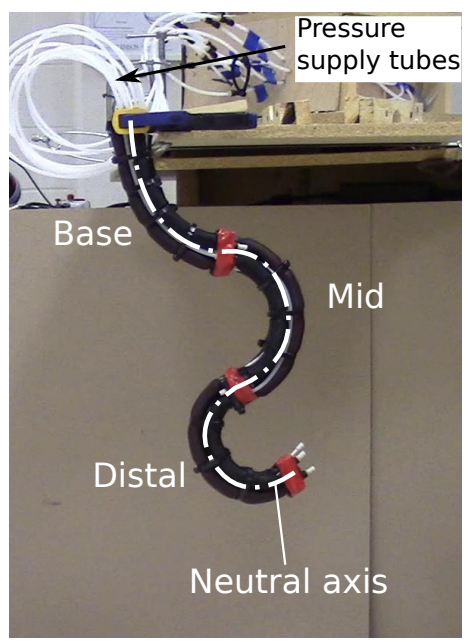

(c)

Figure 1: Pneumatic muscle actuated, three-section variable length continuum robotic arm prototypes. (a) The continuum arm developed at the Italian Institute of Technology (IIT) manipulating an object. Note the smooth circular arc shape bending of variable length continuum sections, (b) the prototype of Figure 1a is at rest without actuation. Section and segment terms are also indicated as referred to in this paper, (c) The continuum arm developed at Clemson University demonstrating multisection bending. Individual continuum sections and neutral axis are annotated for clarity.

in a circular arc (without torsion) where straight section poses are modeled as circular arcs of zero curvature. PMA's are particularly suitable for implementing variable length multisection continuum arms owing to their low cost, high flexibility and compliance, excellent length change ratio, and high power to weight ratio (Caldwell et al., 1995). Similar pneumatically actuated multisection continuum arms include OctArm (McMahan et al., 2006; Neppalli et al., 2007) and FESTO bionic handling assistant (Grzesiak et al., 2011; Mahl et al., 2014) among others. Multisection continuum arms are constructed by stacking at least two continuum sections where, each is actuated by multiple variable length actuators. These continuum sections are kinematically independent (bending of one section does not affect the others), generate motion by elastic deformation (that results in a length change along the neutral axis hence the term variable length), and not backbone supported (actuators provide the structural strength). These unique features therefore differentiate this class of continuum arms from other continuum arm implementations that employ: fixed-length flexible backbones (Gravagne and Walker, 2000; Rucker and Webster, 2011b), tendon/rod actuation (Camarillo et al., 2009; Xu and Simaan, 2010; Goldman et al., 2011), tendon-pneumatic hybrid actuation (Immega et al., 1995; McMahan et al., 2005), and concentric tubes (Webster et al., 2009; Dupont et al., 2010).

Lumped or segmented models for continuum arms represent the natural transition from the traditional, rigid multijointed robot dynamic modeling approaches. When used for relatively few rigid segments, lumped models avoid the complex expressions intrinsic in continuum arms to yield efficient results (Giri and Walker, 2011; Khalil et al., 2007). However, to accurately resemble the smooth bending of continuum arms many segments are required (Zheng et al., 2012), significantly increasing the overall degrees of freedom (DoF) in contrast to actual number of controlled joint variables.

Cosserat rod theory has been successfully applied to solve for statics/dynamics of flexible backbone supported, tendon actuated, and concentric type continuum arms. Jones et al. (2009) proposed three-dimensional statics for gravity loaded, unactuated flexible rods, while Rucker and Webster (2011b) generalized the approach for backbone supported tendon actuated continuum arms with general tendon routing. A similar approach was applied to multibending soft robot by Renda et al. (2014). Flexible manipulator dynamics proposed by Book (1990) assumed small relative deformation hence are not applicable to continuum arms that can exhibit large omni-directional deformation. Xu and Simaan (2010) utilized elliptic integrals to derive kinematics, statics, and shape restoration of continuum arms but only a single continuum section was considered. Rone and Ben-Tzvi (2014) employed Kane's method to derive dynamics for tendon-actuated multisegment (technically a single section as the segments are kinematically coupled) continuum arms. Trivedi et al. (2008a) proposed a planar model for multisection 
continuum arms and validated only for static poses. This approach has not been extended to model spatial dynamics of variable length multisection continuum arms.

Unlike their rigid counterparts, the displacement between any two points within a continuum arm is not fixed. As a result of their inherent large deformations, points along a continuum arms have varying relative position, orientation, and linear and angular velocities. To account for this continuous and deforming nature, continuum arm Equations of Motion (EoM) are derived from energy based methods such as the Lagrangian formulation.

Although there has been an impressive amount of research in prototypes, the advancements in modeling and control of variable length continuum arms, particularly dynamic modeling, lags behind. This is mainly because of the complex and highly nonlinear kinematics and dynamics associated with this class of continuum arms. Theoretical models for inextensible, unidimensional, cable like mechanisms were proposed by Mochiyama (2006) but multisection continuum arms have multiple DoF. As shown in Figures 1a and 1c, upon actuation the variable length continuum sections of the prototype arms are deformed in circular arc shapes. The kinematic model proposed by Jones and Walker (2006a) accurately calculates the arc parameters that describe this circular arc shape in joint-space variables (i.e., actuator lengths). However, due to the highly nonlinear, complex expressions and numerical instabilities, it is challenging to extend the parametric kinematics to dynamic analysis. Tatlicioglu et al. (2007a,b) proposed a dynamic model for the OctArm (Grissom et al., 2006) extensible continuum manipulator. However, these planar dynamic models did not account for the geometrical coupling between curve parameters and joint space variables (Godage et al., 2011b) and were not experimentally validated.

Godage et al. (2011a,b) proposed spatial dynamics for a single, variable length continuum section and experimentally validated for a PMA actuated continuum section in (Godage et al., 2012b). The modal $^{1}$ kinematics for variable length multisection continuum arms proposed by Godage et al. (2011c, 2015) combined the structural accuracy of curve parametric models (Jones and Walker, 2006a) and numerical efficiency and stability of modal methods (Shabana, 2005). Further, much of the work on variable multisection continuum arms so far focused on slow moving applications where static models and kinematic control were sufficient (Jones and Walker, 2006b). But increasingly continuum robots are, and surely will be, affected by dynamics for applications such as obstacle avoidance in dynamic environments, inspection operations, and object manipulation tasks which requires increased performance. Hence there is the need for scalable, numerically efficient, and structurally accurate spatial dynamic models for variable length multisection continuum arms.

Derived from modal kinematics (Godage et al., 2011c, 2015) and extending previous work on single continuum section dynamics, this paper presents a new spatial dynamic model for variable length multisection continuum arms. The EoM are derived in a recursive form and produce computationally efficient results without the numerical instabilities. The proposed method is directly derived in joint space without intermediate morphological transformations and therefore provides enhanced physical insight into the practical mechanics of continuum arms. The dynamic simulation results are then compared and validated against the PMA actuated variable length multisection continuum arm shown in Figure 1c. The proposed model can be extended to structurally similar multisection continuum arms of any variable length actuator type.

The layout of this paper is as follows. The methodology of the dynamics approach for an arbitrarily long, variable section length continuum arm is presented in Section 2 with a review of modal kinematics in the beginning. The development of an efficient, recursive computational scheme for formulating the dynamics is then developed in Section 3. Details of the prototype arm, simulation model, and adaptations are presented in Section 4. Simulation results and model validation against the prototype arm dynamics are detailed in Section 5. A discussion summarizing the previous related work highlighting the benefits of the proposed modal dynamics is given in Section 6 followed by conclusions in Section 7 .

\section{Methodology}

In this section, the development of a dynamic model for a variable length multisection continuum arm (similar to the prototype shown in Figure 1) with $N$ sections is detailed. The section-wise Lagrangian formulation presented here lays the foundation for a recursive computational scheme to compute the final EoM. Mathematical variables utilized throughout the paper are enumerated in Table 1.

\footnotetext{
${ }^{1}$ The term "modal" in (Godage et al., 2011c) depicts that the parameter variation of HTM elements are approximated by modal shape functions analogous to (Chirikjian and Burdick, 1994) and (Shabana, 2005).
} 
Table 1: Nomenclature of Mathematical Symbols

\begin{tabular}{|c|c|}
\hline Symbol & Definition \\
\hline$i$ & Continuum section index $\sharp$ \\
\hline$r_{i}, L_{i 0}$ & Radius and unactuated length of continuum section \\
\hline$l_{i j}$ & $j^{\text {th }}$ actuator length change, $j \in\{1,2,3\}$ \\
\hline$q$ & Complete joint space vector of the arm* \\
\hline $\boldsymbol{q}_{i}, \boldsymbol{q}^{i}$ & Joint space vector of, and up to $i^{t h}$ continuum section \\
\hline$\left\{O_{i}\right\},\left\{O_{i}^{\prime}\right\}$ & Base and moving coordinate frames \\
\hline$\{O\}$ & Task coordinate frame \\
\hline$\xi_{i}$ & Scalar denoting points along the of $i^{t h}$ continuum section \\
\hline $\mathbf{T}_{i}$ & $\mathrm{MHTM}^{\dagger}$ of the $i^{\text {th }}$ continuum section relative to $\left\{O_{i}\right\}$ \\
\hline $\boldsymbol{p}_{i}, \mathbf{R}_{i}$ & Position vector and rotation matrix of $\mathbf{T}_{i}$ \\
\hline $\begin{array}{l}\mathbf{T}_{i}^{J} \\
\mathbf{T}^{i}\end{array}$ & Constant $\mathrm{HTM}^{\ddagger}$ to account for position/orientation offsets at $i^{\text {th }}$ section joint \\
\hline$\Psi_{i}, \Theta_{i}$ & \\
\hline$\xi, \xi^{i}$ & Vector of scalar coefficients of the entire arm, and up to $i^{\text {th }}$ section. \\
\hline$m_{i}$ & Mass of the continuum section \\
\hline$\rho_{i}$ & Linear mass density \\
\hline $\mathcal{K}, \mathcal{K}_{i}$ & Total and $i^{t h}$ section kinetic energy \\
\hline $\mathcal{P}, \mathcal{P}_{i}$ & Total and $i^{\text {th }}$ section potential energy \\
\hline $\mathbf{H}_{i}^{v}, \mathbf{H}_{i}^{\omega}$ & Linear and angular Hessians relative to $\left\{O_{i}^{\prime}\right\}$ at $\xi_{i}$. \\
\hline $\mathcal{M}_{i}^{v}, \mathcal{M}_{i}^{\omega}$ & Linear and angular inertia matrices of a slice of continuum section at $\xi_{i}$ \\
\hline $\mathbf{M}, \mathbf{C}$ & Complete generalized inertia and Coriolis/Centrifugal force matrices of entire arm \\
\hline $\mathbf{M}_{i}^{v}, \mathbf{M}_{i}^{\omega}$ & Generalized linear and angular inertia matrices \\
\hline $\mathbf{C}_{i}^{v}, \mathbf{C}_{i}^{\omega^{2}}$ & Linear and angular Coriolis/Centrifugal force matrices \\
\hline$G, G_{i}$ & Complete and $i^{t h}$ section conservative force vectors \\
\hline $\mathbf{D}, \mathbf{D}_{i}$ & Complete and $i^{t h}$ section damping coefficient matrices. \\
\hline$v_{i}, \omega_{i}$ & Linear and angular velocities relative to $\left\{O_{i}^{\prime}\right\}$ at $\xi_{i}$ \\
\hline $\mathbf{J}_{i}^{v}, \mathbf{J}_{i}^{\omega}$ & Linear and angular Jacobians at $\xi_{i}$ relative to $\left\{O_{i}^{\prime}\right\}$ \\
\hline $\mathbf{K}_{i}^{e}$ & Elastic stiffness coefficient matrix \\
\hline$\tau_{e}$ & Complete input force vector in the joint space \\
\hline $\mathbf{I}_{3}$ & Rank 3 identity matrix \\
\hline
\end{tabular}

$\sharp$ Subscript $i$ denotes the $i^{\text {th }}$ continuum section parameters

$*$ Vectors are denoted by boldface lowercase italics (i.e., $\boldsymbol{q}_{j}$ ) and elements are denoted by regular lowercase italics (i.e., $q_{j}$ ).

$\ddagger$ Homogeneous transformation matrix (HTM). Matrices are denoted by boldface uppercase letters (i.e., M)

$\dagger$ Modal homogeneous transformation matrix (MHTM). 


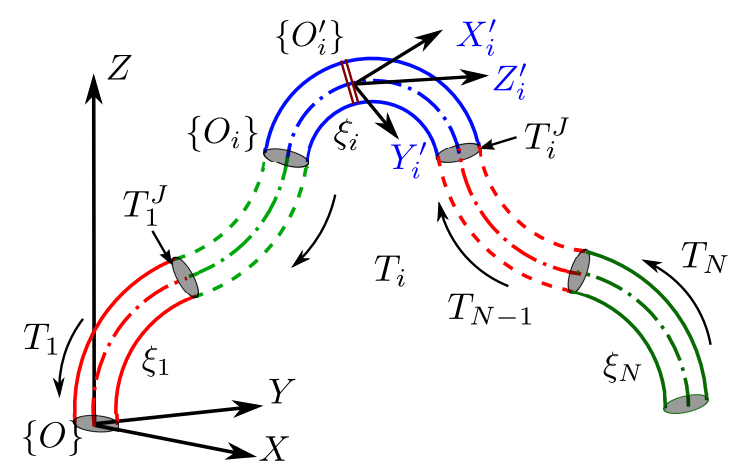

Figure 2: Schematic of a multisection continuum arm with arbitrary $N$ continuum sections. The base section (indexed 1) coincides with $\{O\}$. The base coordinate frame, $\left\{O_{i}\right\}$, and moving coordinate frame at $\xi_{i}$ of the $i^{\text {th }}$ section, $\left\{O_{i}^{\prime}\right\}$, are also shown.

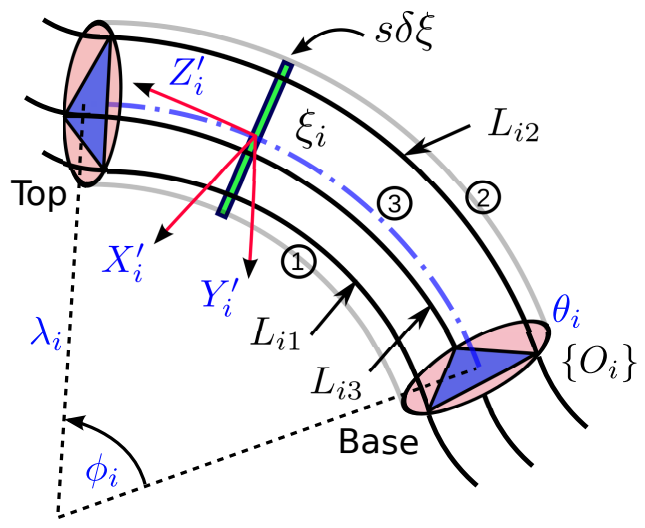

(a)

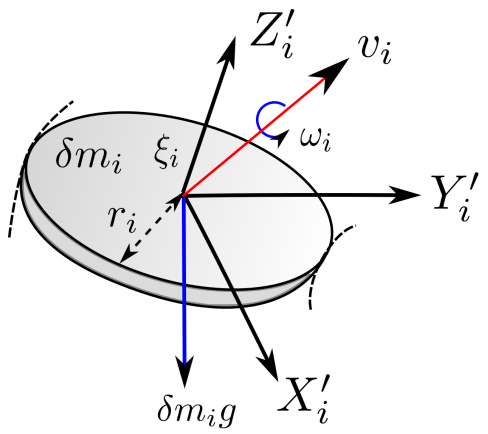

(b)

Figure 3: (a) Schematical illustration of the infinitesimally thin slice at $\xi_{i}$ on any $i^{\text {th }}$ continuum section along with curve parameters $\left\{\lambda_{i}, \phi_{i}, \theta_{i}\right\}$ (listed in Appendix A.1), actuator variables, $\left\{O_{i}\right\}$, and $\left\{O_{i}^{\prime}\right\}$, (b) Velocities and forces acting on the thin slice with respect to $\left\{O_{i}^{\prime}\right\}$.

\subsection{Variable Length Multisection Continuum Arm System Model}

Figure 2 shows the schematic for a variable length, $N$ section, continuum arm. Without losing generality, the widely used and optimal (for spatial operation), three-actuator configuration is considered per continuum section similar to the prototypes shown in Figure 1. One notable difference between this class of continuum arms and the more traditional tendon actuated continuum arms is that the actuator lengths of continuum sections are kinematically independent. In tendon actuated arms, the tendons run along the length of the arm and therefore actuation of preceding sections (assuming no slacking of tendons) affect the tendon lengths of subsequent sections.

Consider the schematic of any $i^{\text {th }}$ continuum section of the arm as shown in Figure $3 \mathrm{a}$. It consists of three mechanically identical variable length actuators with $L_{i 0} \in \mathbb{R}$ and $l_{i j}(t) \in \mathbb{R}$, where $j \in\{1,2,3\}$ and $t$ is the time. Hence the length of an actuator at any time is $L_{i j}=L_{i 0}+l_{i j}(t)$. In this way, both extensions, i.e., $l_{i j} \in \mathbb{R}_{0}^{+}$and contractions, i.e., $l_{i j} \in \mathbb{R}_{0}^{-}$can be described (Walker et al., 2006). The actuators are fixed $\frac{2 \pi}{3}$ rads apart and are mechanically constrained to actuate at a distance $r_{i}$, parallel to the neutral axis. The $l_{11}$ actuator is aligned with the $X$ axis of $\{O\}$. Similarly, $l_{i 1}$ actuator is aligned with the $X_{i}$ axis of $\left\{O_{i}\right\}$. Note that $L_{i 0}>0$ and $r_{i}>0$ are known constant design parameters of the continuum sections.

\subsection{Review of Modal Kinematics and Dynamics}

In dynamics derived in this paper is based on the modal kinematics proposed by Godage et al. (2011c, 2015). It provides an intuitive, stable, efficient, structurally accurate, and scalable kinematics for variable length continuum arms. Further, the kinematics are computed directly in the joint-space without 
intermediary transformations thus avoiding nonlinear morphological mappings. As the individual continuum sections are kinematically independent, the modal homogeneous transformation matrix (MHTM) of $\left\{O_{i}^{\prime}\right\}$ at $\xi_{i}$ of any $i^{t h}$ continuum section, $\mathbf{T}_{i} \in S E(3)$, with respect to $\left\{O_{i}\right\}$ is given by

$$
\mathbf{T}_{i}\left(\xi_{i}, \boldsymbol{q}_{i}\right)=\left[\begin{array}{cc}
\mathbf{R}_{i}\left(\xi_{i}, \boldsymbol{q}_{i}\right) & \boldsymbol{p}_{i}\left(\xi_{i}, \boldsymbol{q}_{i}\right) \\
\mathbf{0} & 1
\end{array}\right]
$$

where $\boldsymbol{q}_{i}=\left[l_{i 1}, l_{i 2}, l_{i 3}\right]^{T} \in \mathbb{R}^{3}, \xi_{i} \in[0,1]$ is a scalar coefficient that defines the points along the length of the neutral axis $\left(\xi_{i}=0\right.$ is the base and $\xi_{i}=1$ is the tip), $\mathbf{R}_{i} \in S O(3)$, and $\boldsymbol{p}_{i} \in \mathbb{R}^{3}$. For clarity, the complete curve parametric HTM, from which the MHTM is derived, is included in Appendix A.1. The complete MHTM is listed in Appendix A.2.

Employing basic coordinate transformations, $\mathbf{T}^{i} \in S E(3)$ relative to $\{O\}$ is calculated as

$$
\begin{aligned}
\mathbf{T}^{i}\left(\boldsymbol{\xi}^{i}, \boldsymbol{q}^{i}\right) & =\prod_{k=1}^{i}\left\{\mathbf{T}_{k}\left(\boldsymbol{q}_{k}, \xi_{k}\right) \mathbf{T}_{k}^{J}\right\} \\
& =\left[\begin{array}{cc}
\Theta_{i}\left(\boldsymbol{\xi}^{i}, \boldsymbol{q}^{i}\right) & \Psi_{i}\left(\boldsymbol{\xi}^{i}, \boldsymbol{q}^{i}\right) \\
\mathbf{0} & 1
\end{array}\right]
\end{aligned}
$$

where $\Theta_{i} \in S O(3), \Psi_{i} \in \mathbb{R}^{3}, \boldsymbol{q}^{i}=\left[\boldsymbol{q}_{1}, \boldsymbol{q}_{2}, \cdots, \boldsymbol{q}_{i}\right] \in \mathbb{R}^{3 i}$ and $\boldsymbol{\xi}^{i}=\left[\xi_{1}, \xi_{2}, \cdots, \xi_{i}\right] \in \mathbb{R}^{i}$ and $\xi_{k}=1, \forall k<i$ (i.e., the preceding sections) and $\xi_{k}=0, \forall k>i$ (i.e., the successive sections).

Assuming continuum sections are made up of infinitesimally thin slices, Godage et al. $(2011 \mathrm{a}, \mathrm{b})$ proposed an integral Lagrangian formulation based modal dynamics for single section variable length continuum arms. Therein, first the kinetic energy (KE) and potential energy (PE) are derived for a thin slice and then the total energies are found by integrating along the length of the arm. The Lagrangian is then utilized to derive the EoM of the continuum section.

\subsection{Equations of Motion for Variable Length Multisection Continuum Arms}

Based on the modal kinematics (Godage et al., 2011c, 2015), the integral Lagrangian approach used on single continuum sections (Godage et al., 2011a) is extended herein for formulating the dynamics of multisection continuum arms. However, the complex kinematics and integral nature of the procedure prevent direct extension of the integral derivation proposed in (Godage et al., 2011a) to multisection continuum arms. Therefore we propose a section-wise formulation technique. In developing this model, for any $i^{\text {th }}$ continuum section it is assumed that;

AS1 Continuum sections always maintain a circular arc shape

This implies that continuum sections are torsionally stiff and not subjected to "large" external forces.

AS2 Continuum sections have no supporting backbones

The variable length actuators provide the only structural support.

AS3 Continuum sections have symmetric cross sections about the neutral axis of the arm As a result, the center of mass is located at the center of the cross section.

AS4 Continuum sections are kinematically independent

The actuation of one continuum section does not affect any other sections.

AS5 The actuators are only subject to length change and therefore maintaining $r_{i}$ and $\frac{2 \pi}{3}$ clearance This assumption further ensures the assumption AS1.

AS6 Continuum sections have constant $m_{i}$ and variable but uniform linear density $\left(\rho_{i}\right)$ The effect of air pressure changes is neglected. The length variation during operation cause $\rho_{i}$ to vary as $m_{i}$ is constant.

The validity of these assumptions for the prototype continuum arm (shown in Figure 1c) is discussed in Section 4.2. For ease of presentation, the particular mathematical operators used in the paper are enumerated in Table 2. 


\begin{tabular}{|c|c|}
\hline Operator & Definition \\
\hline$\nabla_{q}(\quad)$ & Partial derivative with respect to vector $\boldsymbol{q}$ \\
\hline & Partial derivative with respect to variable $q_{x} \in \boldsymbol{q}$ where $q_{x} \in \mathbb{R}$ \\
\hline & $x^{t h}$ column vector of the enclosed matrix \\
\hline & $x^{t h}$ element of the enclosed vector \\
\hline & $(x, y)^{t h}$ element of the enclosed matrix \\
\hline( & $(x, y)^{t h}$ vector of the enclosed Hessian of a vector \\
\hline( & Form the velocity vector from skew symmetric angular velocity matrix* \\
\hline 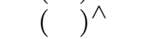 & Form skew symmetric angular velocity matrix from angular velocity vector* \\
\hline & Integration from 0 to 1 with respect to $\xi_{i}$ \\
\hline & Replace the last column of the matrix with zeros \\
\hline $\operatorname{Diag}()$ & Form a diagonal matrix from the enclosed vector as the principal diagonal \\
\hline $\mathbb{T}_{2}()$ & Sum first two elements of the principal diagonal of $3 \times 3$ matrix \\
\hline $\mathbb{V}(\quad)$ & Column-wise vectorization operation on a matrix \\
\hline
\end{tabular}

* Definitions are given in Appendix B.

If the total KE and PE of the entire arm shown in Figure 2 is known, then the complete Lagrangian is given by $\mathcal{L}=\mathcal{K}-\mathcal{P}$. Without considering the dissipative terms, the ideal generalized EoM are derived from the Lagrangian as $\frac{d}{d t} \nabla_{\dot{\boldsymbol{q}}}^{T} \mathcal{L}-\nabla_{\boldsymbol{q}}^{T} \mathcal{L}$ and in classical compact matrix form presented as

$$
\mathbf{M} \ddot{\boldsymbol{q}}+\mathbf{C} \dot{\boldsymbol{q}}+\boldsymbol{G}=\tau_{e}
$$

where $\boldsymbol{\tau}_{e} \in \mathbb{R}^{3 N}$. Physically, $\boldsymbol{\tau}_{e}$ is the tensile or compressive forces generated by the variable length actuators.

Terms of the EoM given by Equation (3) contain the cumulative contributions from each continuum section of the arm. Therefore, the respective terms of Equation (3) are decomposed herein to sectionwise dynamic contributions to obtain a general representation that can be incorporated into a recursive computation scheme. The following theorems are used to integrate section-wise component contributions into the final EoM.

Theorem 1. Consider a general (rigid or continuum) $N$-link serial arm with an actuated joint space vector $\boldsymbol{q} \in \mathbb{R}^{u}$. Then the complete generalized inertia matrix, $\mathbf{M}=\left(\sum_{i=1}^{N} \mathbf{M}_{i}\right) \in \mathbb{R}^{u \times u}$ where $\mathbf{M}_{i} \in$ $\mathbb{R}^{u \times u}$ are individual generalized inertial matrices corresponding to any $i^{\text {th }}$ link.

Proof. Let $\boldsymbol{q}_{i}$ and $\boldsymbol{q}=\left[\boldsymbol{q}_{1}, \boldsymbol{q}_{2}, \cdots, \boldsymbol{q}_{N}\right] \in \mathbb{R}^{u}$ be the $i^{\text {th }}$ link and complete joint space vectors respectively. The spatial location of any $i^{t h}$ link is a function of preceding links and $\boldsymbol{q}^{i}=\left[\boldsymbol{q}_{1}, \boldsymbol{q}_{2}, \cdots, \boldsymbol{q}_{i}\right] \in \mathbb{R}^{w}(w \leq u)$ denotes the joint space vector up to the $i^{t h}$ link. The $\mathbf{M}$ can be related to the total KE of the arm, $\mathcal{K} \in \mathbb{R}$, as

$$
\mathcal{K}(\boldsymbol{q}, \dot{\boldsymbol{q}})=\frac{1}{2} \dot{\boldsymbol{q}}^{T} \mathbf{M} \dot{\boldsymbol{q}}
$$

Similarly, the $\mathbf{M}_{i}$ and $\mathcal{K}_{i}$ are related as

$$
\mathcal{K}_{i}\left(\boldsymbol{q}^{i}, \dot{\boldsymbol{q}}^{i}\right)=\frac{1}{2} \dot{\boldsymbol{q}}^{T} \mathbf{M}_{i} \dot{\boldsymbol{q}}
$$

Note that, $\left[\mathbf{M}_{i}\right]_{j k}=0 \forall\{j, k\}>w$ because $\boldsymbol{q}^{i} \mapsto \mathcal{K}_{i}\left(\boldsymbol{q}^{i}\right)$ and kinematic independence of continuum sections (assumption AS4 in Section 2.3).

The $\mathcal{K}$ is the sum of KE's of all the arm links and can be written as

$$
\mathcal{K}=\sum_{i=1}^{N} \mathcal{K}_{i}
$$

Substituting $\mathcal{K}$ from Equation (4) and $\mathcal{K}_{i}$ from Equation (5) into Equation (6) yields 


$$
\frac{1}{2} \dot{\boldsymbol{q}}^{T} \mathbf{M} \dot{\boldsymbol{q}}=\sum_{i=1}^{N}\left(\frac{1}{2} \dot{\boldsymbol{q}}^{T} \mathbf{M}_{i} \dot{\boldsymbol{q}}\right)=\frac{1}{2} \dot{\boldsymbol{q}}^{T}\left(\sum_{i=1}^{N} \mathbf{M}_{i}\right) \dot{\boldsymbol{q}}
$$

From the Equation (7) it follows that

$$
\mathbf{M}=\sum_{i=1}^{N} \mathbf{M}_{i}
$$

Proof completed.

Proposition 2. From Theorem 1, the complete centrifugal/Coriolis force matrix, $\mathbf{C}=\left(\sum_{i=1}^{N} \mathbf{C}_{i}\right) \in$ $\mathbb{R}^{u \times u}$ where $\mathbf{C}_{i} \in \mathbb{R}^{u \times u}$ are individual centrifugal/Coriolis force matrices corresponding to any $i^{\text {th }}$ link. Proof. Define $[\mathbf{C}]_{j k} \in \mathbb{R}$ as

$$
[\mathbf{C}]_{k j}=\sum_{h=1}^{u} \Gamma_{k j h}(\mathbf{M}) \dot{q}_{h}
$$

where $\mathbf{M} \in \mathbb{R}^{u \times u}, \Gamma_{k j h}(\mathbf{M})=\frac{1}{2}\left([\mathbf{M}]_{k j, h}+[\mathbf{M}]_{k h, j}-[\mathbf{M}]_{h j, k}\right) \in \mathbb{R}$ is the respective Christoffel symbol, $q_{h} \in \boldsymbol{q}$, and $q_{h} \in \mathbb{R}$.

Substitute $\mathbf{M}$ from Equation (8) into Equation (9) to obtain

$$
[\mathbf{C}]_{k j}=\sum_{h=1}^{u} \Gamma_{k j h}\left(\sum_{i=1}^{N} \mathbf{M}_{i}\right) \dot{q}_{h}=\sum_{i=1}^{N}\left\{\sum_{h=1}^{u} \Gamma_{k j h}\left(\mathbf{M}_{i}\right) \dot{q}_{h}\right\}
$$

From the definition of $[\mathbf{C}]_{k j}$ given in Equation (9) it follows that the Coriolis/centrifugal force matrix elements of the $i^{t h} \operatorname{link}\left[\mathbf{C}_{i}\right]_{k j}=\sum_{h=1}^{u} \Gamma_{k j h}\left(\mathbf{M}_{i}\right) \dot{q}_{h}$ and $\Gamma_{k j h}\left(\mathbf{M}_{i}\right)=0 \forall\{k, j, h\}>w$ because $\boldsymbol{q}^{i} \mapsto \mathbf{M}_{i}\left(\boldsymbol{q}^{i}\right)$ and kinematic independence of continuum sections (assumption AS4 in Section 2.3). Consequently $\left[\mathbf{C}_{i}\right]_{k j}=\sum_{h=1}^{w} \Gamma_{k j h}\left(\mathbf{M}_{i}\right) \dot{q}_{h}$ and Equation (10) can be rewritten as

$$
[\mathbf{C}]_{k j}=\sum_{i=1}^{N}\left\{\sum_{h=1}^{w} \Gamma_{k j h}\left(\mathbf{M}_{i}\right) \dot{q}_{h}\right\}=\sum_{i=1}^{N}\left[\mathbf{C}_{i}\right]_{k j}
$$

By element mapping of Equation (11) therefore

$$
\mathbf{C}=\sum_{i=1}^{N} \mathbf{C}_{i}
$$

Proof completed.

Theorem 3. Consider a serially-linked arm having $N$ links (rigid or continuum) with an actuated joint space vector $\boldsymbol{q} \in \mathbb{R}^{u}$. Then the complete conservative force vector, $\boldsymbol{G}=\left(\sum_{i=1}^{N} \boldsymbol{G}_{i}\right) \in \mathbb{R}^{u}$ where $\boldsymbol{G}_{i} \in \mathbb{R}^{u}$ are individual conservative force vector vectors corresponding to any $i^{\text {th }}$ link.

Proof. The total arm PE, $\mathcal{P} \in \mathbb{R}$, and $\boldsymbol{G}$ are related as

$$
\nabla_{\boldsymbol{q}}^{T} \mathcal{P}(\boldsymbol{q})=\boldsymbol{G}
$$

Similarly, $i^{\text {th }}$ link PE, $\mathcal{P}_{i} \in \mathbb{R}$, and $\boldsymbol{G}_{i}$ are related as

$$
\nabla_{\boldsymbol{q}}^{T} \mathcal{P}_{i}\left(\boldsymbol{q}^{i}\right)=\boldsymbol{G}_{i}
$$


where $\left[\boldsymbol{G}_{i}\right]_{k}=0 \forall k>w$ because $\boldsymbol{q}^{i} \mapsto \boldsymbol{G}_{i}\left(\boldsymbol{q}^{i}\right)$ and kinematic independence of continuum sections (assumption AS4 in Section 2.3).

The total PE is cumulative of PE's of every arm link and therefore

$$
\mathcal{P}=\sum_{i=1}^{N} \mathcal{P}_{i}
$$

Apply $\nabla_{\boldsymbol{q}}^{T}$ to both sides of Equation (15) and substitute Equations (13) and (14) to obtain

$$
\boldsymbol{G}=\sum_{i=1}^{N}\left(\nabla_{\boldsymbol{q}}^{T} \mathcal{P}_{i}\right)=\sum_{i=1}^{N} \boldsymbol{G}_{i}
$$

Proof completed.

Now that the relation of section-wise components to the final EoM given by Equation (3) is established. Next, the section-wise Lagrangian formulation to derive these contributions is presented.

\subsection{The Lagrangian Formulation of Any $i^{\text {th }}$ Continuum Section}

This section, without losing generality, presents the method to compute $\mathbf{M}_{i} \in \mathbb{R}^{3 N \times 3 N}, \mathbf{C}_{i} \in \mathbb{R}^{3 N \times 3 N}$, and $\boldsymbol{G}_{i} \in \mathbb{R}^{3 N}$ from the $\mathrm{KE}$ and $\mathrm{PE}$ of any $i^{\text {th }}$ continuum section. For ease of presentation, applying Theorem 1 to linear and angular KE's of the continuum section separately, $\mathbf{M}_{i}$ is further expanded as

$$
\mathbf{M}_{i}=\mathbf{M}_{i}^{v}+\mathbf{M}_{i}^{\omega}
$$

where $\mathbf{M}_{i}^{v} \in \mathbb{R}^{3 N \times 3 N}$ and $\mathbf{M}_{i}^{\omega} \in \mathbb{R}^{3 N \times 3 N}$. The partial derivatives of $\mathbf{M}_{i}$ are used to compute $\mathbf{C}_{i}$ as shown in Proposition 2.

\subsubsection{Position, Orientation and Velocity of a Thin Slice}

Consider an infinitesimally thin slice of any $i^{\text {th }}$ continuum section at $\xi_{i}$ as shown in Figure 3a. As with any serially connected structure, the position, orientation, and velocity of the $i^{t h}$ continuum section only depends on all the preceding continuum sections hence $\xi_{k}=1 \forall k<i$ and $\xi_{k}=0 \forall k>i$ (see Figure 2)

(Godage et al., 2011c). The $\omega_{i} \in \mathbb{R}^{3}$ and $v_{i} \in \mathbb{R}^{3}$ at any point $\xi_{i}$ along the neutral axis relative to $\left\{O_{i}^{\prime}\right\}$ is given by

$$
\begin{aligned}
\boldsymbol{\omega}_{i}\left(\xi_{i}, \boldsymbol{q}^{i}\right) & =\left(\Theta^{T} \dot{\Theta}_{i}\right)^{\vee}=\mathbf{J}_{i}^{\omega}\left(\xi_{i}, \boldsymbol{q}^{i}\right) \dot{\boldsymbol{q}} \\
v_{i}\left(\xi_{i}, \boldsymbol{q}^{i}\right) & =\Theta_{i}^{T} \dot{\Psi}_{i}=\mathbf{J}_{i}^{v}\left(\xi_{i}, \boldsymbol{q}^{i}\right) \dot{\boldsymbol{q}}
\end{aligned}
$$

where $\mathbf{J}_{i}^{\omega}=\left(\Theta_{i}^{T} \nabla_{\boldsymbol{q}} \Theta_{i}\right)^{\vee} \in \mathbb{R}^{3 \times 3 N}$ and $\mathbf{J}_{i}^{v}=\left(\Theta_{i}^{T} \nabla_{\boldsymbol{q}} \Psi_{i}\right) \in \mathbb{R}^{3 \times 3 N}$.

\subsubsection{Kinetic Energy, $\mathrm{M}_{i}$ and $\mathrm{C}_{i}$ Matrices}

Following the approach reported by Godage et al. (2011b), the mass of an infinitesimally thin slice (see Figure $3 \mathrm{~b}$ ) is derived as $\delta m_{i}=m_{i} \delta \xi_{i}$ due to the constant mass and uniform linear density (assumption AS6 in Section 2.3). The center of mass of the slice lies at its geometric center and $I_{x x}=I_{y y}$ because of the symmetric cross section (assumption AS3 in Section 2.3) where $I_{x x}$ and $I_{y y}$ are the moments of inertia about the $X_{i}^{\prime}$ and $Y_{i}^{\prime}$ axes respectively. Hence $\mathcal{M}_{i}^{v}=m_{i} \delta \xi_{i} \mathbf{I}_{3}$ and $\mathcal{M}_{i}^{\omega}=I_{x x} \delta \xi_{i} \operatorname{Diag}(1,1,2)$ derived from $I_{z z}=I_{x x}+I_{y y}=2 I_{x x}$ (perpendicular axis theorem) where $I_{z z}$ is the moments of inertia about the $Z_{i}^{\prime}$ axis.

The angular $\left(\delta \mathcal{K}_{i}^{\omega} \in \mathbb{R}\right)$ and linear $\left(\delta \mathcal{K}_{i}^{v} \in \mathbb{R}\right)$ KE's for the thin slice can be written utilizing $\mathbf{J}_{i}^{\omega}$ from Equation (18), $\mathbf{J}_{i}^{v}$ from Equation (19), $\mathcal{M}_{i}^{v}$, and $\mathcal{M}_{i}^{\omega}$ as

$$
\begin{aligned}
\delta \mathcal{K}_{i}^{\omega} & =\frac{1}{2} \omega_{i}^{T} \mathcal{M}_{i}^{\omega} \omega_{i}=\frac{1}{2} \dot{\boldsymbol{q}}^{T} \delta \mathbf{M}_{i}^{\omega}\left(\xi_{i}, \boldsymbol{q}^{i}\right) \dot{\boldsymbol{q}} \\
\delta \mathcal{K}_{i}^{v} & =\frac{1}{2} v_{i}^{T} \mathcal{M}_{i}^{v} v_{i}=\frac{1}{2} \dot{\boldsymbol{q}}^{T} \delta \mathbf{M}_{i}^{v}\left(\xi_{i}, \boldsymbol{q}^{i}\right) \dot{\boldsymbol{q}}
\end{aligned}
$$


where $\delta \mathbf{M}_{i}^{\omega}=\mathbf{J}_{i}^{\omega T} \mathcal{M}_{i}^{\omega} \mathbf{J}_{i}^{\omega}$ and $\delta \mathbf{M}_{i}^{v}=\mathbf{J}_{i}^{v T} \mathcal{M}_{i}^{v} \mathbf{J}_{i}^{v}$ are the angular and linear generalized inertia matrices of the thin slice.

By integrating with respect to $\xi_{i}, \mathbf{M}_{i}^{v}$ and $\mathbf{M}_{i}^{\omega}$ can now be computed as

$$
\begin{aligned}
& \mathbf{M}_{i}^{\omega}\left(\boldsymbol{q}^{i}\right)=\int_{\xi_{i}}\left(\mathbf{J}_{i}^{\omega T} \mathcal{M}_{i}^{\omega} \mathbf{J}_{i}^{\omega}\right) \\
& \mathbf{M}_{i}^{v}\left(\boldsymbol{q}^{i}\right)=\int_{\xi_{i}}\left(\mathbf{J}_{i}^{v T} \mathcal{M}_{i}^{v} \mathbf{J}_{i}^{v}\right)
\end{aligned}
$$

Partial derivatives of $\mathbf{M}_{i}$ required to derive the $\mathbf{C}_{i}$ can now be computed from Equations (22) and (23).

\subsubsection{Potential Energy and $G_{i}$ Vector}

Any $i^{\text {th }}$ continuum section is subjected to gravitational $\mathrm{PE}, \mathcal{P}_{i}^{g} \in \mathbb{R}$, and elastic $\mathrm{PE}, \mathcal{P}_{i}^{e} \in \mathbb{R}$. Assuming the continuum arm is made up of infinitesimally thin slices, the gravitational $\mathrm{PE}\left(\delta \mathcal{P}_{i}^{g} \in \mathbb{R}\right)$ of a slice at $\xi_{i}$ is

$$
\delta \mathcal{P}_{i}^{g}\left(\xi_{i}, \boldsymbol{q}^{i}\right)=\left(m_{i} \delta \xi\right) \Psi_{i}^{T} \boldsymbol{g}
$$

where $\boldsymbol{g}=[0,0, g]^{T} \in \mathbb{R}^{3}$ is the gravity vector in $\{O\}$.

The total gravitational PE of the continuum section is then determined as

$$
\mathcal{P}_{i}^{g}\left(\boldsymbol{q}^{i}\right)=\int_{\xi_{i}} \delta \mathcal{P}_{g}=m_{i}\left(\int_{\xi_{i}} \Psi_{i}^{T}\right) \boldsymbol{g}
$$

The elastic PE of any continuum section is due to the axial elastic deformation of variable length actuators (e.g. PMA's) during operation. The elastic PE of the $i^{t h}$ continuum section is given by

$$
\mathcal{P}_{i}^{e}\left(\boldsymbol{q}_{i}\right)=\frac{1}{2} \boldsymbol{q}_{i}^{T} \mathbf{K}_{i}^{e} \boldsymbol{q}_{i}
$$

where $\mathbf{K}_{i}^{e}=\operatorname{Diag}\left\{K_{i 1}, K_{i 2}, K_{i 3}\right\}$ and $K_{i j} \forall j \in\{1,2,3\}$ are the elastic stiffness coefficients of the three variable length actuators.

The $\boldsymbol{G}_{i}$ is then calculated utilizing Equations (25) and (26) as

$$
\boldsymbol{G}_{i}\left(\boldsymbol{q}^{i}\right)=\nabla_{\boldsymbol{q}}^{T}\left(\mathcal{P}_{i}^{g}+\mathcal{P}_{i}^{e}\right)
$$

This completes the theoretical deriving of the contributions from any $i^{\text {th }}$ continuum section to the final EoM.

\section{Development of an Efficient Computational Scheme}

This section proposes an efficient recursive implementation for computing the section-wise contributions developed in Section 2.4 toward the complete EoM given by Equation (3). The lack of accurate spatial dynamic models for variable length multisection continuum arms is because of many resulting integral expressions containing complex laborious terms, i.e., consider Equations (22) and (23). The complexity and size of these integrands substantially increase as the number of continuum sections, $N$, grows. Bertails (2009) proposed efficient dynamics for constant length helical shapes for computer graphics. However the approach in the paper was developed for simulating passive flexible objects and does not account for actuators used in robots. Also, Hollerbach (1980) and Featherstone (2008) among others proposed recursive computational schemes that are limited to rigid arms.

Similar to the procedure for the single continuum arm case reported in (Godage et al., 2011a), the integrals can be precalculated symbolically. However, unlike the single section continuum arms, integrable components of expressions are separated by complex non-integrable (terms independent of the variable of integration) components. Hence in the multisection arm case, additional algebraic relationships are used to simplify and bring integrable terms together so those can be precomputed to increase the computational efficiency. In order to accomplish this, first the spatial displacement, corresponding velocity Jacobians, and Hessians are recursively implemented. Utilizing these, recursive relationships are then derived for EoM contributions, $\mathbf{M}_{i}, \mathbf{C}_{i}$, and $\boldsymbol{G}_{i}$ from any $i^{t h}$ section. Finally, the results from each continuum section are iteratively summed up through Theorems 1, 2 and 3 to obtain the final EoM. 


\subsection{Recursive Implementation of Body Velocities, Jacobians, and Hessians}

In the Lagrangian formulation, the Jacobians play a significant role to derive $\mathbf{M}_{i}$ and $\mathbf{C}_{i}$ as shown by Equations (22) and (23). In this section, recursive representations of displacements, Jacobians, and Hessians are developed.

Consider the infinitesimally thin slice at $\xi_{i}$ of the $i^{\text {th }}$ section (see Figure $3 \mathrm{a}$ ) where the spatial location is given by $\left\{O_{i}^{\prime}\right\}$. The recursive rotational matrix and position vector are given by

$$
\begin{aligned}
& \Theta_{i}\left(\xi_{i}, \boldsymbol{q}^{i}\right)=\Theta_{i-1}\left(\boldsymbol{q}^{i-1}\right) \mathbf{R}_{i}\left(\xi_{i}, \boldsymbol{q}_{i}\right) \\
& \Psi_{i}\left(\xi_{i}, \boldsymbol{q}^{i}\right)=\Psi_{i-1}\left(\boldsymbol{q}^{i-1}\right)+\Theta_{i-1} \boldsymbol{p}_{i}\left(\xi_{i}, \boldsymbol{q}_{i}\right)
\end{aligned}
$$

where the derivation is given in Appendix C.1. Note that, for ease of notation, the dependency variables are omitted from here onward.

From the definition of $\mathbf{J}_{i}^{\omega}$ given in Equation (18), relationship $\left(\mathbf{J}_{i}^{\Omega}\right)_{j}=\left(\mathbf{J}_{i}^{\omega}\right)_{j}^{\wedge}$, and noting $\boldsymbol{q}^{i}=$ $\left[\boldsymbol{q}^{i-1}, \boldsymbol{q}_{i}\right],\left(\mathbf{J}_{i}^{\Omega}\right)_{j}$ in recursive form is given by

$$
\left(\mathbf{J}_{i}^{\Omega}\right)_{j}= \begin{cases}\mathbf{R}_{i}^{T}\left(\mathbf{J}_{i-1}^{\Omega}\right)_{j} \mathbf{R}_{i} & ; q_{j} \in \boldsymbol{q}^{i-1} \\ \mathbf{R}_{i}^{T} \mathbf{R}_{i, j} & ; q_{j} \in \boldsymbol{q}_{i}\end{cases}
$$

The derivation is given in Appendix C.2.

Similarly, from the definition of $\mathbf{J}_{i}^{v}$ in Equation $(19),\left(\mathbf{J}_{i}^{v}\right)_{j}$ in recursive format is given by

$$
\left(\mathbf{J}_{i}^{v}\right)_{j}= \begin{cases}\mathbf{R}_{i}^{T}\left\{\left(\mathbf{J}_{i-1}^{v}\right)_{j}+\left(\mathbf{J}_{i}^{\Omega}\right)_{j} \boldsymbol{p}_{i}\right\} & ; q_{j} \in \boldsymbol{q}^{i-1} \\ \mathbf{R}_{i}^{T} \boldsymbol{p}_{i, j} & ; q_{j} \in \boldsymbol{q}_{i}\end{cases}
$$

The derivation is given in Appendix C.3.

Hessian matrices of the velocity relationships are used to compute $\mathbf{C}_{i}$. Taking the partial derivatives of $\left(\mathbf{J}_{i}^{\Omega}\right)_{j}$ given by Equation (30), with respect to cases $q_{k} \in \boldsymbol{q}^{i-1}$ and $q_{k} \in \boldsymbol{q}_{i}$, the recursive relationship of $\left(\mathbf{H}_{i}^{\Omega}\right)_{j k}$ is given by

$$
\left(\mathbf{H}_{i}^{\Omega}\right)_{j k}= \begin{cases}\mathbf{R}_{i}^{T}\left(\mathbf{H}_{i-1}^{\Omega}\right)_{j k} \mathbf{R}_{i} & ;\left\{q_{j}, q_{k}\right\} \in \boldsymbol{q}^{i-1} \\ \mathbf{R}_{i, k}^{T}\left(\mathbf{J}_{i-1}^{\Omega}\right)_{j} \mathbf{R}_{i}+\mathbf{R}_{i}^{T}\left(\mathbf{J}_{i-1}^{\Omega}\right)_{j} \mathbf{R}_{i, k} & ; q_{j} \in \boldsymbol{q}^{i-1}, q_{k} \in \boldsymbol{q}_{i} \\ 0 & ; q_{j} \in \boldsymbol{q}_{i}, q_{k} \in \boldsymbol{q}^{i-1} \\ \mathbf{R}_{i, k}^{T} \mathbf{R}_{i, j}+\mathbf{R}_{i}^{T} \mathbf{R}_{i, j, k} & ;\left\{q_{j}, q_{k}\right\} \in \boldsymbol{q}_{i}\end{cases}
$$

The derivation is given in Appendix C.4.

Similarly the recursive relationship of $\left(\mathbf{H}_{i}^{v}\right)_{j k}$ is derived from Equation (31) as

$$
\left(\mathbf{H}_{i}^{v}\right)_{j k}= \begin{cases}\mathbf{R}_{i}^{T}\left\{\left(\mathbf{H}_{i-1}^{v}\right)_{j}+\left(\mathbf{H}_{i-1}^{\Omega}\right)_{j} \boldsymbol{p}_{i}\right\} & ;\left\{q_{j}, q_{k}\right\} \in \boldsymbol{q}^{i-1} \\ \mathbf{R}_{i, k}^{T}\left\{\left(\mathbf{J}_{i-1}^{v}\right)_{j}+\left(\mathbf{J}_{i-1}^{\Omega}\right)_{j} \boldsymbol{p}_{i}\right\}+\mathbf{R}_{i}^{T}\left(\mathbf{J}_{i}^{\Omega}\right)_{j} \boldsymbol{p}_{i, k} & ; q_{j} \in \boldsymbol{q}^{i-1}, q_{k} \in \boldsymbol{q}_{i} \\ 0 & ; q_{j} \in \boldsymbol{q}_{i}, q_{k} \in \boldsymbol{q}^{i-1} \\ \mathbf{R}_{i, k}^{T} \boldsymbol{p}_{i, j}+\mathbf{R}_{i}^{T} \boldsymbol{p}_{i, j, k} & ;\left\{q_{j}, q_{k}\right\} \in \boldsymbol{q}_{i}\end{cases}
$$

The derivation is given in Appendix C.5.

This completes the recursive derivation of spatial displacements, Jacobians, and Hessians. Utilizing these recursive relationships, element-wise calculations of $\mathbf{M}_{i}$, partial derivatives of $\mathbf{M}_{i}$ for computing $\mathbf{C}_{i}$, and $\boldsymbol{G}_{i}$ are presented next.

\subsection{Recursive Computation of $\mathrm{M}_{i}^{\omega}$ and the Partial Derivatives}

First the $\mathbf{M}_{i}^{\omega}$ and the partial derivatives are derived followed by $\mathbf{M}_{i}^{v}$ and the partial derivatives.

From the definition of $\mathbf{M}_{i}^{\omega}$ given by Equation (22) and $\mathcal{M}_{i}^{\omega}$ defined in Section 2.4.2, consider any $\left[\mathbf{M}_{i}^{\omega}\right]_{j k}$ given by 


$$
\begin{aligned}
{\left[\mathbf{M}_{i}^{\omega}\right]_{j k} } & =\int_{\xi_{i}}\left\{\left(\mathbf{J}_{i}^{\omega}\right)_{j}^{T} \mathcal{M}_{i}^{\omega}\left(\mathbf{J}_{i}^{\omega}\right)_{k}\right\} \\
& =I_{x x} \int_{\xi_{i}}\left\{\left(\mathbf{J}_{i}^{\omega}\right)_{j}^{T} \operatorname{Diag}(1,1,2)\left(\mathbf{J}_{i}^{\omega}\right)_{k}\right\}
\end{aligned}
$$

The Algebraic Identity $\mathbf{1}$ is used to simplify Equation (34).

\section{Algebraic Identity 1:}

If $\mathbf{A} \in \mathbb{R}^{3 \times 3}$ is skew symmetric matrix and $B=\mathbf{A}^{\vee}$, then $\boldsymbol{B}^{T} \operatorname{Diag}(1,1,2) \boldsymbol{B}=\mathbb{T}_{2}\left(\mathbf{A}^{T} \mathbf{A}\right)$.

The proof is given in Appendix D.1.

The assumption AS5 on constant $r_{i}$ of continuum section (or any constant, symmetric shape), listed in Section 2.3 enables this algebraic simplification. Now applying Algebraic Identity 1, Equation (34) becomes

$$
\left[\mathbf{M}_{i}^{\omega}\right]_{j k}=I_{x x} \int_{\xi_{i}} \mathbb{T}_{2}\left\{\left(\mathbf{J}_{i}^{\Omega}\right)_{j}^{T}\left(\mathbf{J}_{i}^{\Omega}\right)_{k}\right\}
$$

Considering the case-wise definition of $\left(\mathbf{J}_{i}^{\Omega}\right)_{j}$ given in Equation (30) and the symmetry of $\mathbf{M}_{i}^{\omega}$, three unique cases for $\left[\mathbf{M}_{i}^{\omega}\right]_{j k}$ can be identified when; (1) $\left\{q_{j}, q_{k}\right\} \in \boldsymbol{q}^{i-1}$, (2) $q_{j} \in \boldsymbol{q}^{i-1}, q_{k} \in \boldsymbol{q}_{i}$, and (3) $\left\{q_{j}, q_{k}\right\} \in \boldsymbol{q}_{i}$. Hence, the development $\left[\mathbf{M}_{i}^{\omega}\right]_{j k}$ is presented under these three separate cases where their partial derivatives are presented under two sub cases.

Case 1: $\left\{q_{j}, q_{k}\right\} \in \boldsymbol{q}^{i-1}$

The corresponding Jacobian columns from Equation (30) are substituted into Equation (35) to obtain

$$
\left[\mathbf{M}_{i}^{\omega}\right]_{j k}=I_{x x} \int_{\xi_{i}} \mathbb{T}_{2}\left(\mathbf{R}_{i}^{T}\left\{\left(\mathbf{J}_{i-1}^{\Omega}\right)_{j}^{T}\left(\mathbf{J}_{i-1}^{\Omega}\right)_{k}\right\} \mathbf{R}_{i}\right)
$$

In the integrand of Equation (36), only $\mathbf{R}_{i}$ contains the variable of integration, $\xi_{i}$, but there are matrix terms in between the integrable $\mathbf{R}_{i}$ terms. Given the complexity of the expression, computer algebra systems run into problems when attempting to symbolically evaluate Equation (36) as is. Also, evaluating all the integral terms numerically during simulations entails a heavy computational burden. However by using suitable algebraic identities to bring the terms containing $\xi_{i}$ together, the integral can be precomputed in symbolic form prior to starting the simulation. Once the integrals are precomputed the simulation execution time significantly decreases. A detailed account on this is provided in Section 6.2. In order to simplify Equation (36) the following Algebraic Identity 2 is employed.

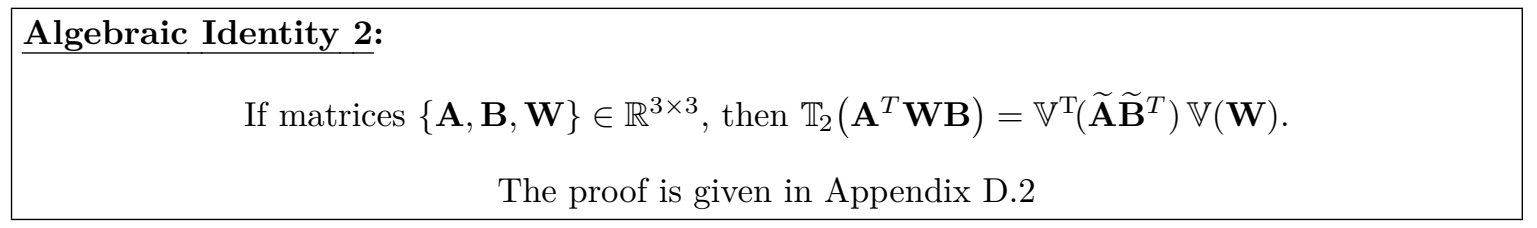

Applying Algebraic Identity 2 on Equation (36) becomes

$$
\left[\mathbf{M}_{i}^{\omega}\right]_{j k}=I_{x x} \int_{\xi_{i}} \mathbb{V}^{\mathrm{T}}\left\{\left(\widetilde{\mathbf{R}}_{i} \widetilde{\mathbf{R}}_{i}^{T}\right)\right\} \mathbb{V}\left\{\left(\mathbf{J}_{i-1}^{\Omega}\right)_{j}^{T}\left(\mathbf{J}_{i-1}^{\Omega}\right)_{k}\right\}
$$

But since $\widetilde{\mathbf{R}}_{i} \widetilde{\mathbf{R}}_{i}^{T}=\widetilde{\mathbf{I}}_{3}$, Equation (37) reduces to

$$
\left[\mathbf{M}_{i}^{\omega}\right]_{j k}=I_{x x} \int_{\xi_{i}} \mathbb{V}^{\mathrm{T}}\left(\widetilde{\mathbf{I}}_{3}\right) \mathbb{V}\left\{\left(\mathbf{J}_{i-1}^{\Omega}\right)_{j}^{T}\left(\mathbf{J}_{i-1}^{\Omega}\right)_{k}\right\}
$$

where $\left(\mathbf{J}_{i-1}^{\Omega}\right)_{j}^{T}\left(\mathbf{J}_{i-1}^{\Omega}\right)_{k}$ and $\widetilde{\mathbf{I}}_{3}$ do not depend on $\xi_{i}$. So the integral is easy to compute since $\int_{\xi_{i}}=$ $\int_{0}^{1} d \xi_{i}=1$. Also, $\mathbb{V}^{\mathrm{T}}\left(\widetilde{\mathbf{I}}_{3}\right) \mathbb{V}(\mathbf{W})=\mathbb{T}_{2}(\mathbf{W})$ where $\mathbf{W} \in \mathbb{R}^{3 \times 3}$. Therefore Equation (38) simplifies to 


$$
\left[\mathbf{M}_{i}^{\omega}\right]_{j k}=I_{x x} \mathbb{T}_{2}\left\{\left(\mathbf{J}_{i-1}^{\Omega}\right)_{j}^{T}\left(\mathbf{J}_{i-1}^{\Omega}\right)_{k}\right\}
$$

The partial derivatives of $\left[\mathbf{M}_{i}^{\omega}\right]_{j k}$ are also derived case-wise depending if $q_{s} \in \boldsymbol{q}^{i-1}$ or $q_{s} \in \boldsymbol{q}_{i}$ as

$$
\left[\mathbf{M}_{i}^{\omega}\right]_{j k, s}= \begin{cases}I_{x x} \mathbb{T}_{2}\left\{\left(\mathbf{H}_{i-1}^{\Omega}\right)_{j s}^{T}\left(\mathbf{J}_{i-1}^{\Omega}\right)_{k}+\left(\mathbf{J}_{i-1}^{\Omega}\right)_{j}^{T}\left(\mathbf{H}_{i-1}^{\Omega}\right)_{k s}\right\} & ; q_{s} \in \boldsymbol{q}^{i-1} \\ 0 & ; q_{s} \in \boldsymbol{q}_{i}\end{cases}
$$

This process is applied to all the cases of $\left[\mathbf{M}_{i}\right]_{j k}$ and $\left[\mathbf{M}_{i}\right]_{j k, s}$.

Case 2: $q_{j} \in \boldsymbol{q}^{i-1}$ and $q_{k} \in \boldsymbol{q}_{i}$

Substituting the relevant Jacobian columns from Equation (30) into Equation (35) and applying Algebraic Identity 2 yield

$$
\left[\mathbf{M}_{i}^{\omega}\right]_{j k}=I_{x x} \mathbb{V}^{\mathrm{T}}\left\{\int_{\xi_{i}}\left(\widetilde{\mathbf{R}}_{i} \widetilde{\mathbf{R}}_{i, k}^{T}\right)\right\} \mathbb{V}\left\{\left(\mathbf{J}_{i-1}^{\Omega}\right)_{j}^{T}\right\}
$$

Note that, the integration is only applied to $\left(\widetilde{\mathbf{R}}_{i} \widetilde{\mathbf{R}}_{i, k}^{T}\right)$ because $\left(\mathbf{J}_{i-1}^{\Omega}\right)_{j}$ is independent of $\xi_{i}$. This is always a valid operation because the vectorization operation is not affected by the integration nor it affects the integration.

And taking the partial derivatives of Equation (41), $\left[\mathbf{M}_{i}^{\omega}\right]_{j k, s}$ is given by

$$
\left[\mathbf{M}_{i}^{\omega}\right]_{j k, s}= \begin{cases}I_{x x} \mathbb{V}^{\mathrm{T}}\left\{\int_{\xi_{i}}\left(\widetilde{\mathbf{R}}_{i} \widetilde{\mathbf{R}}_{i, k}^{T}\right)\right\} \mathbb{V}\left\{\left(\mathbf{H}_{i-1}^{\Omega}\right)_{j s}^{T}\right\} & ; q_{s} \in \boldsymbol{q}^{i-1} \\ I_{x x} \mathbb{V}^{\mathrm{T}}\left\{\int_{\xi_{i}}\left(\widetilde{\mathbf{R}}_{i} \widetilde{\mathbf{R}}_{i, k}^{T}\right)_{, s}\right\} \mathbb{V}\left\{\left(\mathbf{J}_{i-1}^{\Omega}\right)_{j}^{T}\right\} & ; q_{s} \in \boldsymbol{q}_{i}\end{cases}
$$

Case 3: $\left\{q_{j}, q_{k}\right\} \in \boldsymbol{q}_{i}$

Likewise, substitute the relevant columns from Equation (30) into Equation (35). Here the Algebraic Identity $\mathbf{2}$ is not required as there are no terms in between the integrable terms.

$$
\left[\mathbf{M}_{i}^{\omega}\right]_{j k}=I_{x x} \mathbb{T}_{2}\left(\int_{\xi_{i}} \mathbf{R}_{i, j}^{T} \mathbf{R}_{i, k}\right)
$$

And similarly $\left[\mathbf{M}_{i}^{\omega}\right]_{j k, s}$ is given by

$$
\left[\mathbf{M}_{i}^{\omega}\right]_{j k, s}= \begin{cases}0 & ; q_{s} \in \boldsymbol{q}^{i-1} \\ I_{x x} \mathbb{T}_{2}\left(\int_{\xi_{i}} \mathbf{R}_{i, j}^{T} \mathbf{R}_{i, k}\right)_{, s} & ; q_{s} \in \boldsymbol{q}_{i}\end{cases}
$$

\subsection{Recursive Computation of $\mathrm{M}_{i}^{v}$ and the Partial Derivatives}

Similarly, substituting $\mathcal{M}_{i}^{v}=m_{i} \mathbf{I}_{3}$ defined in Section 2.4.2 into Equation (23), the $\left[\mathbf{M}_{i}^{v}\right]_{j k}$ is given by

$$
\left[\mathbf{M}_{i}^{v}\right]_{j k}=m_{i} \int_{\xi_{i}}\left\{\left(\mathbf{J}_{i}^{v}\right)_{j}^{T}\left(\mathbf{J}_{i}^{v}\right)_{k}\right\}
$$

Here also, the evaluation of $\left[\mathbf{M}_{i}^{v}\right]_{j k}$ is presented case-wise similar to Section 3.2. 
Case 1: $\left\{q_{j}, q_{k}\right\} \in \boldsymbol{q}^{i-1}$

The respective cases of Equation (31) are substituted into Equation (45) and expanded as

$$
\begin{aligned}
{\left[\mathbf{M}_{i}^{v}\right]_{j k}=m_{i}\left(\mathbf{J}_{i-1}^{v}\right)_{j}^{T}\left\{\left(\mathbf{J}_{i-1}^{v}\right)_{k}+\left(\mathbf{J}_{i-1}^{\Omega}\right)_{k}\left(\int_{\xi_{i}} \boldsymbol{p}_{i}\right)\right\}+m_{i}\left(\int_{\xi_{i}} \boldsymbol{p}_{i}^{T}\right)\left(\mathbf{J}_{i-1}^{\Omega}\right)_{j}^{T}\left(\mathbf{J}_{i-1}^{v}\right)_{k} } & \\
& +m_{i} \int_{\xi_{i}}\left\{\boldsymbol{p}_{i}^{T}\left(\mathbf{J}_{i-1}^{\Omega}\right)_{j}^{T}\left(\mathbf{J}_{i-1}^{\Omega}\right)_{k} \boldsymbol{p}_{i}\right\}
\end{aligned}
$$

Here also, the integrable components (that are functions of $\xi_{i}$ ) of the last term of Equation (46) are separated by Jacobian terms. Analogous to Equation (36), the integrable components are brought together by employing the following Algebraic Identity 3.

\section{Algebraic Identity 3:}

$$
\text { If }\{\boldsymbol{A}, \boldsymbol{B}\} \in \mathbb{R}^{3 \times 1} \text { and } \mathbf{Q} \in \mathbb{R}^{3 \times 3} \text {, then } \boldsymbol{A}^{T} \mathbf{Q} \boldsymbol{B}=\mathbb{V}^{\mathrm{T}}\left(\boldsymbol{A} \boldsymbol{B}^{T}\right) \mathbb{V}(\mathbf{Q}) \text {. }
$$

The proof is given in Appendix D.3

Therefore, Equation (46) is rewritten as

$$
\begin{aligned}
{\left[\mathbf{M}_{i}^{v}\right]_{j k}=m_{i}\left(\mathbf{J}_{i-1}^{v}\right)_{j}^{T}\left\{\left(\mathbf{J}_{i-1}^{v}\right)_{k}+\left(\mathbf{J}_{i-1}^{\Omega}\right)_{k}\left(\int_{\xi_{i}} \boldsymbol{p}_{i}\right)\right\} } & +m_{i}\left(\int_{\xi_{i}} \boldsymbol{p}_{i}^{T}\right)\left(\mathbf{J}_{i-1}^{\Omega}\right)_{j}^{T}\left(\mathbf{J}_{i-1}^{v}\right)_{k} \\
& +m_{i} \mathbb{V}^{\mathrm{T}}\left\{\int_{\xi_{i}}\left(\boldsymbol{p}_{i} \boldsymbol{p}_{i}^{T}\right)\right\} \mathbb{V}\left\{\left(\mathbf{J}_{i-1}^{\Omega}\right)_{j}^{T}\left(\mathbf{J}_{i-1}^{\Omega}\right)_{k}\right\}
\end{aligned}
$$

Notice that, since integrable terms of the last term of Equation (47) are now together, the integral can be symbolically solved without affecting the result prior to starting the simulation.

And now partial derivatives of Equation (47) are taken to compute relevant $\left[\mathbf{M}_{i}^{v}\right]_{j k, s}$ as

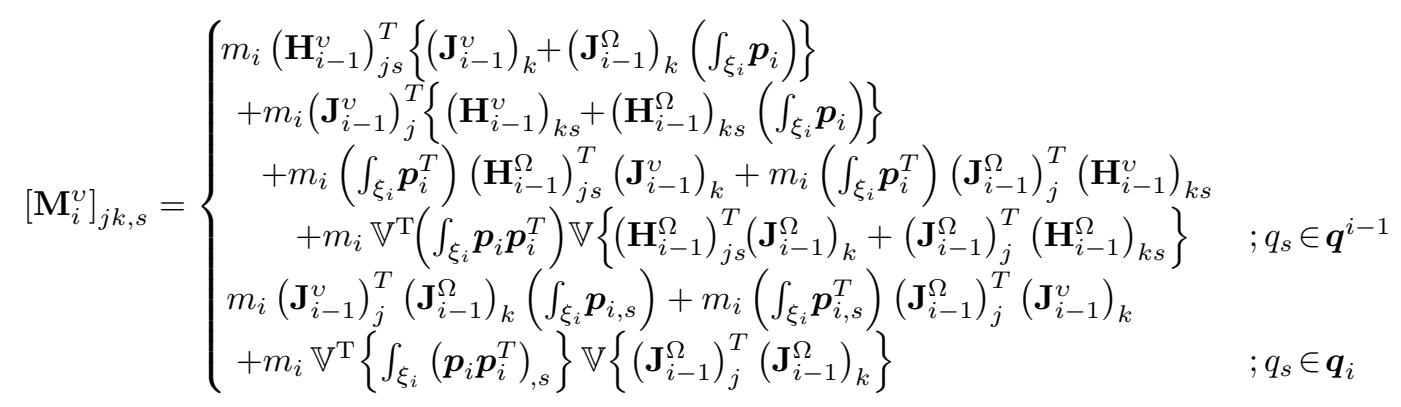

Case 2: $q_{j} \in \boldsymbol{q}^{i-1}$ and $q_{k} \in \boldsymbol{q}_{i}$

Similarly, the corresponding cases of Equation (31) are substituted into Equation (45) and applying Algebraic Identity 3 to obtain

$$
\left[\mathbf{M}_{i}^{v}\right]_{j k}=m_{i}\left(\mathbf{J}_{i-1}^{v}\right)_{j}^{T}\left(\int_{\xi_{i}} \boldsymbol{p}_{i, k}\right)+m_{i} \mathbb{V}^{\mathrm{T}}\left\{\int_{\xi_{i}}\left(\boldsymbol{p}_{i} \boldsymbol{p}_{i, k}^{T}\right)\right\} \mathbb{V}\left\{\left(\mathbf{J}_{i-1}^{\Omega}\right)_{j}^{T}\right\}
$$

Now the related $\left[\mathbf{M}_{i}^{v}\right]_{j k, s}$ is computed as

$$
\left[\mathbf{M}_{i}^{v}\right]_{j k, s}= \begin{cases}m_{i}\left(\mathbf{H}_{i-1}^{v}\right)_{j}^{T}\left(\int_{\xi_{i}} \boldsymbol{p}_{i, k}\right)+m_{i} \mathbb{V}^{\mathrm{T}}\left(\int_{\xi_{i}} \boldsymbol{p}_{i} \boldsymbol{p}_{i, k}^{T}\right) \mathbb{V}\left\{\left(\mathbf{H}_{i-1}^{\Omega}\right)_{j}^{T}\right\} & ; q_{s} \in \boldsymbol{q}^{i-1} \\ m_{i}\left(\mathbf{J}_{i-1}^{v}\right)_{j}^{T}\left(\int_{\xi_{i}} \boldsymbol{p}_{i, k, s}\right)+m_{i} \mathbb{V}^{\mathrm{T}}\left\{\int_{\xi_{i}}\left(\boldsymbol{p}_{i} \boldsymbol{p}_{i, k}^{T}\right)_{, s}\right\} \mathbb{V}\left\{\left(\mathbf{J}_{i-1}^{\Omega}\right)_{j}^{T}\right\} & ; q_{s} \in \boldsymbol{q}_{i}\end{cases}
$$

Case 3: $\left\{q_{j}, q_{k}\right\} \in \boldsymbol{q}_{i}$

Finally, the relevant cases of Equation (31) are substituted into Equation (45) to obtain 


$$
\left[\mathbf{M}_{i}^{v}\right]_{j k}=m_{i} \int_{\xi_{i}}\left(\boldsymbol{p}_{i, j}^{T} \boldsymbol{p}_{i, k}\right)
$$

Similarly, corresponding $\left[\mathbf{M}_{i}^{v}\right]_{j k, s}$ is calculated as

$$
\left[\mathbf{M}_{i}^{v}\right]_{j k, s}= \begin{cases}0 & ; q_{s} \in \boldsymbol{q}^{i} \\ m_{i}\left(\boldsymbol{p}_{i, j}^{T} \boldsymbol{p}_{i, k}\right)_{, s} & ; q_{s} \in \boldsymbol{q}_{i}\end{cases}
$$

This completes the derivation of elements of $\mathbf{M}_{i}$ and their partial derivatives required to compute $\mathbf{C}_{i}$.

\subsection{Recursive Computation of $\boldsymbol{G}_{i}$}

From the definition in Equation $(25),\left[\boldsymbol{G}_{i}^{g}\right]_{j}$ is given by

$$
\left[\boldsymbol{G}_{i}^{g}\right]_{j}=m_{i}\left(\int_{\xi_{i}} \Psi_{i, j}^{T}\right) \boldsymbol{g}
$$

Since $\Theta \Theta^{T}=\mathbf{I}_{3}$, without affecting the outcome, Equation (53) is rearranged and substituted $\left(\mathbf{J}_{i}^{v}\right)_{j}$ from Equation (19) to obtain

$$
\begin{aligned}
{\left[\boldsymbol{G}_{i}^{g}\right]_{j} } & =m_{i} \int_{\xi_{i}}\left\{\Psi_{i, j}\left(\Theta_{i} \Theta_{i}^{T}\right)\right\} \boldsymbol{g} \\
& =m_{i} \int_{\xi_{i}}\left\{\left(\Theta_{i}^{T} \Psi_{i, j}\right)^{T} \Theta_{i}^{T}\right\} \boldsymbol{g} \\
& =m_{i} \int_{\xi_{i}}\left(\mathbf{J}_{i}^{v}\right)_{j}^{T} \Theta_{i}^{T} \boldsymbol{g}
\end{aligned}
$$

Substituting the case-wise definition of $\left(\mathbf{J}_{i}^{v}\right)_{j}$ from Equation (31), Equation (54) is expressed recursively as

$$
\left[\boldsymbol{G}_{i}^{g}\right]_{j}= \begin{cases}m_{i}\left\{\left(\mathbf{J}_{i-1}^{v}\right)_{j}+\left(\mathbf{J}_{i-1}^{\Omega}\right)_{j}\left(\int_{\xi_{i}} \boldsymbol{p}_{i}\right)\right\}^{T} \Theta_{i-1}^{T} \boldsymbol{g} & ; q_{j} \in \boldsymbol{q}^{i-1} \\ m_{i}\left(\int_{\xi_{i}} \boldsymbol{p}_{i, j}^{T}\right) \Theta_{i-1}^{T} \boldsymbol{g} & ; q_{j} \in \boldsymbol{q}_{i}\end{cases}
$$

The elastic PE of continuum sections are independent of position or orientation and hence can be directly accounted for as

$$
\boldsymbol{G}_{i}^{e}=\mathbf{K}_{i}^{e} \boldsymbol{q}_{i}
$$

This completes the derivation of $\boldsymbol{G}_{i}$. These recursive results are then substituted in Equations (8), (12), and (16) to compute $\mathbf{M}, \mathbf{C}$, and $\boldsymbol{G}$ of the complete EoM given by Equation (3). For the ease of reference, Algorithm 1 summarizes the application of mathematical results derived in this section for formulating the EoM.

\section{Prototype Arm and the Simulation Model}

\subsection{Details of the PMA Actuated Prototype Continuum Arm}

Each continuum section of the prototype arm (shown in Figure 1c) consists of three mechanically identical extending PMA's (Caldwell et al., 1995; Godage et al., 2012b) with $L_{i 0}=0.15 \mathrm{~m}, l_{i: \min }=0 \mathrm{~m}$, and $l_{i: \max }=0.065 \mathrm{~m}$. These actuators are pressurized in the range $(0-6 \mathrm{bars})$. Silicone rubber tubes of inner diameter $(I D) 7.5 \mathrm{~mm}$ and outer diameter $(O D) 9.5 \mathrm{~mm}$ were used as the PMA containment layer. The outer braided sheath is made from polyester $(\min . O D=7 \mathrm{~mm}$, max. $O D=17 \mathrm{~mm}$ ) and forms the core platform for the motion of the actuator. Nylon union tube connectors $(I D=4 \mathrm{~mm})$ seal the Silicone tubes and control the air in/out flow. Rigid plastic frames (ABS thermoplastic) of $r_{i}=0.0125 \mathrm{~m}$ and $2.54 \mathrm{~mm}$ thickness (see Figure 4a) are used to mount the PMA's and connect adjacent continuum 


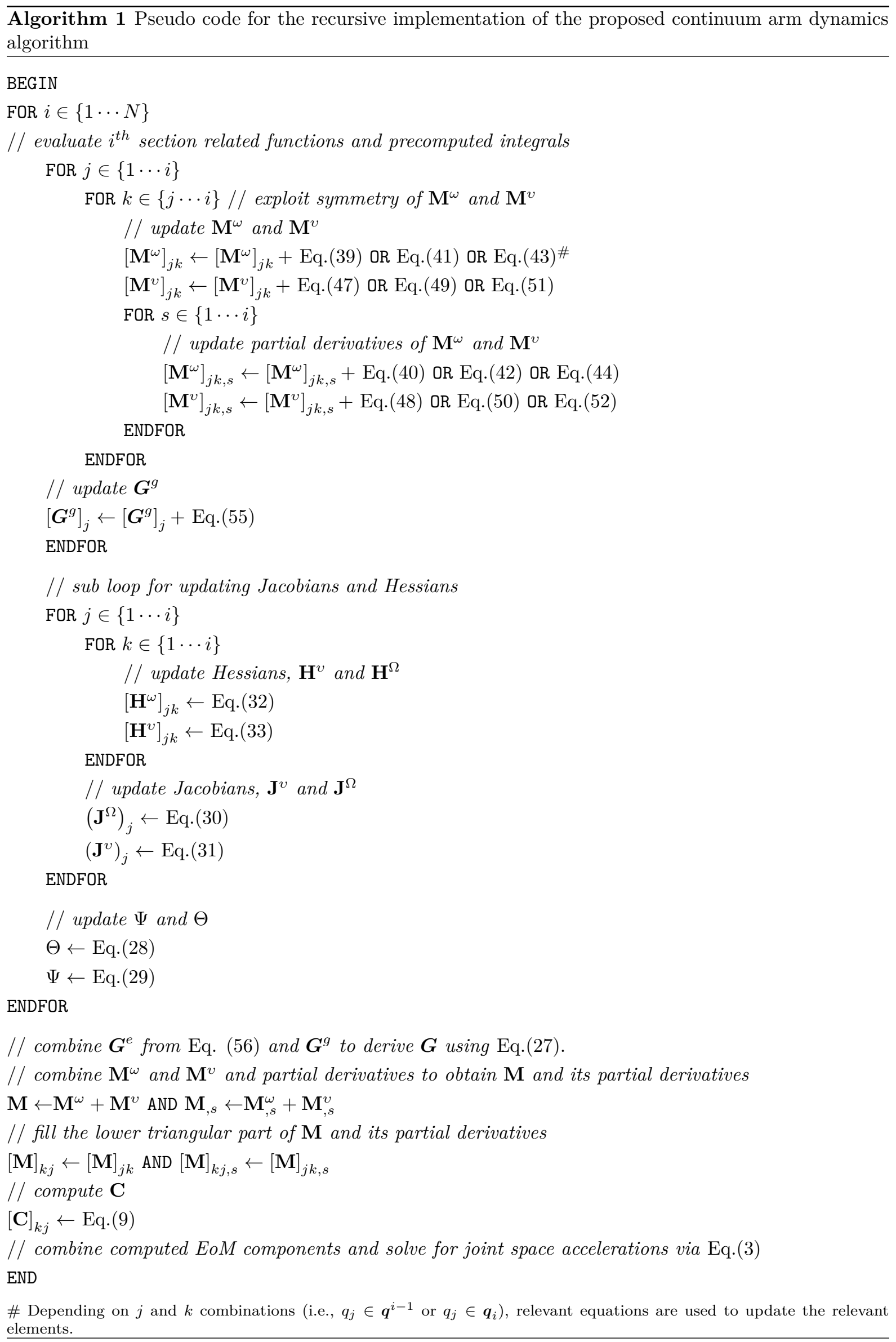




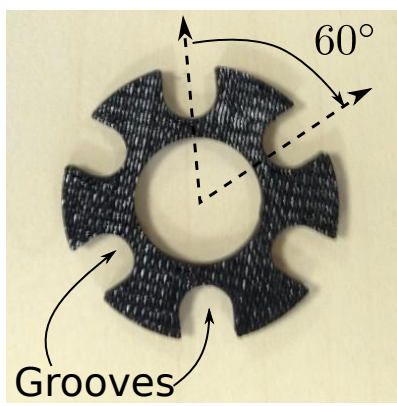

(a)

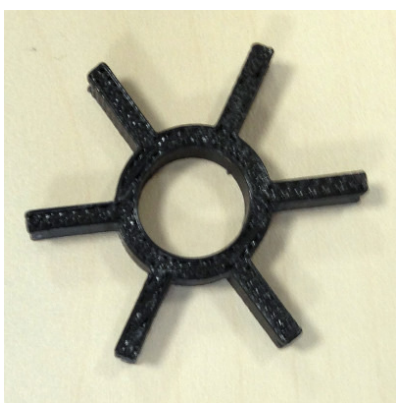

(b)

Figure 4: (a) 3D printed rigid plastic mount frames $(2.54 \mathrm{~mm}$ thickness) of continuum sections where the grooves are designed to fit the union tube connector dimensions. The adjacent continuum sections are afixed at a $\frac{\pi}{3}$ orientation offset as shown. (b) 3D printed plastic constrainers $(2.54 \mathrm{~mm}$ thickness). Three groves house the PMA's and the rest route the pressure supplying tubes.

sections with a $\frac{\pi}{3}$ rad angle offset about the $Z$ axis of the arm tip coordinate frame. This offset ensures easy mounting of the PMA's and pressurized air supply tubes. Rigid plastic constrainers (Figure 4b) maintain the PMA's parallel to the neutral axis with a clearance $\left(\frac{2 \pi}{3} \mathrm{rad}\right.$ apart at $\left.r_{i}\right)$ from each other. These constrainers also provide improved torsional stiffness minimizing the possible deviations from the assumed circular shapes when operating out of the plane under the influence of gravity. Each continuum section, inclusive of the tubing and constrainers has an approximate mass of $0.13 \mathrm{~kg}$. The gravitational acceleration used in the simulations is $9.81 \mathrm{~ms}^{-2}$.

\subsection{Validity of the Model Assumptions}

The assumptions made in Section 2.3 have significant implications to the final dynamic model. Therefore, to validate the model, the prototype arm shown in Figure 1c needs to comply with those assumptions. This section discusses how the prototype arm adequately complies with the assumptions for the experiments carried out in Section 5 .

The modal kinematics, on which the proposed dynamic model derived upon, assume circular arc shaped deformation in each continuum section. Assumption AS1 arises from this claim. However, under the influence of gravity, preceding sections are subjected to external forces/moments when subsequent sections bend in multiple planes. This can cause sections to deviate from the assumed circular arc. The prototype arm mitigates this problem through the use of evenly distributed constrainers fitted along the length of continuum sections. This approach significantly increase the torsional stiffness of the arm to torsionally "lock" the PMA's to prevent twisting about its neutral axis. Otherwise, the continuum sections may deform in helical shape. In addition, the high elastic stiffness (relative to the gravity loading) of the Silicon reduces deviations from the circular arc. Figure 5 compares a static pose of the prototype arm with the kinematic model given by Equation (57). From this figure it can be seen that there is an excellent fit between the physical system and the model and this helps to validate the assumption.

The prototype arm is powered by extending PMA's which also provide the structural support without the need for a backbone found in many continuum systems. Thus satisfies assumption AS2. The prototype arm sections are tightly bundled and constrained cylindrically to comply with assumption AS3. Also, the prototype fulfills assumption AS4 because the PMA's are firmly attached to the relevant individual sections and therefore the length variations do not kinematically affect the deformation of other sections. The construction of the PMA's make use of Polyester braided sheath which (except under extreme pressure) prevents it expanding radially and drive the axial expansion. Also, the constrainers along the length maintains the clearance of the PMA's during operation thus meeting assumption AS5. The amount of air present in each continuum section can be safely neglected and hence the mass of each section is only due to the construction materials. Further, the PMA's, pressure supplying tubes and constrainers are uniformly distributed along the length of continuum sections. The union connectors $\left(\right.$ Nylon $1300 \mathrm{kgm}^{-3}$ ) and mounting frames (ABS thermoplastic $1070 \mathrm{kgm}^{-3}$ ) at either end of the sections have lower densities than that of the PMA's (Silicone $2300 \mathrm{kgm}^{-3}$ ). This gives essentially a uniform linear density and assumption AS6 holds. Therefore, the dynamic model given in Equation (58) can be applied to the prototype continuum arm. 


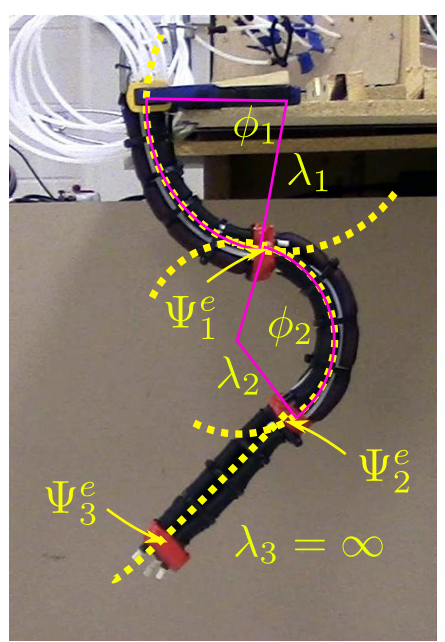

(a)

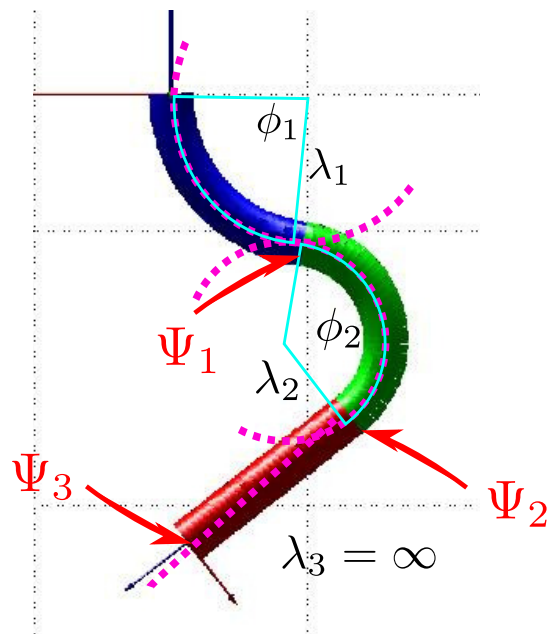

(b)

Figure 5: Comparison of modal kinematic result against the prototype multisection continuum arm (a) The prototype arm with curvature radii overlaid for base, mid, and distal continuum sections: $\left\{\lambda_{1}=110.4 \mathrm{~mm}, \phi_{1}=1.44 \mathrm{rads}\right\}$, $\left\{\lambda_{1}=70.2 \mathrm{~mm}, \phi_{1}=2.35 \mathrm{rads}\right\}$, and $\left\{\lambda_{3}=\infty, \phi_{3}=0\right\}$ for joint space variables $\boldsymbol{q}_{1}=[0.027,0,0]^{T}, \boldsymbol{q}_{2}=[0,0.044,0]^{T}$, and $\boldsymbol{q}_{3}=[0,0,0]^{T}$. (b) Forward kinematic result for the same joint space variables inputs employed in Figure 5a with curvature radii overlaid. Result closely matches holding the circular arc shape assumption AS1 made in Section 2.3 . The section tip positions of base, mid, and distal sections of the prototype arm are denoted by $\Psi_{1}^{e}$, $\Psi_{2}^{e}$, and $\Psi_{3}^{e}$. Corresponding kinematic model's tip positions are denoted by $\Psi_{1}, \Psi_{2}$, and $\Psi_{3}$ respectively.

\subsection{Prototype Continuum Arm Simulation Model}

The physical parameter values employed in subsequent simulations were taken from the prototype arm and are listed in Section 4.1. The prototype arm continuum sections bend close to $200^{\circ}$ within the PMA operation range. As noted in Section 2.2, the modal dynamics is based on the modal kinematics, which is essentially a high dimensional approximation technique. For the approximation used in this paper, an absolute position error of $0.005 \mathrm{~m}$ ( $1 \%$ of the total length when normalized to the unactuated length, $\sum_{i=1}^{3} L_{i 0}=0.474 m$ ) at the arm tip was considered acceptable. Therefore order 11 expansion for all MHTM elements were chosen which resulted $0.0043 \mathrm{~m}$ absolute maximum position error (A detailed account on the accuracy of modal approach is provided in Section 6.1). Substituting the parameter values listed in Section 4.1, the complete MTHM, $\mathbf{T}^{3} \in S E(3)$ from Equation (2) is derived as

$$
\mathbf{T}^{3}(\boldsymbol{\xi}, \boldsymbol{q})=\prod_{k=1}^{N=3}\left\{\mathbf{T}_{k}\left(\boldsymbol{q}_{k}, \xi_{k}\right) \mathbf{T}_{k}^{J}\right\}
$$

where the constant $\mathbf{T}_{k}^{J} \in S E$ (3) accounts for the $\frac{\pi}{3}$ angle offset and the $0.008 \mathrm{~m}$ displacement offset along the $Z^{\prime}$ axis (due to the required union tube connector clearance to mount) between adjacent continuum sections.

The prototype arm continuum sections do not have a deformable backbones and the bending is due to the linear elongation of PMA's. The elastic energy is therefore results chiefly from the extension of the Silicon bladders of PMA's. The bending PE of individual PMA's are negligible compared to the elastic PE as shown in (Godage et al., 2012b). One of the limitations of previous continuum arm models was the lack of the mechanical limits of variable length actuators. As a result many produced solutions beyond the physical robot's realizable task-space (Godage et al., 2012b). By defining stiffness coefficients as functions of PMA length variation of, the mechanical limits can be emulated. This is achieved by assigning a high stiffness value, $K_{i: \max }=10^{6} \mathrm{Nm}^{-1}$, beyond maximum and minimum length variations and the actual stiffness values, $K_{i: m i n}$, within the operational range as a function of PMA length variation. A smooth approximation ensures rapid simulations without discontinuities avoiding high frequency under-damped vibratory outputs. The stiffness variation used in simulations is given by

$$
K_{i j}^{e}=K_{i: \min }^{e}+\frac{1}{2} K_{\text {max }}^{e}\left[2+\tanh \left\{\mu\left(l_{i j}-l_{\text {max }}\right)\right\}-\tanh \left\{\mu\left(l_{i j}-l_{\text {min }}\right)\right\}\right], \quad \forall\{i, j\} \in\{1,2,3\}
$$


where $\mu=2000$ specifies the rate of stiffness variation at the limits.

These elastic stiffness limiting values and damping coefficients were approximately identified by following an experimental procedure similar to the method proposed in (Godage et al., 2012b). The results from (Godage et al., 2012b) provide estimate stiffness and damping coefficients for variable length continuum sections. However, once continuum sections serially connected and highly constrained (to eliminate torsion) these parameters no longer hold. This is due to the added weight of subsequent continuum sections as seen by preceding sections and higher inter-PMA friction within highly constrained continuum sections.

Because of these reasons, accurate modeling of PMA's solely based on mechanical parameters is infeasible. Therefore, step response of each PMA of any $i^{t h}$ continuum section is analyzed and utilized to experimentally identify the section's elastic stiffness $\left(\mathbf{K}_{i}^{e}\right)$ and damping $\left(\mathbf{D}_{i}\right)$ parameters. Each PMA of a section is provided with a step input of 5 bars and the input pressure profile and dynamic response are recorded. The dynamic responses are obtained in the task-space as the relevant continuum section's tip trajectory by a two-camera setup explained in Section 5.2. Then, the task-space dynamic response of the simulation model for the same step pressure input is tuned to match the experimental data by varying the respective PMA's elastic stiffness (mainly affects the steady state errors) and damping coefficients (mainly affects the transient errors). The tuning process was done by manually estimating the coefficients from starting values found in (Godage et al., 2012b). Because this is an estimation process, attempts were made to obtain good agreements between the simulated and experimental data.

The elastic stiffness and damping coefficients that produce the least transient and steady state errors were then recorded. As there are three PMA's per continuum section, the mean value of the recorded stiffness and damping coefficients are assigned as the elements of $\mathbf{K}_{i}^{e}$ and $\mathbf{D}_{i}$ for that section. This yielded $K_{1: \min }^{e}=2300 \mathrm{Nm}^{-1}, K_{2: \text { min }}^{e}=K_{3: \min }^{e}=1700 \mathrm{Nm}^{-1}$ (rounded to the nearest 100) and the damping coefficients, $D_{1}=110$ and $\left\{D_{2}, D_{3}\right\}=90$ (rounded to the nearest 10). Note that the hysteretic effects are negligible relative to the damping effects and are not considered in this experiment. Hence the EoM employed in simulations is given by

$$
\mathbf{M} \ddot{\boldsymbol{q}}+\mathbf{C} \dot{\boldsymbol{q}}+\mathbf{D} \dot{\boldsymbol{q}}+\boldsymbol{G}=\boldsymbol{\tau}_{e}
$$

where $\mathbf{D}=\operatorname{Diag}\left\{D_{1} \eta, D_{2} \eta, D_{3} \eta\right\} \in \mathbb{R}^{9 \times 9}$ and $\eta=[1,1,1]$.

The simulations were carried out in MATLAB computer program. The MATLAB environment was chosen for its rich selection of toolboxes and ease of use. The SIMULINK platform was used with variablestep ODE15s routine for its speed in handling complex dynamic systems such as the one discussed in this paper. The simulation output was recorded at 30 data samples per second for high accuracy of results and the ease of simulation movie creation at 30 frames per second. The model is able to simulate motions across the entire task-space without restrictive starting poses enforced on previous models at singular configurations.

\subsection{Simulation Results and Scalability}

Figure 6 shows the simulation results of two multisection continuum arms employing the proposed spatial dynamic model. Figure 6a shows five instances of a dynamic simulation of the prototype continuum arm model. The model has nine DoF and the simulation was generated for random input forces on different joint space variables (i.e., PMA's). The simulation starts from a straight-arm singular pose (for which the previous curve parametric models were undefined) and exhibits omnidirectional bending in task-space. The simulation successfully demonstrates the passive compliance (in the form of decaying oscillations) in continuum arms. The extensibility and computational efficiency of the model is then presented by simulating a 10-section continuum arm in Figure 6b. The complete model has 30 DoF (3 for each section) and the simulation starts from a random starting pose and then continues to models the arm's passive dynamic behavior under the influence of gravity. The starting pose was generated by assigning random initial conditions for joint space variables of sections 1,3,6,7,10 (base of section 1 coincides with the taskspace origin) while remaining DoF are assigned 0. Hence the starting pose incorporates both bending and straight arm (undefined in previous curve parametric models) continuum sections to demonstrates the models numerical stability. The full simulations are shown in Extension 1. 


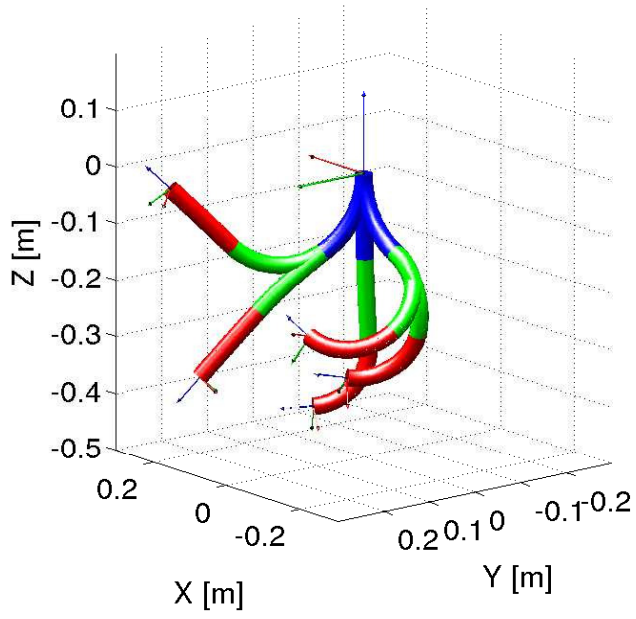

(a)

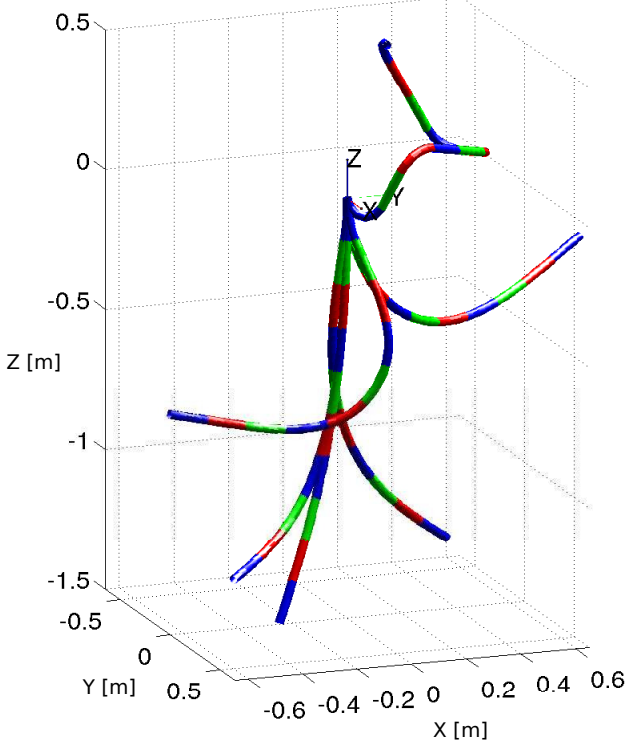

(b)

Figure 6: Dynamic spatial simulation results. (a) Snapshots of a three-section prototype continuum arm motion simulation in space, (b) Snapshots of a 10-section continuum arm motion in space. This verifies the extensibility of the proposed recursive spatial dynamic model for arbitrarily long continuum arm dynamic simulations.

\section{$5 \quad$ Experimental Results}

The objective of the following experiments is to validate the compliant dynamic responses of the PMA actuated prototype multisection continuum arm using the proposed modal dynamics. Experiments are carried out to compare the modeling accuracy of both active and passive responses of the prototype arm. The experiments are conducted in the joint-space and the results are compared in the taskspace. In the following experiments, the prototype arm pressure commands are applied at arbitrary times and the pressure profiles are recorded with temporal information. These pressure profiles are then used to generate the input pressure commands (as SIMULINK input source, structure with time, from the workspace) to the prototype arm dynamic model given by Equation (58). The experimental and simulation temporal responses are then validated by comparing each section tip trajectories in the task-space.

\subsection{Experimental Setup}

The overall experimental system block diagram is illustrated in Figure 7a. There are five main parts in the experimental setup: input pressure source, digital pressure controllers, pressure command interface, prototype arm (discussed in Section 4.1), and the task-space trajectory tracking system.

The input pressure to the setup is a constant 7bar pressure supplied by a GMC 6310 air compressor which is distributed to Pneumax ${ }^{\circledR}$ 171E2N.T.D.0009S digital proportional pressure regulators via $4 \mathrm{~mm}$ (OD) Polyurethane tubing. The pressure regulators are arranged in a compact assembly as shown in Figure 7b. PMA's of the prototype arm are connected to 9 separate pressure regulators. The pressure regulators are operated at $24 \mathrm{~V} \mathrm{DC}$ and have inbuilt digital pressure control system to output any pressure between 0-9bars with a maximum input pressure of 10bars. They also support 4800bps RS232 based digital command-response type interface that support real-time pressure commanding and reading. USBRS232 hardware interfaces are attached to pressure controllers that are connected to the commanding host via three USB hubs (each with 4 USB ports).

The pressure commands for the prototype continuum arm are generated by a computer user interface. Note that the pressure step inputs for all the experiments are rate limited to $12.5 \mathrm{bar} / \mathrm{sec}$ to reflect the gradual pressure build up observed in the PMA's. The generated pressure commands are communicated to pressure regulators via a RS232 communication line at $40 \mathrm{~Hz}$. Given the bandwidth of pneumatic valves (Godage et al., 2012b), this rate ensures high fidelity of input pressure commands. For each experiment carried out, the pressure commands are saved in separate text files with the time stamps 


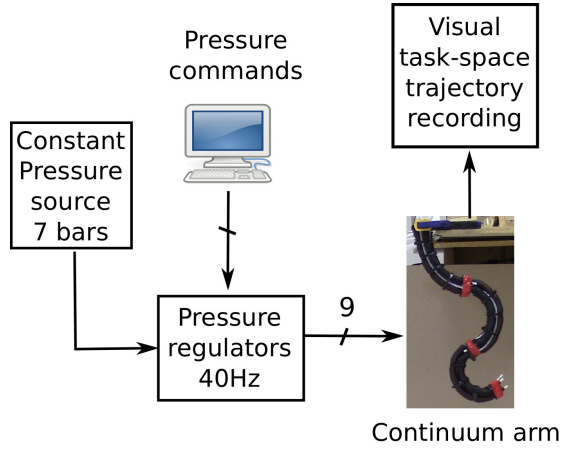

(a)

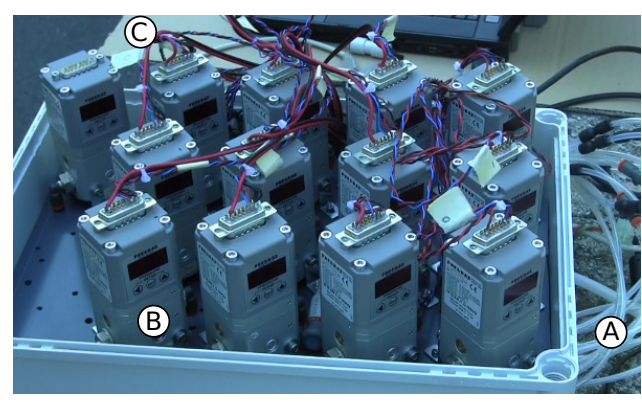

(b)

Figure 7: (a) The block diagram of the experimental setup showing the main components: pressure source, control unit, pressure control interface, continuum robot, and the visual trajectory tracking system. (b) The pressure regulator assembly: A:-The pressure distribution tubing for individual PMA's, B:-Pneumax ${ }^{\circledR}$ 171E2N.T.D.0009S digital proportional regulators, C:-Power supply and communication wiring.

and these data are then used to generate input force commands for the dynamic simulations. Trajectory tracking system is presented in detail in the following Section.

\subsection{Spatial Task-space Trajectory Tracking Through Video Reconstruction}

As noted in Section 4.2, the constrainers installed along the length of each sections effectively eliminate possible deviations from the circular arc deformation assumptions at section level. Therefore for the prototype arm considered herein, only the tip coordinates of each continuum section are recorded to determine the accuracy of the model.

For measuring the section spatial tip trajectories of each section in the task-space, a two-camera setup was used and shown in Figure 8a. It is a simple but effective system for the task at hand. Each camera (Panasonic HDC-HS700) records in HD (1920px $\times 1080 \mathrm{px}$ where px denotes pixels) definition at 60 frames per second. One camera is placed with a $-5^{\circ}$ offset to the $+\mathrm{X}$ axis (normal to $\mathrm{Y}$ axis to capture the $\mathrm{Y}$ coordinates) and the other is placed with $-5^{\circ}$ offset to the $+\mathrm{Y}$ axis (normal to $\mathrm{X}$ axis to capture the $\mathrm{X}$ coordinates) at a $8 \mathrm{ft}$ clearance (to reduce perspective errors), parallel to the center of the unactuated prototype arm. The $-5^{\circ}$ offset enables to observe the in-plane motions in both $\mathrm{X}$ and $\mathrm{Y}$ axes without occlusion.

After the experiments, the videos are clipped for the duration (start to finish), synchronized by matching the motion start times, cropped to the task-space size, and arranged side by side (Figure 8b). Then images from the videos are generated for each frame by using "ffmpeg ${ }^{\circledR}$ " software. The tracking system covered $\sim 10 \mathrm{~m}^{3}$ volume and produced $0.0014 \mathrm{~m} / \mathrm{px}$ tracking resolution within the $\sim 0.55 \mathrm{~m}^{3}$ taskspace of the prototype arm. Statistical measurements carried out in multiple occasions for the known arm base location resulted $\pm 4 \mathrm{px}$ error which translates to $\pm 0.0056 \mathrm{~m}$ in task-space coordinates. Due to it's negligible size ( $1.18 \%$ of prototype arm length) this error is considered acceptable for the experiments.

The coordinates values of each continuum section tip of the prototype $\left(\Psi_{1}^{e}, \Psi_{2}^{e}\right.$, and $\left.\Psi_{3}^{e}\right)$ in video frames are manually recorded in pixel values via image analysis in MATLAB. Figure 8b-IMGX shows an instance of the manual tip location tracking where 8b-IMGX records the $\mathrm{Z}$ and $\mathrm{Y}$ coordinates and 8b-IMGY records $\mathrm{Z}$ and $\mathrm{X}$ coordinates relative to the corresponding base coordinates (marked BX and BY). Image processing techniques were not used because of occlusion of markers. Also, the tracking bands are attached on the periphery of the arm but the tip location is located at the center of each band. Thus, manual tracking proved straightforward and effective for the purpose of this paper with $1.18 \%$ normalized error. Figure 8b-IMGY shows how the tip locations were deduced from the different marker band orientations.

The data recorded in pixel values are then scaled to task-space coordinates. Scaling factors for each perspective of Figure $8 \mathrm{~b}$ is computed by finding the known unactuated arm length $(0.474 \mathrm{~m})$ in pixel values which provide the $m / p x$ scale. Task-space trajectories are then constructed from the X,Y,Z coordinates. Finally, data is re-sampled at 10 frames per second (utilizing MATLAB's "interp1" sub-routine with "PCHIP" interpolation method) for ease of comparison on plots. The re-sampling process also averages out any user introduced errors during manual data recording. 


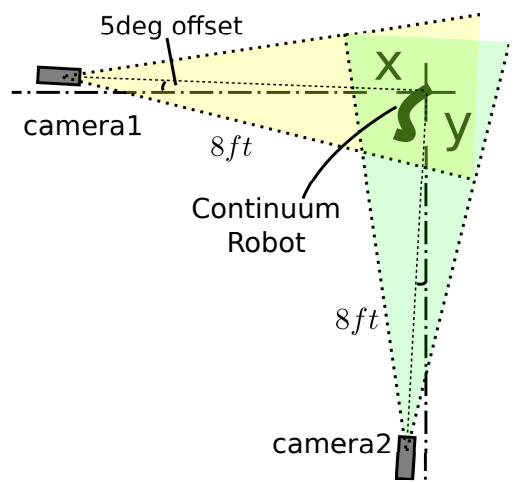

(a)

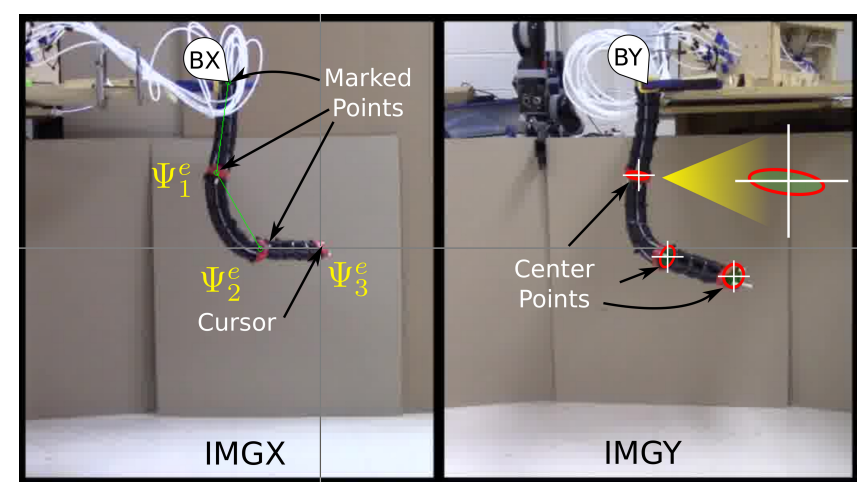

(b)

Figure 8: (a) The dorsal view schematic illustration of the two-camera tracking system employed in the experiments. The are of interest is shaded, (b) Manual tip position tracking from the two camera video frames. The IMGX and IMGY respectively denote the videos from X and Y directions shown in Figure 8a. IMGX shows task-space origin (BX), base, and mid tip positions marked with the cursor (cross-hair) hovering on top of the distal tip position. IMGY shows the manual estimation of the sections tips from the marker band orientations. This manual setup is simple, effective, and produce good (error $\pm 0.0056 \mathrm{~m}$ ) results. The base section tip band is shown enlarged for clarity.

\subsection{Error Metrics and Results Validation}

The dynamic simulations are carried out in the joint-space for the input pressure profiles associated with the experiments. As noted in Section 4.3, The joint-space trajectories data are recorded at 30 times per second from the simulations. The resulting joint space trajectories are then transformed to task-space trajectories of each section tip $\left(\Psi_{1}, \Psi_{2}\right.$, and $\left.\Psi_{3}\right)$ using the prototype arm kinematic model given by Equation (57) for all $N=1,2,3$ with an additional homogeneous rotational transformation of $-5^{\circ}$ at the base to align with the task-space trajectory tracking camera setup. Note that this transformation is possible because the prototype satisfies the circular arc assumption (assumption AS1 Section 5).

Due to the redundancy of multisection prototype continuum arm, it is important to establish the uniqueness of comparing arm poses. For a given end effector position there are multiple solutions for the shape of the continuum arm (3 point constraints vs. 9DoF). However all the section tip positions of the prototype arm provide a 9 point constraint, i.e., $3 \times 3$ position coordinates. In other words, model is validated against a unique continuum arm pose without redundancy conflicts. This approach can also be thought as a 4 point (including the arm base) shape estimation of the entire arm and conforms to the shape accuracy measuring requirements. Hence, the three uniformly distributed section tip positions (marked with bright red bands in Figure 5a denoting $\Psi_{1}^{e}, \Psi_{2}^{e}$, and $\Psi_{3}^{e}$ ) of the prototype arm are compared against the corresponding section tip positions (annotated in Figure $5 \mathrm{~b}$ as $\Psi_{1}, \Psi_{2}$, and $\Psi_{3}$ ) of the dynamic model.

To quantify the agreement between the experimental and simulation data, instantaneous position errors $\left(\operatorname{ERR}_{i}=\left\|\Psi_{i}^{e}(t)-\Psi_{i}(t)\right\| \forall i \in\{1,2,3\}\right.$ where $i$ is the section number) are computed at each section tip and plotted along with task-space Cartesian coordinates. Finally, in order to quantify the overall model accuracy, maximum of the mean cumulative error of the arm and maximum section error are computed for each experiment. Maximum cumulative error is defined as $\max \left(\sum_{i=1}^{3} \mathrm{ERR}_{i}\right)$ and maximum section error is given by $\max \left(\left[\max \left(\mathrm{ERR}_{1}\right), \max \left(\mathrm{ERR}_{3}\right), \max \left(\mathrm{ERR}_{3}\right)\right]\right)$.

\subsection{Planar Dynamics: In Plane Motion of All Sections}

In the first experiment, the planar dynamic motion is compared of all three sections actuated in order from distal to the base sections against the proposed model. By actuating continuum sections separately, both passive and active responses of the arm can be clearly compared. The following PMA's, denoted by $l_{33}, l_{22}$, and $l_{11}$, are provided with 6 bars, 5 bars, and 5 bars pressure step inputs at $t=0 s, t=3.2 s$, and $t=7.55 \mathrm{~s}$ respectively and maintained throughout the experiment.

As described in Section 5. These pressure commands result all the sections to bend in the $y=0$ plane. The dynamic model is also given the same pressure command sequence and the tip coordinate values are compared in Figure 9. Each subplot denotes the tip coordinates of base (section 1, $\Psi_{1}^{e}$ ), mid (section $2, \Psi_{2}^{e}$ ), and distal (section $3, \Psi_{3}^{e}$ ) continuum sections of the prototype arm respectively along 

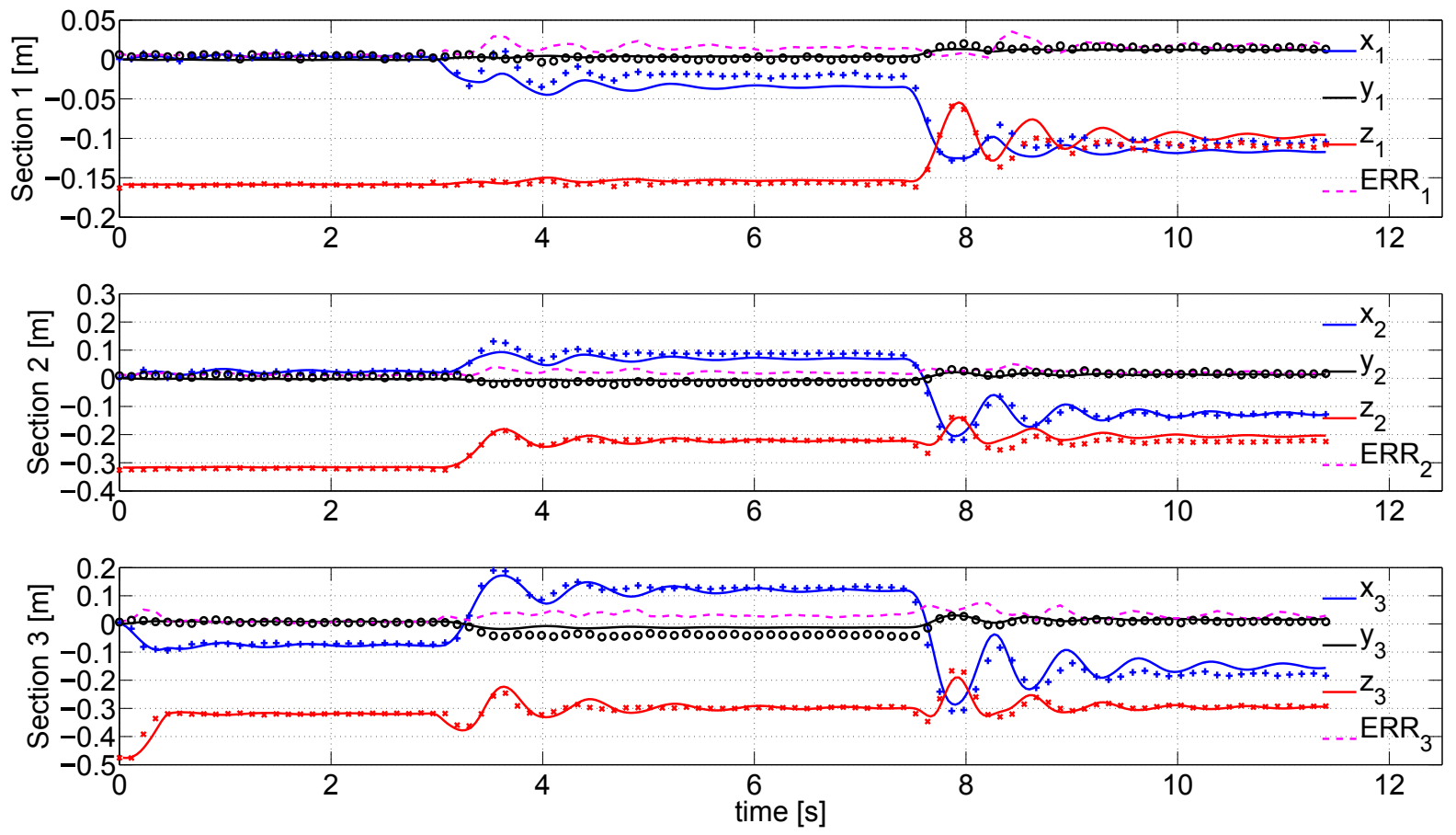

Figure 9: Sequential planar bending of all the sections. Comparison of the dynamic section tip coordinate evolution (solid line) vs. the corresponding on coordinate values of the prototype arm $(\mathrm{X}, \mathrm{Y}, \mathrm{Z}$ experimental data are denoted by $\circ,+, \times$ marks respectively). Position errors, $\operatorname{ERR}_{i}=\left\|\Psi_{i}^{e}-\Psi_{i}\right\| \forall i \in\{1,2,3\}$ are denoted by dashed line in each subplot.
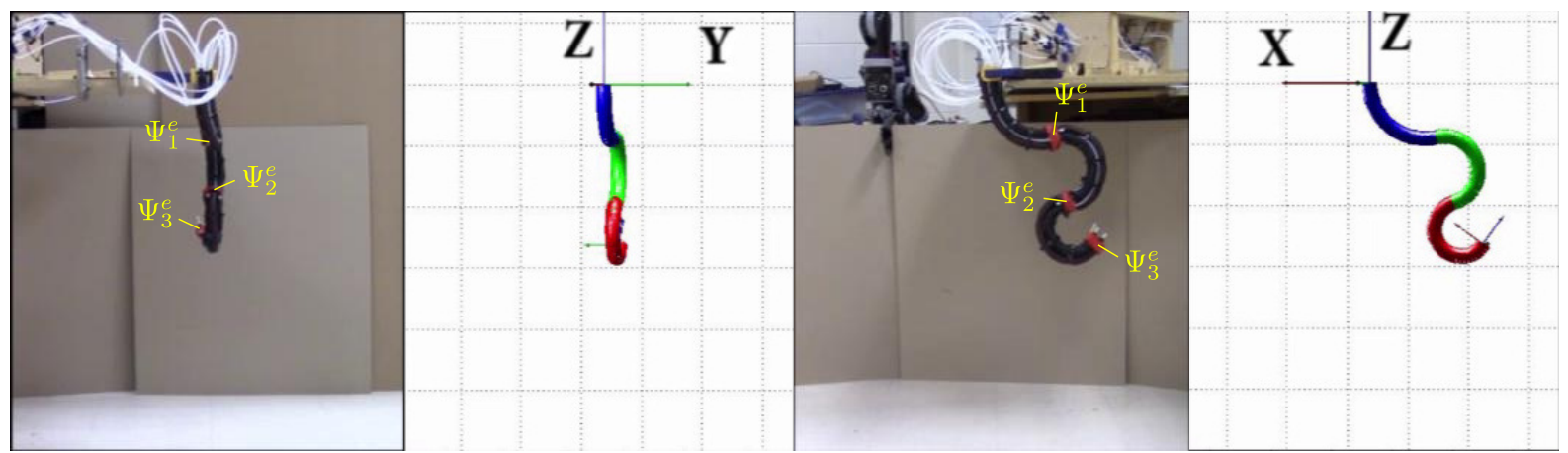

Figure 10: Final pose comparison of the dynamic model and prototype continuum arm for in plane bending of all sections. The real-time side by side comparison is shown in Extension 2. The grid lines of the simulation are spaced at $0.1 \mathrm{~m}$.

with the relevant section tip position errors. The corresponding visual comparison of prototype and simulation model poses at $t=11.4 \mathrm{~s}$ is shown in Figure 10.

From the comparison of output data and error evolution, it can be seen that the proposed model agrees the experimental data well. The maximum section error was $0.0696 \mathrm{~m}$ (recorded at distal section) and the maximum mean error of the arm was $0.0384 \mathrm{~m}$. Noticeable deviations indicated by the errors are observed during step inputs which quickly decay down to steady state values. Closer inspection reveals that the steady state error of $\Psi_{1}^{e}$ begins at the mid section actuation. Further there are some oscillation phase mismatch observed during the transients following base section actuation (after 8sec) with $\Psi_{1}^{e}$ and $\Psi_{3}^{e}$. These deviations can be attributed to the approximated damping coefficients and unmodeled friction/hysteretic effects. The visual real-time side by side comparison is shown in Extension 2.

\subsection{Spatial Dynamics 1: Out of Plane Motion of Two Sections}

Modeling and experimental validation of the out of plane dynamic motion have been two of the key challenges faced by variable length multisection continuum dynamic models and prototype arms. This is due to possible torsional deformations of the arm that cause deviations from the circular arc shape bending deformation (assumption AS1 in Section 2.3). In order to demonstrate the accuracy of the 

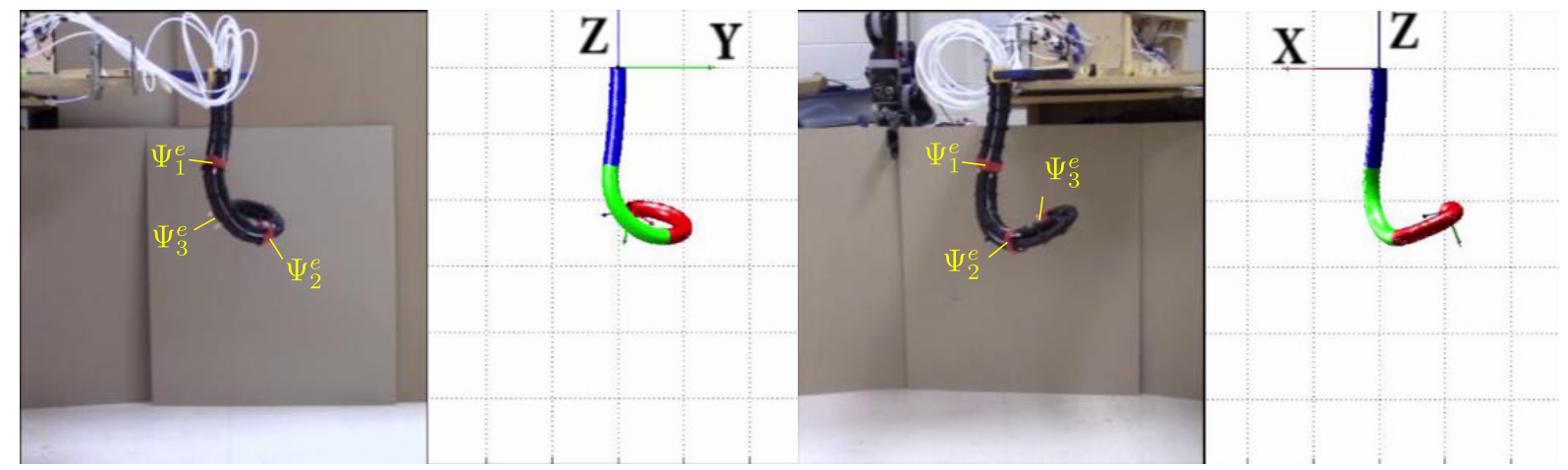

Figure 11: Comparison of the simulated model output against the prototype arm for the out of plane bending of mid and distal sections. Extension 3 shows the real-time side by side comparison. The grid lines of the simulation are spaced at $0.1 \mathrm{~m}$.

proposed model and the efficacy of the prototype arm in mitigating torsional moments, in the second experiment, the actuation of the distal and mid sections are considered in two different bending planes. The base section is kept unactuated to exhibit the passive effects of the gravity induced forces affecting the base section. Step pressure inputs of 5bars and 3 bars were given to $l_{23}$ at $t=0 \mathrm{~s}$ and $l_{33}$ at $t=3.3 \mathrm{~s}$ respectively and maintained throughout. This causes the arm to bend in the task space as shown in Figure 11. It can be seen that the base continuum section's passive bend to balance the forces induced by the mid and distal sections. This phenomenon is correctly modeled by the dynamic model.

As result of the constrainer-enforced torsional rigidity, the arm was able to maintain the assumed bending deformation without deviating from the circular arc shape to produce correct results. This fact can be verified by comparing the relevant coordinate values of experimental data, simulation data during the simulation, and the joint position errors plotted in Figure 12. Particularly the values derived for $\mathbf{D}$ successfully attributes the principle components of the decaying damping. The errors in this experiment also varies during the step input transient stages but settles down quickly. This experiment recorded lower steady state and transient errors than the planar experiment and the possible cause is the unactuated base section.

The maximum section error and mean error of the arm were computed as $0.0539 \mathrm{~m}$ (recorded at distal section) and $0.0363 \mathrm{~m}$ respectively. Note though that, as the base section is unactuated, it can be seen bending slightly under the resulting moments/forces of the actuation of mid and distal sections. This behavior is accurately reflected by the proposed dynamic model (refer to Figures 11 and 12) thus validating the correctness of the model. Extension 3 presents the visual real-time side by side comparison.

\subsection{Spatial Dynamics 2: Out of Plane Motion of All Sections}

In the final experiment, all three sections of the continuum arm are actuated to bend in different bending planes to assume a complex task-space shape shown in Figure 13. Pressure step inputs of 5bars, 3bars, and 3 bars are respectively provided to actuators $l_{33}$ at $t=0 \mathrm{~s}, l_{23}$ at $t=2.55 \mathrm{~s}$ and $l_{11}$ at $t=5.05 \mathrm{~s}$ and maintained during the experiment. The sequence of pressure step inputs cause the arm sections to suddenly bend in different planes. These motions result and propagate passive compliant and dynamic effects on other continuum sections as depicted in Extension 4.

The corresponding tip coordinate trajectories and position errors are compared in Figure 14. This experiments resulted a $0.0671 \mathrm{~m}$ maximum section error (recorded at distal section) and a $0.0410 \mathrm{~m}$ cumulative mean error. According to the results, the proposed modal dynamic model output agrees well with the experimental outcome. Similar to previous experiments, higher position errors can be observed during transient stages following the step inputs but the decaying oscillatory motions and steady state shapes are correctly accounted for by the dynamic model. Noticeable steady state errors can be seen during mid section actuation $(3 \mathrm{~s}-5 \mathrm{~s})$ in the $\mathrm{Z}$ coordinates of $\Psi_{2}^{e}, \Psi_{3}^{e}$. Also during base section actuation $(5.5 \mathrm{~s}-7 \mathrm{~s})$, the $\mathrm{X}$ coordinates of $\Psi_{2}^{e}, \Psi_{3}^{e}$ and $\mathrm{Z}$ coordinate of $\Psi_{1}^{e}$ register some steady state errors. The residual steady state errors can be due to the currently unmodeled hysteresis/friction forces in PMA's. Note that, increasing errors towards the arm tip can be observed in all simulations due to error propagation because of the serial arrangement of continuum sections. By comparing the results of all three experiments, it can be seen that the proposed modal dynamics model for variable length multisection continuum arms successfully predicts and models the dynamic behaviors of the prototype 

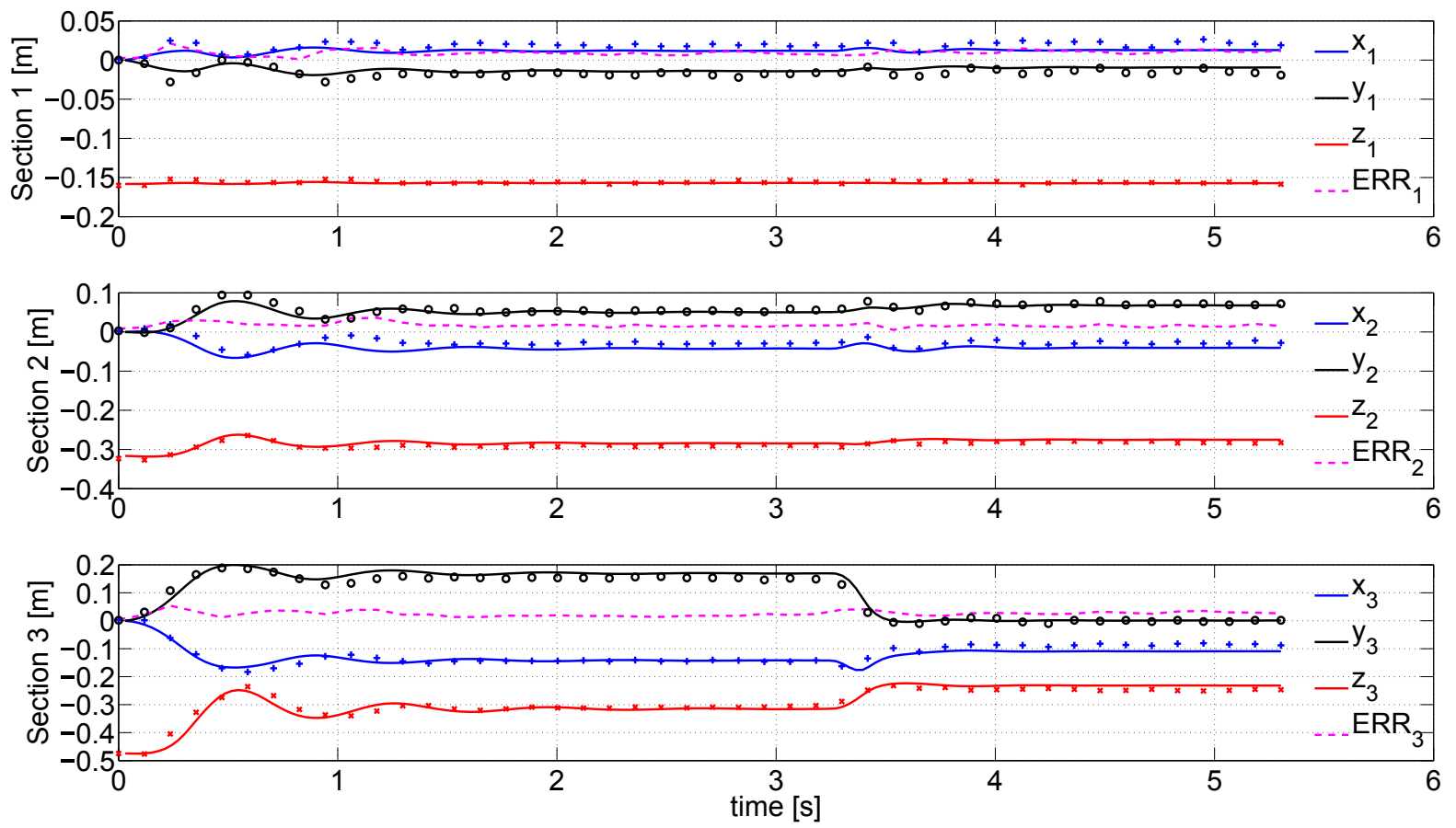

Figure 12: Comparison of the dynamic model coordinates values against the prototype arm section coordinate values (X,Y,Z experimental data are denoted by $\circ,+, \times$ marks respectively) during the out of plane spatial bending of distal and mid sections. The position errors are denoted by dashed lines in each subplot. The final resulting pose is shown in Figure 11.

continuum arm.

\section{Discussion}

\subsection{Numerical Stability and Accuracy of the Proposed Modal Approach}

In this section, the limitations of the curve parametric dynamics and how the proposed modal dynamics circumvents those issues are quantitatively presented.

The curve parametric spatial forward kinematics derived in the joint-space correctly and efficiently model the physical structure of the variable length continuum arms but suffer from numerical instabilities (Jones and Walker, 2007). This instability stems from the circular arc parameters $\left(\lambda_{i}\right.$ and $\theta_{i}$ in Appendix A.1) that becomes undefined for straight arm poses. Therefore, any model that uses this parameterization inherits this instability. The modal kinematics (Godage et al., 2011c, 2015) solve this numerical problems by substituting HTM elements with polynomial MSF's.
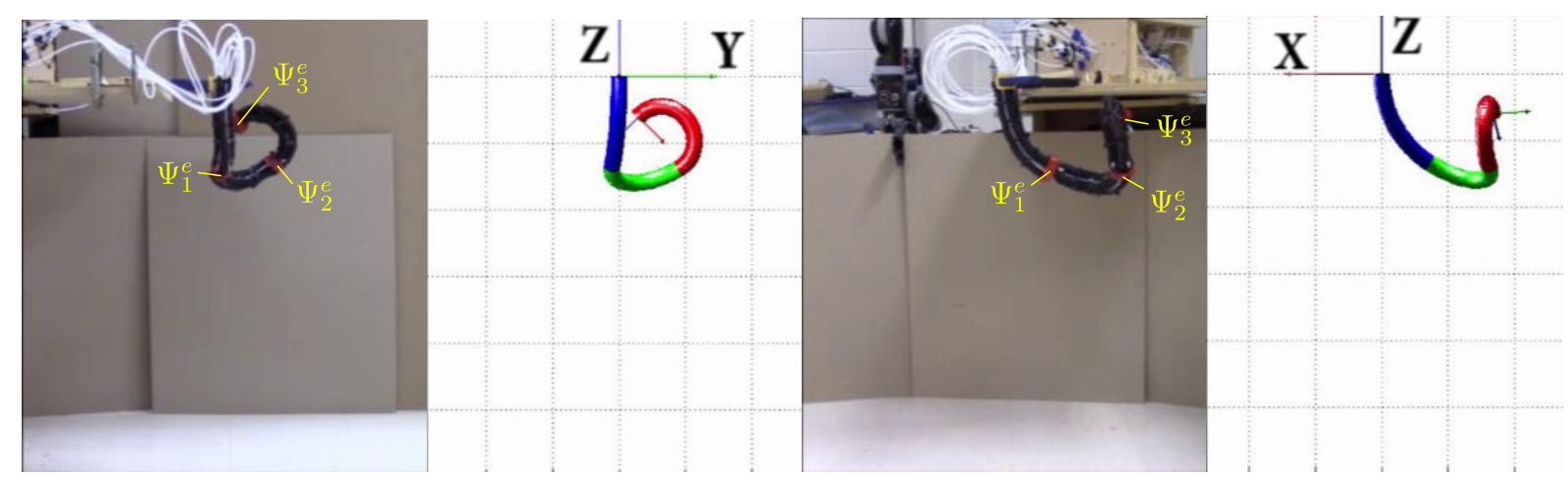

Figure 13: Final pose comparison of the simulated dynamic model output against the prototype multisection arm for the bending of distal, mid, and base sections in different bending planes respectively. Extension 4 presents the real-time side by side comparison. The grid lines of the simulation are spaced at $0.1 \mathrm{~m}$. 

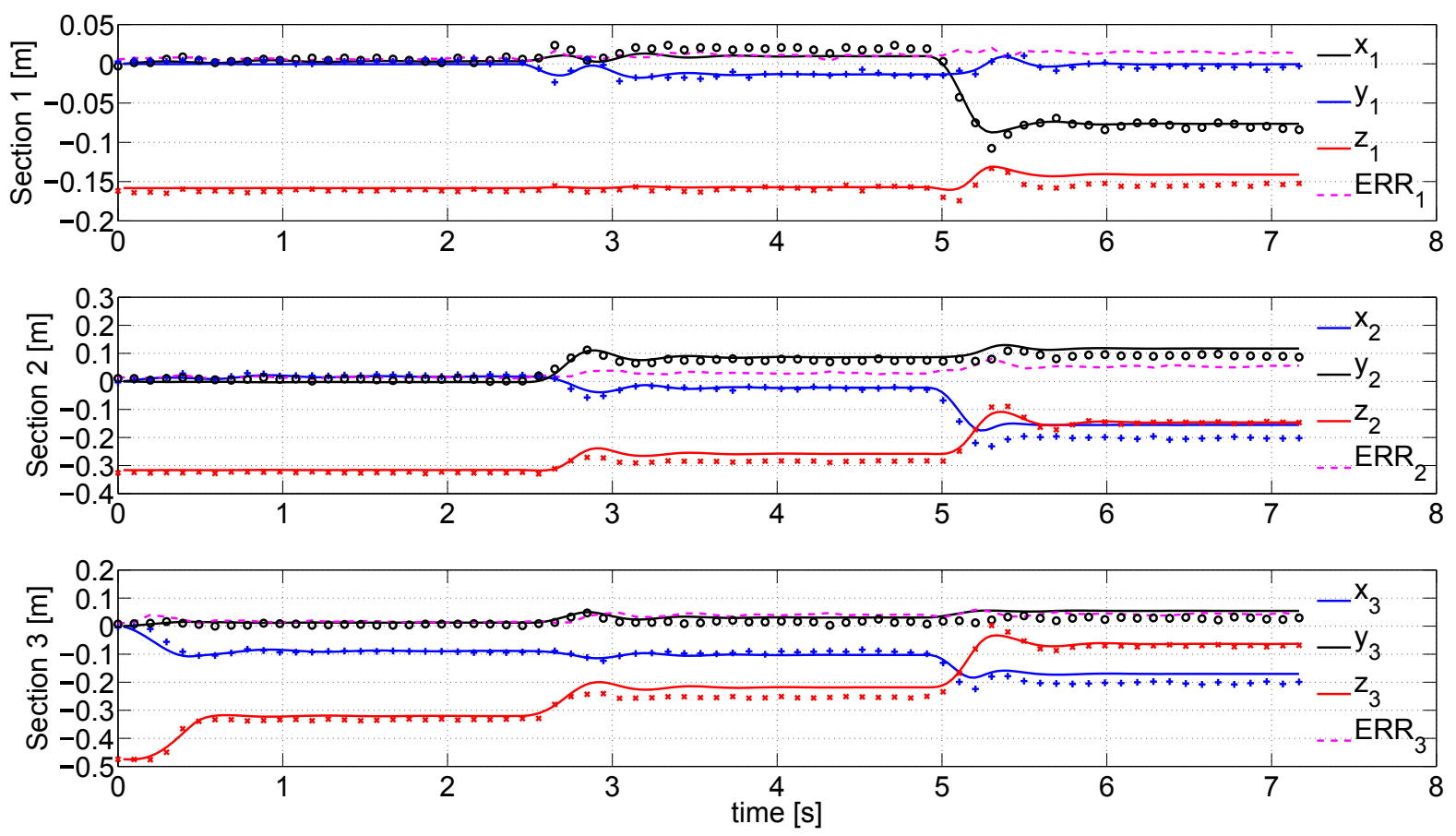

Figure 14: Comparison of the simulated dynamic model section joint coordinate trajectories and errors (denoted by dashed lines) against the prototype arm section coordinate values $(X, Y, Z$ experimental data are denoted by $\circ,+, \times$ marks respectively) during the bending of distal, mid and base sections. Each continuum section bends in differing planes that results in a complex resulting pose shown in Figure 13.

The order of Taylor series expansion defines the fidelity of the MSF's. The order 11 modal approximation (Appendix A.2) results less than 1\% normalized (to prototype length) position error at the arm tip. The accuracy of velocity modal kinematics is illustrated by using the base continuum section parameters. The maximum joint-space velocity for any PMA length change is $0.135 \mathrm{~ms}^{-1}$ (corresponds to $12.5 \mathrm{bar} / \mathrm{sec}$ pressure build up). Given the ranges $\boldsymbol{q}_{1} \in[0,0.065]$ and $\dot{\boldsymbol{q}}_{1} \in[-0.135,0.135]$, the maximum linear velocity error at the tip is $0.0235 \mathrm{~ms}^{-1}$ (range normalized error ${ }^{2} 1.52 \%$ ) when $\boldsymbol{q}_{1}=0.065$ and $\dot{\boldsymbol{q}}_{1}=[0.135,-0.135,-0.135]^{T}$. The maximum angular velocity error at the tip is $0.8238 \mathrm{rads}^{-1}$ (range normalized error $5.7 \%$ ) when $\boldsymbol{q}_{1}=[0.065,0,0.065]^{T}$ and $\dot{\boldsymbol{q}}_{1}=[0.135,-0.135,0.135]^{T}$. These errors were considered acceptable as the curve parametric Jacobians are unreliable with significant errors within singular configurations.

The numerical instabilities of curve parametric kinematics become more significant when extended to formulate dynamics. Therefore, the works such as (Tatlicioglu et al., 2007a,b; Rone and Ben-Tzvi, 2014) assume non-straight starting poses to avoid this numerical problem. To demonstrate the severity of the problem in a numerical example, define the curve parametric kinematics (HTM given in Appendix A.1) based generalized inertia matrix from the linear $\mathrm{KE}$ for the base continuum section $\mathbf{M}_{1 c} \in \mathbb{R}^{3 \times 3}$. For ease of comparison, a single element of $\mathbf{M}_{1 c}$ is used for generating the results. Because there is only one section, from the definition given in Equation (51) define $\left[\mathbf{M}_{1 c}^{v}\right]_{11} \in \mathbb{R}$ as

$$
\left[\mathbf{M}_{1 c}^{v}\right]_{11}=m_{1} \int_{\xi_{1}}\left(\mathbf{J}_{1 c}^{v}\right)_{1}^{T}\left(\mathbf{J}_{1 c}^{v}\right)_{1}
$$

where $\mathbf{J}_{1 c}^{v}=\left(\nabla_{\boldsymbol{q}_{1}} \boldsymbol{p}_{1}^{c}\right) \in \mathbb{R}^{3 \times 3}$ and $\boldsymbol{p}_{1}^{c} \in \mathbb{R}^{3}$ is the curve parametric position vector of $\mathbf{T}_{1}^{c}$.

The integral was symbolically precomputed using MAPLE (2010). Figure 15a shows the rangenormalized percentage error within the range of joint space vectors $l_{12} \in[0,0.065]$ and $l_{13} \in[0,0.065]$ while $l_{11}=0.0325$. Notice the singularity induced errors around $l_{12}=l_{13}=0.0325$. Due to the complex nonlinear terms and accumulative nature of multiplying errors, this produces prohibitively large errors $\left(>10^{3} \%\right)$ within a considerably larger singularity neighborhood. Note that this example is for a single continuum section. On top of the added complexity of nonlinear terms and additive nature of errors, thus it can be deduced that a multisection continuum arm will have considerably larger errors.

\footnotetext{
${ }^{2}$ Range normalized error of $x$ against $x_{a}$ is defined as $\frac{\left\|x-x_{a}\right\|}{\max \left(x_{a}\right)-\min \left(x_{a}\right)}$ where $x_{a}$ is the actual value.
} 


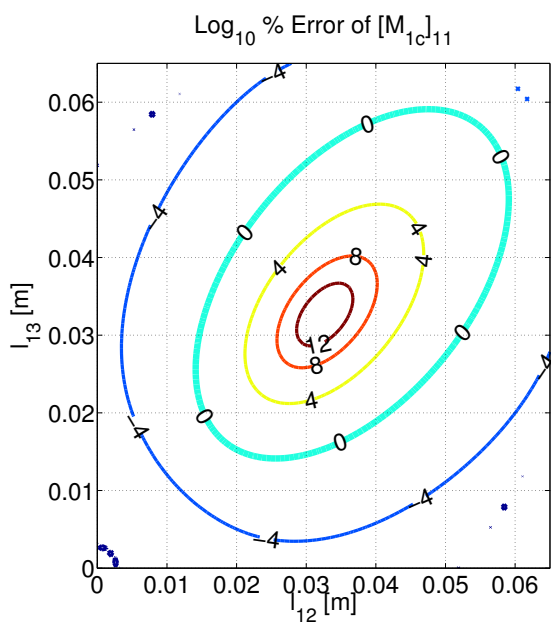

(a)

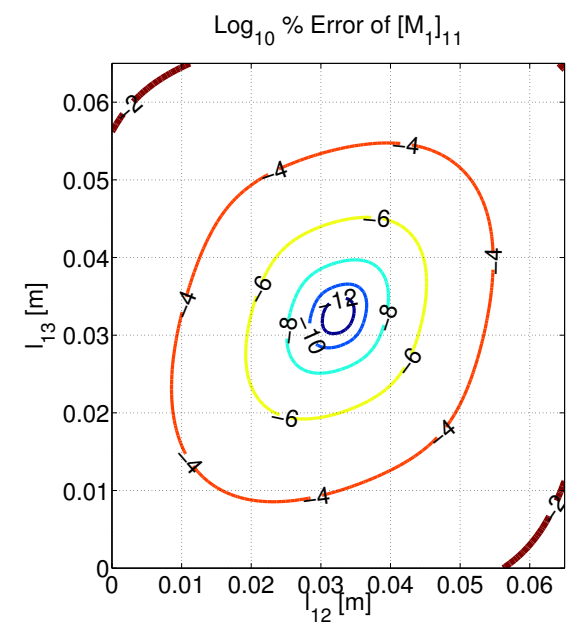

(b)

Figure 15: Generalized inertia matrix numerical error comparison of the base continuum section. (a) $\left[\mathbf{M}_{1 c}^{v}\right]_{11}$. Note the large errors $\left(\geq 10^{3} \%\right.$ ) towards the singularity at $\boldsymbol{q}_{1}=0.0325$ and its (expanded to around $\left\{l_{12}, l_{13}\right\} \in[0.02,0.05]$ ) neighborhood. (b) $\left[\mathbf{M}_{1}^{v}\right]_{11}$. In contrast to Figure $15 \mathrm{a}$, the error is negligible $(\leq 0.014 \%)$ within the entire actuation region. During both simulations $l_{11}=0.0325$.

The numerical stability of the modal dynamics can be clearly shown as follows. Define the modal kinematics (MHTM given in Appendix A.2) based generalized inertia matrix from the linear KE for the base continuum section $\mathbf{M}_{1} \in \mathbb{R}^{3 \times 3}$. From the Equation (51), define $\left[\mathbf{M}_{1}^{v}\right]_{11} \in \mathbb{R}$, which is the modal implementation of $\left[\mathbf{M}_{1 c}^{v}\right]_{11}$, as

$$
\left[\mathbf{M}_{1}\right]_{11}=m_{1} \int_{\xi_{1}}\left(\mathbf{J}_{1}^{v}\right)_{1}^{T}\left(\mathbf{J}_{1}^{v}\right)_{1}
$$

where $\mathbf{J}_{1}^{v}=\left(\nabla_{\boldsymbol{q}_{1}} \boldsymbol{p}_{1}\right) \in \mathbb{R}^{3 \times 3}$ and $\boldsymbol{p}_{1} \in \mathbb{R}^{3}$ is the modal position vector of $\mathbf{T}_{1}$.

Figure 15b shows the range-normalized percentage error (maximum percentage error $\sim 0.02 \%$ ) within the range of joint space vectors $l_{12} \in[0,0.065]$ and $l_{13} \in[0,0.065]$ while $l_{11}=0.0325$. Note that the error $\rightarrow 0$ towards the singularity at $\boldsymbol{q}_{1}=0.0325$. Therefore, the proposed modal dynamics produces excellent results with negligible errors.

\subsection{Computational Efficiency}

The MSF based approach is essentially a high dimensional approximation for HTM elements and naturally, computational efficiency is of concern. However, the modal approach is more computationally efficient than the curve parametric methods and the recursive scheme proposed in this paper improves the computational efficiency. This is achieved by only computing the terms corresponding to the continuum section being evaluated while utilizing the results of previous steps. Figure 16 plots the normalized computing time of MHTM (given by Equation (1)) vs. the multivariate Taylor series expansion order showing that the higher orders (higher accuracy of MSF's) have negligible effect on the computational time. This is due to the structure of MSF's which constituted of increasing powers of the same algebraic terms (see Appendix A.2), that are computationally more efficient than trigonometric evaluations. This has a profound effect when it comes to computing dynamics due to the sheer amount of computational terms that adds up to significant time saving.

An exemplary comparison was carried out on computational efficiency between curve parametric and modal dynamics. Table 3 summarizes the normalized computational time for $\left[\mathbf{M}_{1}^{v}\right]_{11}$ and $\left[\mathbf{M}_{1 c}^{v}\right]_{11}$ in both numerical integration and precomputed integration approach. Numerical integration was carried out utilizing the MATLAB's "integral" routine. Assuming these margins remain the same, then numerical computation time ratio of entire $\mathbf{M}_{1}^{v}$ and $\mathbf{M}_{1 c}^{v}$ will be similar to Table 3 . Thus the proposed MSF based 


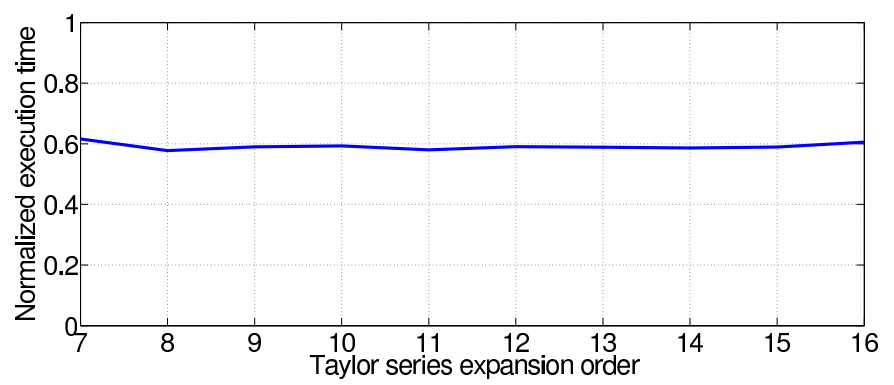

Figure 16: Computation time comparison of MHTM order versus curve parametric HTM. The execution time is normalized to the execution time of $\mathbf{T}_{1}^{c}(25.2 \mu \mathrm{s})$. The mean execution time was calculated by averaging the time for 1000 computations for random $\boldsymbol{q}_{1}$.

Table 3: Computation times of precomputed and numerical integration on curve parametric and modal dynamics. These times are computed for $\left[\mathbf{M}_{1 c}^{v}\right]_{11},\left[\mathbf{M}_{1}^{v}\right]_{11}$ and normalized to the execution time of integral precomputed modal dynamics.

\begin{tabular}{|c|c|c|}
\cline { 2 - 3 } & $\begin{array}{c}\text { Curve parametric dynamics } \\
\text { from Equation (59) }\end{array}$ & $\begin{array}{c}\text { Modal dynamics } \\
\text { from Equation (60) }\end{array}$ \\
\hline \hline Numerical integration & $190-240$ & $110-140$ \\
\hline Precomputed integration & $25-35$ & 1 \\
\hline
\end{tabular}

precomputed integral dynamics formulation proves superior to curve parametric or numerical integration approaches.

Figure 17 shows the normalized time taken to evaluate a single step of the EoM against the number of continuum sections. The algorithm is of $O\left(n^{3}\right)$ complexity, but translates to milliseconds in realtime computations. For instance, the $11^{\text {th }}$ order modal implementation presented herein has a absolute step calculation time around 3ms ( $\sim 20$ when normalized to the execution time of $\left.\mathbf{T}_{1}^{c}, 25.2 \mu s\right)$ for the prototype model. Also, depending on the required accuracy, the order can be further reduced thus improving the computational time. With the MATLAB's ODE15s solver typical simulations spanning 10sec complete around $\sim 10 \mathrm{~s}$ making it suitable for rapid spatial dynamic simulations. The timings of computations are carried out on a AMD A10-4600 quad-core (2.6GHz) laptop with 8Gb RAM. The computational time can be further improved by clever programming and the use of a compiled programming language.

\section{Conclusions}

A novel spatial dynamic model was presented for arbitrarily long, variable length multisection continuum arms. The model solves a longstanding spatial dynamics research problem for this class of continuum

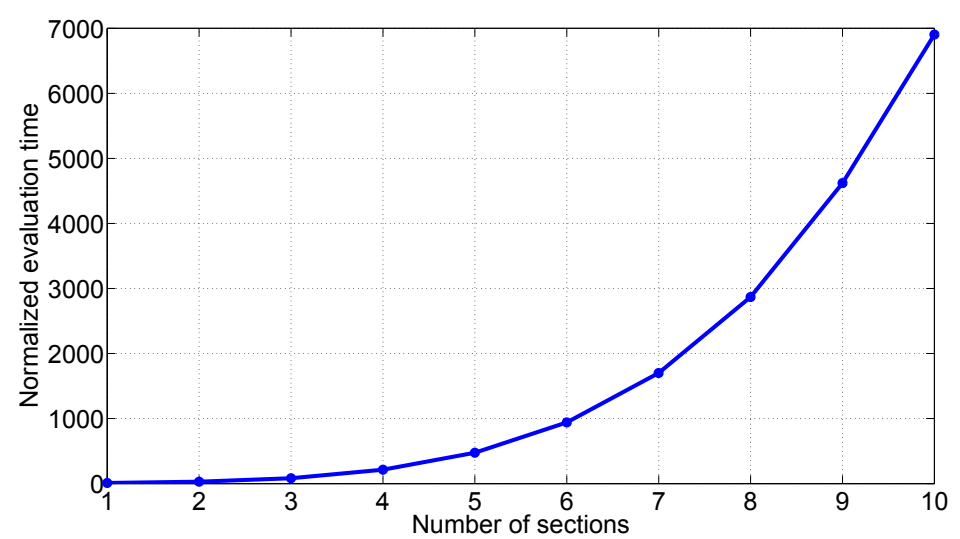

Figure 17: Computational time for one step of the dynamic equation vs. the number of continuum sections of multisection continuum arms. The execution time is normalized to the execution time of $\mathbf{T}_{1}^{c}(25.2 \mu s)$. The algorithm is of $O\left(n^{3}\right)$ complexity but shows very fast times for practical 3-section continuum arms. 
robotic arms through an efficient, highly scalable, recursive computational scheme to yield fast simulation results. The derivation is based on the modal kinematics previously proposed by the authors and hence avoid complex derivations and numerical instabilities associated with curve parametric modeling methods. As a result, the proposed model is structurally accurate, developed in the joint-space, and thus eliminates morphological mappings, and provides better insight to physical operation. Spatial dynamic simulations of the prototype arm successfully attested the numerical stability of the model at singular, straight arm poses. The scalability and numerical efficiency of the proposed modal recursive dynamics were demonstrated by spatial dynamic simulation of a 10-section (30 DoF) continuum arm. Dynamic simulation results were then compared using a PMA actuated variable length prototype continuum arm dynamics for three different experiments including a planar and spatial task-space motions. The results show good agreement overall and the dynamic model was able to correctly simulate the passive and active dynamics of the compliant multisection prototype continuum arm. A discussion on the accuracy and computational efficiency of the proposed model was also presented. To the best of author's knowledge, this is the first structurally accurate spatial dynamic model presented for variable length multisection continuum arms.

\section{Acknowledgments}

This work was in part supported by the European Commission in the ICT-FET OCTOPUS Integrating Project, under contract n. 231608-999596447.

Authors would like to thank Ian Walker of Clemson University for his helpful and insightful feedback.

\section{References}

F. Bertails. Linear time super-helices. In Computer graphics forum, volume 28, pages 417-426. Wiley Online Library, 2009.

W. J. Book. Modeling, design, and control of flexible manipulator arms: A tutorial review. In Procs. of IEEE Conf. on Decision and Control, pages 500-506, 1990.

D. G. Caldwell, G. A. Medrano-Cerda, and M. Goodwin. Control of pneumatic muscle actuators. IEEE Control Systems Magazine, 15(1):40-48, 1995. ISSN 0272-1708.

D. B. Camarillo, C. R. Carlson, and J. K. Salisbury. Configuration tracking for continuum manipulators with coupled tendon drive. Robotics, IEEE Transactions on, 25(4):798-808, 2009.

G. S. Chirikjian and J. W. Burdick. A modal approach to hyper-redundant manipulator kinematics. IEEE Trans. on Robotics and Automation, 10(3):343-354, Jun 1994. ISSN 1042-296X. doi: 10.1109/ 70.294209 .

M. Cianchetti, A. Arienti, M. Follador, B. Mazzolai, P. Dario, and C. Laschi. Design concept and validation of a robotic arm inspired by the octopus. Materials Science and Engineering: C, 31(6): 1230-1239, 2011.

R. Cieslak and A. Morecki. Elephant trunk type elastic manipulator-a tool for bulk and liquid materials transportation. Robotica, 17(01):11-16, 1999.

P. E. Dupont, J. Lock, B. Itkowitz, and E. Butler. Design and control of concentric-tube robots. IEEE Tran. on Robotics, 26(2):209-225, 2010.

R. Featherstone. Rigid body dynamics algorithms, volume 49. Springer Berlin:, 2008.

N. Giri and I. D. Walker. Three module lumped element model of a continuum arm section. In IEEE/RSJ Int. Conf. on Intelligent Robots and Systems, pages 4060-4065, 2011. doi: 10.1109/IROS.2011.6094909.

I. S. Godage, D. T. Branson, E. Guglielmino, G. A. Medrano-Cerda, and D. G. Caldwell. Dynamics for biomimetic continuum arms: A modal approach. In IEEE Int. Conf. on Robotics and Biomimetics, pages 104-109, 2011a.

I. S. Godage, D. T. Branson, E. Guglielmino, G. A. Medrano-Cerda, and D. G. Caldwell. Shape functionbased kinematics and dynamics for variable length continuum robotic arms. In IEEE Int. Conf. on Robotics and Automation, pages 452-457, 2011b. doi: 10.1109/ICRA.2011.5979607. 
I. S. Godage, E. Guglielmino, D. T. Branson, G. A. Medrano-Cerda, and D. G. Caldwell. Novel modal approach for kinematics of multisection continuum arms. In IEEE/RSJ Int. Conf. on Intelligent Robots and Systems, pages 1093-1098, 2011c. doi: 10.1109/IROS.2011.6048086.

I. S. Godage, D. T. Branson, E. Guglielmino, and D. G. Caldwell. Path planning for multisection continuum arms. In IEEE Int. Conf. on Mechatronics and Automation, pages 1208-1213, 2012a. doi: 10.1109/ICMA.2012.6283423.

I. S. Godage, D. T. Branson, E. Guglielmino, and D. G. Caldwell. Pneumatic muscle actuated continuum arms: Modelling and experimental assessment. In IEEE Int. Conf. on Robotics and Automation, pages 4980-4985, 2012b. doi: 10.1109/ICRA.2012.6224949.

Isuru S Godage, Gustavo A Medrano-Cerda, David T Branson, Emanuele Guglielmino, and Darwin G Caldwell. Modal kinematics for multisection continuum arms. Bioinspiration \& biomimetics, 10(3): $035002,2015$.

R.nE. Goldman, A. Bajo, and N. Simaan. Compliant motion control for continuum robots with intrinsic actuation sensing. In IEEE Int. Conf. on Robotics and Automation, pages 1126-1132, may 2011. doi: 10.1109/ICRA.2011.5980000.

I. A. Gravagne and I. D. Walker. On the kinematics of remotely-actuated continuum robots. In IEEE Int. Conf. on Robotics and Automation, pages 2544-2550, 2000. doi: 10.1109/ROBOT.2000.846411.

M. D. Grissom, V. Chitrakaran, D. Dienno, M. Csencits, M. Pritts, B. Jones, W. McMahan, D. Dawson, C. Rahn, and I. D. Walker. Design and experimental testing of the octarm soft robot manipulator. In Defense and Security Symposium, pages 62301F-62301F. Int. Society for Optics and Photonics, 2006.

A. Grzesiak, R. Becker, and A. Verl. The bionic handling assistant: a success story of additive manufacturing. Assembly Automation, 31(4):329-333, 2011.

M. W. Hannan and I. D. Walker. Kinematics and the implementation of an elephant's trunk manipulator and other continuum style robots. Journal of Robotic Systems, 20(2):45-63, 2003.

J. M. Hollerbach. A recursive lagrangian formulation of maniputator dynamics and a comparative study of dynamics formulation complexity. IEEE Tran. on Systems, Man and Cybernetics, 10(11):730-736, Nov 1980. ISSN 0018-9472. doi: 10.1109/TSMC.1980.4308393.

G. Immega, K. Antonelli, K.S. Inc, and BC Vancouver. The ksi tentacle manipulator. In IEEE Int. Conf. on Robotics and Automation, pages 3149-3154, 1995.

B. A. Jones and I. D. Walker. Kinematics for multisection continuum robots. IEEE Trans. on Robotics, 22(1):43-55, 2006a. ISSN 1552-3098. doi: 10.1109/TRO.2005.861458.

B. A. Jones and I. D. Walker. Practical kinematics for real-time implementation of continuum robots. IEEE Transactions on Robotics, 22(6):1087-1099, 2006b.

B. A. Jones and I. D. Walker. Limiting-case analysis of continuum trunk kinematics. In IEEE Int. Conf. on Robotics and Automation, pages 1363-1368, 2007. doi: 10.1109/ROBOT.2007.363174.

B. A. Jones, R. L. Gray, and K. Turlapati. Three dimensional statics for continuum robotics. In IEEE/RSJ Int. Conf. on Intelligent Robots and Systems, pages 2659-2664. IEEE, 2009.

W. Khalil, G. Gallot, and F. Boyer. Dynamic modeling and simulation of a 3-d serial eel-like robot. IEEE Trans. on Systems, Man, and Cybernetics, Part C: Applications and Reviews, 37(6):1259-1268, Nov 2007. ISSN 1094-6977. doi: 10.1109/TSMCC.2007.905831.

J. Li and J. Xiao. Determining "grasping" configurations for a spatial continuum manipulator. In IEEE/RSJ Int. Conf. on Robots and Systems, pages 4207-4214, 2011.

J. Lock, G. Laing, M. Mahvash, and P. E. Dupont. Quasistatic modeling of concentric tube robots with external loads. In IEEE/RSJ Int. Conf. on Intelligent Robots and Systems, pages 2325-2332, 2010.

L. A. Lyons, R. J. Webster, and R. Alterovitz. Motion planning for active cannulas. In IEEE/RSJ Int. Conf. on Intelligent Robots and Systems, pages 801-806, 2009. doi: 10.1109/IROS.2009.5354249. 
T. Mahl, A. Hildebrandt, and O. Sawodny. A variable curvature continuum kinematics for kinematic control of the bionic handling assistant, 2014. ISSN 1552-3098.

MAPLE. Maple User Manual. 2010. ISBN 1-897310-89-7.

W. McMahan and I. D. Walker. Octopus-inspired grasp-synergies for continuum manipulators. In IEEE Int. Conf. on Robotics and Biomimetics, pages 945-950, Bangkok, 2008. doi: 10.1109/ROBIO.2009. 4913126.

W. McMahan, B. A. Jones, I. D. Walker, V. Chitrakaran, A. Seshadri, and D. M. Dawson. Robotic manipulators inspired by cephalopod limbs. In Proceedings CDEN Design Conference, Montreal, Canada, pages 1-10. Citeseer, 2004.

W. McMahan, B. A. Jones, and I. D. Walker. Design and implementation of a multi-section continuum robot: Air-octor. In IEEE/RSJ Int. Conf. on Intelligent Robots and Systems, pages 2578-2585, 2005. doi: 10.1109/IROS.2005.1545487.

W. McMahan, V. Chitrakaran, M. A. Csencsits, D. M. Dawson, I. D. Walker, B. A. Jones, M. B. Pritts, D. Dienno, M. Grissom, and C. D. Rahn. Field trials and testing of the octarm continuum manipulator. In IEEE Int. Conf. on Robotics and Automation, pages 2336-2341, 2006. ISBN 0780395050.

J. S. Mehling, M. A. Diftler, M. Chu, and M. Valvo. A minimally invasive tendril robot for in-space inspection. In IEEE/RAS-EMBS Int. Conf. on Biomedical Robotics and Biomechatronics, pages 690$695,2006$.

H. Mochiyama. Hyper-flexible robotic manipulators. In IEEE Int. Symp. on Micro-NanoMechatronics and Human Science, pages 41-46. IEEE, 2006. ISBN 0780394828.

S. Neppalli, B. Jones, W. McMahan, V. Chitrakaran, I. Walker, M. Pritts, M. Csencsits, C. Rahn, and M. Grissom. Octarm - a soft robotic manipulator. In IEEE/RSJ International Conference on Intelligent Robots and Systems, page 2569, 29 2007-nov. 2 2007. doi: 10.1109/IROS.2007.4399146.

R. S. Penning, J. Jung, J. A. Borgstadt, N. J. Ferrier, and M. R. Zinn. Towards closed loop control of a continuum robotic manipulator for medical applications. In IEEE Int. Conf. on Robotics and Automation, pages 4822-4827, 2011.

F. Renda, M. Giorelli, M. Calisti, and M. Cianchetti. Dynamic model of a multibending soft robot arm driven by cables, 2014. ISSN 1552-3098.

W. S. Rone and P. Ben-Tzvi. Continuum robot dynamics utilizing the principle of virtual power. IEEE Tran. on Robotics, 30(1):275-287, Feb 2014. ISSN 1552-3098. doi: 10.1109/TRO.2013.2281564.

D. C. Rucker and R. J. Webster. Computing jacobians and compliance matrices for externally loaded continuum robots. In IEEE Int. Conf. on Robotics and Automation, pages 945-950. IEEE, 2011 a.

D. C. Rucker and R. J. Webster. Statics and dynamics of continuum robots with general tendon routing and external loading. IEEE Tran. on Robotics, 27(6):1033-1044, 2011b.

A. A. Shabana. Dynamics of multibody systems. Cambridge University Press, 2005. ISBN 0521850118.

N. Simaan, R. Taylor, and P. Flint. A dexterous system for laryngeal surgery. In IEEE Int. Conf. on Robotics and Automation, pages 351-357, 2004. doi: 10.1109/ROBOT.2004.1307175.

H. Takanobu, T. Tandai, and H. Miura. Multi-dof flexible robot base on tongue. In IEEE Int. Conf. on Robotics and Automation, pages 2673-2678, 2004. doi: 10.1109/ROBOT.2004.1307464.

E. Tatlicioglu, I. D. Walker, and D. M. Dawson. Dynamic modelling for planar extensible continuum robot manipulators. In IEEE Int. Conf. on Robotics and Automation, pages 1357-1362, 2007a. ISBN 1424406013.

E. Tatlicioglu, I. D. Walker, and D. M. Dawson. New dynamic models for planar extensible continuum robot manipulators. In IEEE/RSJ Int. Conf. on Intelligent Robots and Systems, pages 1485-1490, 2007b. doi: 10.1109/IROS.2007.4399334. 
D. Trivedi, A. Lotfi, and C. D. Rahn. Geometrically exact models for soft robotic manipulators. IEEE Tran. on Robotics, 24(4):773-780, 2008a.

D. Trivedi, C. D. Rahn, W. M. Kier, and I. D. Walker. Soft robotics: Biological inspiration, state of the art, and future research. Applied Bionics and Biomechanics, 5(3):99-117, 2008b. ISSN 1176-2322.

I. D. Walker, D. M. Dawson, T. Flash, F. W. Grasso, R. T. Hanlon, B. Hochner, W. M. Kier, C. C. Pagano, C. D. Rahn, and Q. M. Zhang. Continuum robot arms inspired by cephalopods. In Procs. of the Int. Society for Optical Engineering, pages 303-314, 2005. ISBN 0819457892.

I. D. Walker, C. Carreras, R. McDonnell, and G. Grimes. Extension versus bending for continuum robots. International Journal of Advanced Robotic Systems, 3(2):1729-8806, 2006.

R. J. Webster, J. M. Romano, and N. J. Cowan. Mechanics of precurved-tube continuum robots. IEEE Tran. on Robotics, 25(1):67-78, feb. 2009. doi: 10.1109/TRO.2008.2006868.

A. Wolf, H. B. Brown, R. Casciola, A. Costa, M. Schwerin, E. Shamas, and H. Choset. A mobile hyper redundant mechanism for search and rescue tasks. In IEEE/RSJ Int. Conf. on Intelligent Robots and Systems, pages 2889-2895 vol.3, 2003. doi: 10.1109/IROS.2003.1249309.

J. Xiao and R. Vatcha. Real-time adaptive motion planning for a continuum manipulator. In IEEE/RSJ Int. Conf. on Intelligent Robots and Systems, pages 5919-5926, 2010. doi: 10.1109/IROS.2010.5648888.

K. Xu and N. Simaan. Analytic formulation for kinematics, statics, and shape restoration of multibackbone continuum robots via elliptic integrals. Journal of Mechanisms and Robotics, 2(1):011006, 2010 .

T. Zheng, D. T. Branson, R. Kang, M. Cianchetti, E. Guglielmino, M. Follador, G. A. Medrano-Cerda, I. S. Godage, and D. G. Caldwell. Dynamic continuum arm model for use with underwater robotic manipulators inspired by octopus vulgaris. In IEEE Int. Conf. on Robotics and Automation, pages 5289-5294, 2012. doi: 10.1109/ICRA.2012.6224685.

\section{Appendix A Homogeneous Transformation Matrices}

\section{A.1 Curve Parametric Homogeneous Transformation Matrix}

Upon actuation, a variable length continuum section deforms in a circular arc (straight section pose is modeled a circular arc of zero curvature). This circular arc shape of any $i^{\text {th }}$ continuum section can be described by three geometrical parameters that are functions of $\boldsymbol{q}_{i}$ : radius of curvature $\lambda_{i}$, angle subtended by the bending arc $\phi_{i}$, and angle of the bending plane with respect to the $+\boldsymbol{X}$ axis $\theta_{i}$ (Figure 3a). Therefore, the curve parametric HTM for any $i^{\text {th }}$ continuum section with respect to its base coordinate frame, $\left\{O_{i}\right\}$ is given by

$$
\begin{aligned}
\mathbf{T}_{i}^{c}\left(\xi_{i}, \boldsymbol{q}_{i}\right) & =\mathbf{R}_{z}\left(\theta_{i}\right) \mathbf{P}_{x}\left(\lambda_{i}\right) \mathbf{R}_{y}\left(\xi_{i} \phi_{i}\right) \mathbf{P}_{x}\left(-\lambda_{i}\right) \mathbf{R}_{z}^{T}\left(\theta_{i}\right) \\
& =\left[\begin{array}{cc}
\mathbf{R}_{i}^{c}\left(\xi_{i}, \boldsymbol{q}_{i}\right) & \boldsymbol{p}_{i}^{c}\left(\xi_{i}, \boldsymbol{q}_{i}\right) \\
\mathbf{0} & 1
\end{array}\right]
\end{aligned}
$$

where $\mathbf{R}_{z} \in \mathbb{R}^{4 \times 4}$ and $\mathbf{R}_{y} \in \mathbb{R}^{4 \times 4}$ are the homogeneous rotational matrices about the $Z$ and $Y$ axes. $\mathbf{P}_{x} \in \mathbb{R}^{4 \times 4}$ is the homogeneous translation matrix along the $X$ axis. $\mathbf{R}_{i}^{3} \in S O(3)$ and $\boldsymbol{p}_{i}^{c} \in \mathbb{R}^{3}$ are the rotational and translation components of the curve parametric HTM. The HTM elements of Equation (A.1) is given below.

$$
\begin{array}{ll}
{\left[\mathbf{R}_{i}^{c}\right]_{11}=\cos ^{2} \theta_{i} \cos \left(\xi_{i} \phi_{i}\right)+\sin ^{2} \theta_{i}} & {\left[\mathbf{R}_{i}^{c}\right]_{12}=\sin \theta_{i} \cos \theta_{i}\left\{\cos \left(\xi_{i} \phi_{i}\right)-1\right\}} \\
{\left[\mathbf{R}_{i}^{c}\right]_{13}=\cos \theta_{i} \sin \left(\xi_{i} \phi_{i}\right)} & {\left[\mathbf{R}_{i}^{c}\right]_{21}=\left[\mathbf{R}_{i}^{c}\right]_{12}} \\
{\left[\mathbf{R}_{i}^{c}\right]_{22}=\sin ^{2} \theta_{i} \cos \left(\xi_{i} \phi_{i}\right)+\cos ^{2} \theta_{i}} & {\left[\mathbf{R}_{i}^{c}\right]_{23}=\sin \theta_{i} \sin \left(\xi_{i} \phi_{i}\right)} \\
{\left[\mathbf{R}_{i}^{c}\right]_{3_{1}}=-\left[\mathbf{R}_{i}^{c}\right]_{13}} & {\left[\mathbf{R}_{i}^{c}\right]_{32}=-\left[\mathbf{R}_{1}^{c}\right]_{23}} \\
{\left[\mathbf{R}_{i}^{c}\right]_{33}=\cos \left(\xi_{i} \phi_{i}\right)} & {\left[\boldsymbol{p}_{i}^{c}\right]_{1}=\lambda_{i} \cos \theta_{i}\left\{1-\cos \left(\xi_{i} \phi_{i}\right)\right\}} \\
{\left[\boldsymbol{p}_{i}^{c}\right]_{2}=\lambda_{i} \sin \theta_{i}\left\{1-\cos \left(\xi_{i} \phi_{i}\right)\right\}} & {\left[\boldsymbol{p}_{i}^{c}\right]_{3}=\lambda_{i} \sin \left(\xi_{i} \phi_{i}\right)}
\end{array}
$$


where $\lambda_{i}=\lambda\left(\boldsymbol{q}_{i}\right), \phi_{i}=\phi\left(\boldsymbol{q}_{i}\right), \theta_{i}=\theta\left(\boldsymbol{q}_{i}\right)$ and

$$
\begin{aligned}
\lambda\left(\boldsymbol{q}_{i}\right) & =\frac{\left(3 L_{i 0}+l_{i 1}+l_{i 2}+l_{i 3}\right) r_{i}}{2 \sqrt{l_{i 1}^{2}+l_{i 2}^{2}+l_{i 3}^{2}-l_{i 1} l_{i 2}-l_{i 1} l_{i 3}-l_{i 2} l_{i 3}}} \\
\phi\left(\boldsymbol{q}_{i}\right) & =\frac{2 \sqrt{l_{i 1}^{2}+l_{i 2}^{2}+l_{i 3}^{2}-l_{i 1} l_{i 2}-l_{i 1} l_{i 3}-l_{i 2} l_{i 3}}}{3 r_{i}} \\
\theta\left(\boldsymbol{q}_{i}\right) & =\arctan \left\{\frac{\sqrt{3}\left(l_{i 3}-l_{i 2}\right)}{l_{i 2}+l_{i 3}-2 l_{i 1}}\right\}
\end{aligned}
$$

\section{A.2 Modal Homogeneous Transformation Matrix}

The MSF's for the MHTM (given by Equation (1)) elements are derived by applying the multivariate Taylor series expansion on the HTM elements (listed in Appendix A.1) at $\boldsymbol{q}_{i}=0$. The $11^{\text {th }}$ order (same order used in the dynamic simulations) MHTM is given below as

$$
\mathbf{T}_{i}\left(\xi_{i}, \boldsymbol{q}_{i}\right)=\left[\begin{array}{cc}
\mathbf{R}_{i}\left(\xi_{i}, \boldsymbol{q}_{i}\right) & \boldsymbol{p}_{i}\left(\xi_{i}, \boldsymbol{q}_{i}\right) \\
\mathbf{0} & 1
\end{array}\right]
$$

where

$$
\begin{aligned}
& {\left[\mathbf{R}_{i}\right]_{11}=1-\frac{A_{2}{ }^{2} A_{1}{ }^{4} \xi_{i}{ }^{10}}{837019575 r_{i}{ }^{10}}+\frac{A_{2}{ }^{2} A_{1}{ }^{3} \xi_{i}{ }^{8}}{4133430 r_{i}{ }^{8}}-\frac{A_{2}{ }^{2} A_{1}{ }^{2} \xi_{i}{ }^{6}}{32805 r_{i}{ }^{6}}+\frac{A_{1} A_{2}{ }^{2} \xi_{i}{ }^{4}}{486 r_{i}{ }^{4}}-\frac{A_{2}{ }^{2} \xi_{i}{ }^{2}}{18 r_{i}{ }^{2}}} \\
& {\left[\mathbf{R}_{i}\right]_{12}=\frac{\sqrt{3} A_{2} A_{3} A_{1}{ }^{4} \xi_{i}{ }^{10}}{837019575 r_{i}{ }^{10}}+\frac{\sqrt{3} A_{2} A_{3} A_{1}{ }^{3} \xi_{i}{ }^{8}}{4133430 r_{i}{ }^{8}}-\frac{\sqrt{3} A_{2} A_{3} A_{1}{ }^{2} \xi_{i}{ }^{6}}{32805 r_{i}{ }^{6}}+\frac{\sqrt{3} A_{2} A_{3} A_{1} \xi_{i}{ }^{4}}{486 r_{i}{ }^{4}}-\frac{\sqrt{3} A_{2} A_{3} \xi_{i}{ }^{2}}{18 r_{i}{ }^{2}}} \\
& {\left[\mathbf{R}_{i}\right]_{13}=-\frac{2 A_{2} A_{1}{ }^{4} \xi_{i}{ }^{9}}{55801305 r_{i}{ }^{9}}+\frac{4 A_{2} A_{1}{ }^{3} \xi_{i}{ }^{7}}{688905 r_{i}{ }^{7}}-\frac{2 A_{2} A_{1}{ }^{2} \xi_{i}{ }^{5}}{3645 r_{i}{ }^{5}}+\frac{2 A_{2} A_{1} \xi_{i}{ }^{3}}{81 r_{i}{ }^{3}}-\frac{A_{2} \xi_{i}}{3 r_{i}}} \\
& {\left[\mathbf{R}_{i}\right]_{22}=1-\frac{A_{3}{ }^{2} A_{1}{ }^{4} \xi_{i}{ }^{10}}{279006525 r_{i}{ }^{10}}+\frac{A_{3}{ }^{2} A_{1}{ }^{3} \xi_{i}{ }^{8}}{1377810 r_{i}{ }^{8}}-\frac{A_{3}{ }^{2} A_{1}{ }^{2} \xi_{i}{ }^{6}}{10935 r_{i}{ }^{6}}+\frac{A_{3}{ }^{2} A_{1} \xi_{i}{ }^{4}}{162 r_{i}{ }^{4}}-\frac{A_{3}{ }^{2} \xi_{i}{ }^{2}}{6 r_{i}{ }^{2}}} \\
& {\left[\mathbf{R}_{i}\right]_{23}=-\frac{2 \sqrt{3} A_{3} A_{1}{ }^{4} \xi_{i}{ }^{9}}{55801305 r_{i}{ }^{9}}+\frac{4 \sqrt{3} A_{3} A_{1}{ }^{3} \xi_{i}{ }^{7}}{688905 r_{i}{ }^{7}}-\frac{2 \sqrt{3} A_{3} A_{1}{ }^{2} \xi_{i}{ }^{5}}{3645 r_{i}{ }^{5}}+\frac{2 \sqrt{3} A_{3} A_{1} \xi_{i}{ }^{3}}{81 r_{i}{ }^{3}}-\frac{\sqrt{3} A_{3} \xi_{i}}{3 r_{i}}} \\
& {\left[\mathbf{R}_{i}\right]_{33}=1-\frac{2 \xi_{i}{ }^{2} A_{1}}{9 r_{i}{ }^{2}}+\frac{2 \xi_{i}{ }^{4} A_{1}{ }^{2}}{243 r_{i}{ }^{4}}-\frac{4 \xi_{i}{ }^{6} A_{1}{ }^{3}}{32805 r_{i}{ }^{6}}+\frac{2 \xi_{i}{ }^{8} A_{1}{ }^{4}}{2066715 r_{i}{ }^{8}}-\frac{4 \xi_{i}{ }^{10} A_{1}{ }^{5}}{837019575 r_{i}{ }^{10}}} \\
& {\left[\mathbf{R}_{i}\right]_{21}=\left[\mathbf{R}_{i}\right]_{12}, \quad\left[\mathbf{R}_{i}\right]_{31}=-\left[\mathbf{R}_{i}\right]_{13}, \quad\left[\mathbf{R}_{i}\right]_{32}=-\left[\mathbf{R}_{i}\right]_{23}} \\
& {\left[\boldsymbol{p}_{i}\right]_{1}=-\frac{A_{2} A_{1}{ }^{4} A_{4} \xi_{i}{ }^{10}}{837019575 r_{i}{ }^{9}}+\frac{A_{2} A_{1}{ }^{3} A_{4} \xi_{i}{ }^{8}}{4133430 r_{i}{ }^{7}}-\frac{A_{2} A_{1}{ }^{2} A_{4} \xi_{i}{ }^{6}}{32805 r_{i}{ }^{5}}+\frac{A_{2} A_{1} A_{4} \xi_{i}{ }^{4}}{486 r_{i}{ }^{3}}-\frac{A_{2} A_{4} \xi_{i}{ }^{2}}{18 r_{i}}} \\
& {\left[\boldsymbol{p}_{i}\right]_{2}=-\frac{\sqrt{3} A_{4} A_{3} A_{1}{ }^{4} \xi_{i}{ }^{10}}{837019575 r_{i}{ }^{9}}+\frac{\sqrt{3} A_{4} A_{3} A_{1}{ }^{3} \xi_{i}{ }^{8}}{4133430 r_{i}{ }^{7}}-\frac{\sqrt{3} A_{4} A_{3} A_{1}{ }^{2} \xi_{i}{ }^{6}}{32805 r_{i}{ }^{5}}+\frac{\sqrt{3} A_{4} A_{1} A_{3} \xi_{i}{ }^{4}}{486 r_{i}{ }^{3}}-\frac{\sqrt{3} A_{4} A_{3} \xi_{i}{ }^{2}}{18 r_{i}}} \\
& {\left[\boldsymbol{p}_{i}\right]_{3}=\frac{2 A_{1}{ }^{4} A_{4} \xi_{i}{ }^{9}}{55801305 r_{i}{ }^{8}}-\frac{4 A_{1}{ }^{3} A_{4} \xi_{i}{ }^{7}}{688905 r_{i}{ }^{6}}+\frac{2 A_{1}{ }^{2} A_{4} \xi_{i}{ }^{5}}{3645 r_{i}{ }^{4}}-\frac{2 A_{1} A_{4} \xi_{i}{ }^{3}}{81 r_{i}{ }^{2}}+\frac{A_{4} \xi_{i}}{3}}
\end{aligned}
$$

and $A_{1}=l_{i 1}^{2}+l_{i 2}^{2}+l_{i 3}^{2}-l_{i 1} l_{i 2}-l_{i 1} l_{i 3}-l_{i 2} l_{i 3}, A_{2}=2 l_{i 1}-l_{i 2}-l_{i 3}, A_{3}=l_{i 2}-l_{i 3}, A_{4}=3 L_{i 0}+l_{i 1}+l_{i 2}+l_{i 3}$.

Depending on the error requirements of the user, one can decide the required order. Note the polynomial nature of terms which results in computational efficiency. Further, for singular straight arm configurations where $l_{i 1}=l_{i 2}=l_{i 3}$, all $A_{1}, A_{2}$, and $A_{3}$ becomes 0 producing the correct MHTM.

\section{Appendix B Mathematical Operators}

The definitions of some of the mathematical operators are listed below.

\section{B.1 Operator $\wedge$}

Let $\mathbf{A} \in \mathbb{R}^{3 \times 1}$ and $\mathbf{A}=\left[a_{1}, a_{2}, a_{3}\right]^{T}$. Then 


$$
\mathbf{A}^{\wedge}=\left[\begin{array}{ccc}
0 & -a_{3} & a_{2} \\
a_{3} & 0 & -a_{1} \\
-a_{2} & a_{1} & 0
\end{array}\right]
$$

\section{B.2 Operator $\vee$}

Let the skew symmetric matrix $\mathbf{A}$ be

$$
\mathbf{A}=\left[\begin{array}{ccc}
0 & -a_{3} & a_{2} \\
a_{3} & 0 & -a_{1} \\
-a_{2} & a_{1} & 0
\end{array}\right]
$$

Then $\vee$ is defined as

$$
\mathbf{A}^{\vee}=\left[a_{1}, a_{2}, a_{3}\right]^{T}
$$

\section{Appendix C Recursive Kinematic Relationships}

The complete derivation of recursive kinematic relationships are included below. These lay the foundation for developing the recursive dynamics.

\section{C.1 Position and Orientation}

Expanding the MHTM given by Equation (2) for a multisection continuum arm, consider the position vector and orientation matrix for the $i^{\text {th }}$ continuum section given as

$$
\begin{aligned}
\Theta_{i} & =\mathbf{R}_{1} \mathbf{R}_{2} \ldots \mathbf{R}_{i-1} \mathbf{R}_{i} \\
\Psi_{i} & =\boldsymbol{p}_{1}+\mathbf{R}_{1} \boldsymbol{p}_{2}+\cdots+\mathbf{R}_{1} \mathbf{R}_{2} \ldots \mathbf{R}_{i-1} \boldsymbol{p}_{i}
\end{aligned}
$$

where $\boldsymbol{p}_{k}=p\left(\xi_{k}, \boldsymbol{q}_{k}, r_{k}, L_{0 k}\right), \mathbf{R}_{k}=R\left(\xi_{k}, \boldsymbol{q}_{k}, r_{k}, L_{0 k}\right)$ are the functional implementation of position and rotational matrices. $k$ is the section index. Input of section radius and original length can accommodate mechanically different continuum sections. The dependency variables are dropped for brevity.

The recursive position vector is therefore

$$
\Theta_{i}=\left(\mathbf{R}_{1} \mathbf{R}_{2} \ldots \mathbf{R}_{i-1}\right) \mathbf{R}_{i}=\Theta_{i-1} \mathbf{R}_{i}
$$

where $\Theta_{i-1}=\mathbf{R}_{1} \mathbf{R}_{2} \ldots \mathbf{R}_{i-1}$ from the definition given in Equation (C.1)

Similarly, using the result of Equation (C.3), Equation (C.2) is manipulated to

$$
\begin{aligned}
\Psi_{i} & =\left(\boldsymbol{p}_{1}+\cdots+\mathbf{R}_{1} \ldots \mathbf{R}_{i-2} \boldsymbol{p}_{i-1}\right)+\left(\mathbf{R}_{1} \ldots \mathbf{R}_{i-1}\right) \boldsymbol{p}_{i} \\
& =\Psi_{i-1}+\Theta_{i-1} \boldsymbol{p}_{i}
\end{aligned}
$$

where $\Psi_{i-1}=\boldsymbol{p}_{1}+\mathbf{R}_{1} p_{2}+\cdots+\mathbf{R}_{1} \ldots \mathbf{R}_{i-2} \boldsymbol{p}_{i-1}$ from the definition given by Equation (C.2).

\section{C.2 Angular Velocity Jacobian}

From the angular velocity Jacobian definition given in Equation (18), $\left(\mathbf{J}_{i}^{\omega}\right)_{j} \in \mathbb{R}^{3}$ can be written as

$$
\left(\mathbf{J}_{i}^{\omega}\right)_{j}=\left(\Theta_{i}^{T} \Theta_{i, j}\right)^{\vee}
$$

For ease of notation, considering $\Omega \equiv \omega^{\wedge}$, define $\left(\mathbf{J}_{i}^{\Omega}\right)_{j}=\left(\mathbf{J}_{i}^{\omega}\right)_{j}^{\wedge} \in \mathbb{R}^{3 \times 3}$. Therefore 


$$
\left(\mathbf{J}_{i}^{\Omega}\right)_{j}=\Theta_{i}^{T} \Theta_{i, j}
$$

Note that $\left(\mathbf{J}_{i}^{\Omega}\right)_{j}=0 \forall j>i$ because $\boldsymbol{q}^{i} \mapsto \mathbf{J}_{i}^{\Omega}\left(\boldsymbol{q}^{i}\right)$. Considering $\boldsymbol{q}^{i}=\left[\boldsymbol{q}^{i-1}, \boldsymbol{q}_{i}\right]$, hence depending on whether the partial derivative variable belong to either $q_{j} \in \boldsymbol{q}^{i-1}$ or $q_{j} \in \boldsymbol{q}_{i}$, the result can be simplified to two separate cases. When $q_{j} \in \boldsymbol{q}^{i-1}$, substituting Equation (C.3) into Equation (C.6) gives

$$
\begin{aligned}
\left(\mathbf{J}_{i}^{\Omega}\right)_{j} & =\mathbf{R}_{i}^{T}\left(\Theta_{i-1}^{T} \Theta_{i-1, j}\right) \mathbf{R}_{i} \\
& =\mathbf{R}_{i}^{T}\left(\mathbf{J}_{i-1}^{\Omega}\right)_{j} \mathbf{R}_{i}
\end{aligned}
$$

where, by definition given in Equation (C.6), $\left(\mathbf{J}_{i-1}^{\Omega}\right)_{j}=\Theta_{i-1}^{T} \Theta_{i-1, j}$.

Similarly, when $q_{j} \in \boldsymbol{q}_{i}$, Equation (C.6) becomes

$$
\begin{aligned}
\left(\mathbf{J}_{i}^{\Omega}\right)_{j} & =\mathbf{R}_{i}^{T}\left(\Theta_{i-1}^{T} \Theta_{i-1}\right) \mathbf{R}_{i, j} \\
& =\mathbf{R}_{i}^{T} \mathbf{R}_{i, j}
\end{aligned}
$$

where $\Theta_{i-1}^{T} \Theta_{i-1}=\mathbf{I}_{3}$.

Combining these results gives

$$
\left(\mathbf{J}_{i}^{\Omega}\right)_{j}= \begin{cases}\mathbf{R}_{i}^{T}\left(\mathbf{J}_{i-1}^{\Omega}\right)_{j} \mathbf{R}_{i} & ; q_{j} \in \boldsymbol{q}^{i-1} \\ \mathbf{R}_{i}^{T} \mathbf{R}_{i, j} & ; q_{j} \in \boldsymbol{q}_{i}\end{cases}
$$

\section{C.3 Linear Velocity Jacobian}

From the $\mathbf{J}_{i}^{v}$ definition given in Equation (19), the $\left(\mathbf{J}_{i}^{v}\right)_{j} \in \mathbb{R}^{3}$ is given by

$$
\left(\mathbf{J}_{i}^{v}\right)_{j}=\Theta_{i}^{T} \Psi_{i, j}
$$

Similar to the $\mathbf{J}_{i}^{\Omega}$ recursive derivation, when $q_{j} \in \boldsymbol{q}^{i-1}$, substituting Equation (C.4) into Equation (C.10) and simplifying give

$$
\left(\mathbf{J}_{i}^{v}\right)_{j}=\mathbf{R}_{i}^{T}\left\{\left(\Theta_{i-1}^{T} \Psi_{i-1, j}\right)+\left(\Theta_{i-1}^{T} \Theta_{i-1, j}\right) \boldsymbol{p}_{i}\right\}
$$

Substituting $\left(\mathbf{J}_{i-1}^{v}\right)_{j}=\Theta_{i-1}^{T} \Psi_{i-1, j}$ and $\left(\mathbf{J}_{i-1}^{\Omega}\right)_{j}=\Theta_{i-1}^{T} \Theta_{i-1, j}$ derived from the definitions given by Equations (C.10) and (C.6) into Equation (C.11) yields

$$
\left(\mathbf{J}_{i}^{v}\right)_{j}=\mathbf{R}_{i}^{T}\left\{\left(\mathbf{J}_{i-1}^{v}\right)_{j}+\left(\mathbf{J}_{i-1}^{\Omega}\right)_{j} \boldsymbol{p}_{i}\right\}
$$

When $q_{j} \in \boldsymbol{q}_{i}$, Equation (C.10) becomes

$$
\left(\mathbf{J}_{i}^{v}\right)_{j}=\mathbf{R}_{i}^{T}\left(\Theta_{i-1}^{T} \Theta_{i-1}\right) \boldsymbol{p}_{i, j}=\mathbf{R}_{i}^{T} \boldsymbol{p}_{i, j}
$$

where $\Theta_{i-1}^{T} \Theta_{i-1}=\mathbf{I}_{3}$.

Combining the results of Equations (C.12) and (C.13) gives

$$
\left(\mathbf{J}_{i}^{v}\right)_{j}= \begin{cases}\mathbf{R}_{i}^{T}\left\{\left(\mathbf{J}_{i-1}^{v}\right)_{j}+\left(\mathbf{J}_{i-1}^{\Omega}\right)_{j} \boldsymbol{p}_{i}\right\} & ; q_{j} \in \boldsymbol{q}^{i-1} \\ \mathbf{R}_{i}^{T} \boldsymbol{p}_{i, j} & ; q_{j} \in \boldsymbol{q}_{i}\end{cases}
$$




\section{C.4 Angular Velocity Hessian}

For a $N$ section continuum arm with each section having 3 DoF, define $\mathbf{H}_{i}^{\omega} \in \mathbb{R}^{3 \times 3 N \times 3 N}$ of $\omega_{i}$ as

$$
\mathbf{H}_{i}^{\omega}=\nabla_{\boldsymbol{q}} \mathbf{J}_{i}^{\omega}
$$

From Equation (C.15), define $\left(\mathbf{H}_{i}^{\omega}\right)_{j k} \in \mathbb{R}^{3}$ as

$$
\left(\mathbf{H}_{i}^{\omega}\right)_{j k}=\left(\mathbf{J}_{i}^{\omega}\right)_{j, k}
$$

For the ease of notation, define $\left(\mathbf{H}_{i}^{\Omega}\right)_{j k} \equiv\left(\mathbf{H}_{i}^{\omega}\right)_{j k}^{\wedge} \in \mathbb{R}^{3 \times 3}$. Following the case-wise derivation, when $q_{k} \in \boldsymbol{q}^{i-1}$, substituting Equation (C.9) into Equation (C.16) gives

$$
\left(\mathbf{H}_{i}^{\Omega}\right)_{j k}= \begin{cases}\mathbf{R}_{i}^{T}\left(\mathbf{J}_{i-1}^{\Omega}\right)_{j, k} \mathbf{R}_{i} & ;\left\{q_{j}, q_{k}\right\} \in \boldsymbol{q}^{i-1} \\ 0 & ; q_{j} \in \boldsymbol{q}_{i}, q_{k} \in \boldsymbol{q}^{i-1}\end{cases}
$$

When $q_{k} \in \boldsymbol{q}_{i}$

$$
\left(\mathbf{H}_{i}^{\Omega}\right)_{j k}= \begin{cases}\mathbf{R}_{i, k}^{T}\left(\mathbf{J}_{i-1}^{\Omega}\right)_{j} \mathbf{R}_{i}+\mathbf{R}_{i}^{T}\left(\mathbf{J}_{i-1}^{\Omega}\right)_{j} \mathbf{R}_{i, k} & ; q_{j} \in \boldsymbol{q}^{i-1}, q_{k} \in \boldsymbol{q}_{i} \\ \mathbf{R}_{i, k}^{T} \mathbf{R}_{i, j}+\mathbf{R}_{i}^{T} \mathbf{R}_{i, j, k} & ;\left\{q_{j}, q_{k}\right\} \in \boldsymbol{q}_{i}\end{cases}
$$

Substituting the definition of $\left(\mathbf{H}_{i-1}^{\Omega}\right)_{j k}=\left(\mathbf{J}_{i-1}^{\Omega}\right)_{j, k}$ derived from Equation (C.16) into Equations (C.17), (C.18) and combining the result gives

$$
\left(\mathbf{H}_{i}^{\Omega}\right)_{j k}= \begin{cases}\mathbf{R}_{i}^{T}\left(\mathbf{H}_{i-1}^{\Omega}\right)_{j k} \mathbf{R}_{i} & ;\left\{q_{j}, q_{k}\right\} \in \boldsymbol{q}^{i-1} \\ \mathbf{R}_{i, k}^{T}\left(\mathbf{J}_{i-1}^{\Omega}\right)_{j} \mathbf{R}_{i}+\mathbf{R}_{i}^{T}\left(\mathbf{J}_{i-1}^{\Omega}\right)_{j} \mathbf{R}_{i, k} & ; q_{j} \in \boldsymbol{q}^{i-1}, q_{k} \in \boldsymbol{q}_{i} \\ 0 & ; q_{j} \in \boldsymbol{q}_{i}, q_{k} \in \boldsymbol{q}^{i-1} \\ \mathbf{R}_{i, k}^{T} \mathbf{R}_{i, j}+\mathbf{R}_{i}^{T} \mathbf{R}_{i, j, k} & ;\left\{q_{j}, q_{k}\right\} \in \boldsymbol{q}_{i}\end{cases}
$$

This completes the derivation of $\left(\mathbf{H}_{i}^{\Omega}\right)_{j k}$.

\section{C.5 Linear Velocity Hessian}

For a $N$ section continuum arm with each section having 3 DoF, define the linear velocity Hessian, $\mathbf{H}_{i}^{v} \in \mathbb{R}^{3 \times 3 N \times 3 N}$ as

$$
\mathbf{H}_{i}^{v}=\nabla_{\mathbf{q}} \mathbf{J}_{i}^{v}
$$

From Equation (C.20), define $\left(\mathbf{H}_{i}^{v}\right)_{j k} \in \mathbb{R}^{3 \times 1}$ as

$$
\left(\mathbf{H}_{i}^{v}\right)_{j k}=\left(\mathbf{J}_{i}^{v}\right)_{j, k}
$$

When $q_{k} \in \boldsymbol{q}^{i-1}$, substituting the recursive $\left(\mathbf{J}_{i}^{v}\right)_{j}$ from Equation (C.14) into Equation (C.21) yields

$$
\left(\mathbf{H}_{i}^{v}\right)_{j k}= \begin{cases}\mathbf{R}_{i}^{T}\left\{\left(\mathbf{J}_{i-1}^{v}\right)_{j, k}+\left(\mathbf{J}_{i-1}^{\Omega}\right)_{j, k} \boldsymbol{p}_{i}\right\} & ;\left\{q_{j}, q_{k}\right\} \in \boldsymbol{q}^{i-1} \\ 0 & ; q_{j} \in \boldsymbol{q}_{i}, q_{k} \in \boldsymbol{q}^{i-1}\end{cases}
$$

Similarly, when $q_{k} \in \boldsymbol{q}_{i}$

$$
\left(\mathbf{H}_{i}^{v}\right)_{j k}= \begin{cases}\mathbf{R}_{i, k}^{T}\left\{\left(\mathbf{J}_{i-1}^{v}\right)_{j}+\left(\mathbf{J}_{i-1}^{\Omega}\right)_{j} \boldsymbol{p}_{i}\right\}+\mathbf{R}_{i}^{T}\left(\mathbf{J}_{i-1}^{\Omega}\right)_{j} \boldsymbol{p}_{i, k} & ; q_{j} \in \boldsymbol{q}^{i-1}, q_{k} \in \boldsymbol{q}_{i} \\ \mathbf{R}_{i, k}^{T} \boldsymbol{p}_{i, j}+\mathbf{R}_{i}^{T} \boldsymbol{p}_{i, j, k} & ;\left\{q_{j}, q_{k}\right\} \in \boldsymbol{q}_{i}\end{cases}
$$


Substitute the definition of $\left(\mathbf{H}_{i-1}^{\Omega}\right)_{j k}=\left(\mathbf{J}_{i-1}^{\Omega}\right)_{j, k}$ and $\left(\mathbf{H}_{i-1}^{v}\right)_{j k}=\left(\mathbf{J}_{i-1}^{v}\right)_{j, k}$ derived from Equations (C.16) and (C.21). Combining the results of Equations (C.22) and (C.23) gives

$$
\left(\mathbf{H}_{i}^{v}\right)_{j k}= \begin{cases}\mathbf{R}_{i}^{T}\left\{\left(\mathbf{H}_{i-1}^{v}\right)_{j}+\left(\mathbf{H}_{i-1}^{\Omega}\right)_{j} \boldsymbol{p}_{i}\right\} & ;\left\{q_{j}, q_{k}\right\} \in \boldsymbol{q}^{i-1} \\ \mathbf{R}_{i, k}^{T}\left\{\left(\mathbf{J}_{i-1}^{v}\right)_{j}+\left(\mathbf{J}_{i-1}^{\Omega}\right)_{j} \boldsymbol{p}_{i}\right\}+\mathbf{R}_{i}^{T}\left(\mathbf{J}_{i}^{\Omega}\right)_{j} \boldsymbol{p}_{i, k} & ; q_{j} \in \boldsymbol{q}^{i-1}, q_{k} \in \boldsymbol{q}_{i} \\ 0 & ; q_{j} \in \boldsymbol{q}_{i}, q_{k} \in \boldsymbol{q}^{i-1} \\ \mathbf{R}_{i, k}^{T} \boldsymbol{p}_{i, j}+\mathbf{R}_{i}^{T} \boldsymbol{p}_{i, j, k} & ;\left\{q_{j}, q_{k}\right\} \in \boldsymbol{q}_{i}\end{cases}
$$

This completes the derivation of $\left(\mathbf{H}_{i}^{v}\right)_{j k}$.

\section{Appendix D Algebraic Identities}

For simplification of generalized inertia matrix terms and symbolic precomputation of the integrals, following algebraic identities are used. This results in significant computational efficiency as discussed in Section 6.2.

\section{D.1 Algebraic Identity 1: $\boldsymbol{B}^{T} \operatorname{Diag}(1,1,2) \boldsymbol{B}=\mathbb{T}_{2}\left(\mathbf{A}^{T} \mathbf{A}\right)$}

Proof. Let $\mathbf{A} \in \mathbb{R}^{3 \times 3}$ be a skew symmetric matrix and, $B=\mathbf{A}^{\vee}$, and $[\boldsymbol{B}]_{i}=b_{i}$.

Consider the following result.

$$
\boldsymbol{B}^{T} \operatorname{Diag}(1,1,2) \boldsymbol{B}=b_{1}^{2}+b_{2}^{2}+2 b_{3}^{2}
$$

Now consider

$$
\begin{aligned}
\mathbb{T}_{2}\left(\mathbf{A}^{T} \mathbf{A}\right) & =b_{1}^{2}+b_{2}^{2}+2 b_{3}^{2} \\
& =\boldsymbol{B}^{T} \operatorname{Diag}(1,1,2) \boldsymbol{B}
\end{aligned}
$$

Proof completed.

\section{D.2 Algebraic Identity 2: $\mathbb{T}_{2}\left(\mathbf{A}^{T} \mathbf{H B}\right)=\mathbb{V}^{\mathrm{T}}\left(\widetilde{\mathbf{A}} \widetilde{\mathbf{B}}^{T}\right) \mathbb{V}(\mathbf{H})$}

Proof. Let $[\mathbf{A}]_{i j}=a_{i j},[\mathbf{B}]_{i j}=b_{i j}$, and $\{r, k\} \in\{1,2,3\}$. Then

$$
\begin{aligned}
\mathbb{T}_{2}\left(\mathbf{A}^{T} \mathbf{H B}\right) & =\sum_{s}\left(\sum_{r} a_{s r}\left(\sum_{k} h_{r k} b_{k s}\right)\right), \quad s \in\{1,2\} \\
& =\sum_{s} \sum_{r} \sum_{k}\left(a_{s r} b_{k s}\right) h_{r k} \\
& =\mathbb{V}^{\mathrm{T}}\left(\widetilde{\mathbf{A}} \widetilde{\mathbf{B}}^{T}\right) \mathbb{V}(\mathbf{H})
\end{aligned}
$$

Proof completed.

\section{D.3 Algebraic Identity 3: $\boldsymbol{A}^{T} \mathbf{H} \boldsymbol{B}=\mathbb{V}^{\mathrm{T}}\left(\boldsymbol{A} \boldsymbol{B}^{T}\right) \mathbb{V}(\mathbf{H})$}

Proof. Let $\{\boldsymbol{A}, \mathbf{B}\} \in \mathbb{R}^{3 \times 1}, \mathbf{H} \in \mathbb{R}^{3 \times 3}$, and defined as $[\boldsymbol{A}]_{i}=a_{i}, \quad[\boldsymbol{B}]_{j}=b_{j}, \quad[\mathbf{H}]_{i j}=h_{i j}$. Then

$$
\begin{aligned}
\boldsymbol{A}^{T} \mathbf{H} \boldsymbol{B} & =\sum_{i} a_{i}\left(\sum_{j} h_{i j} b_{j}\right)=\sum_{i} \sum_{j} a_{i} h_{i j} b_{j} \\
& =\sum_{i} \sum_{j}\left(a_{i} b_{j}\right) h_{i j}=\mathbb{V}^{\mathrm{T}}\left(\boldsymbol{A} \boldsymbol{B}^{T}\right) \mathbb{V}(\mathbf{H})
\end{aligned}
$$

Proof completed. 